Journal of Marine Teabnology Vol.I, year 2021

he a dif

$\triangle 0$ NAUTICA 


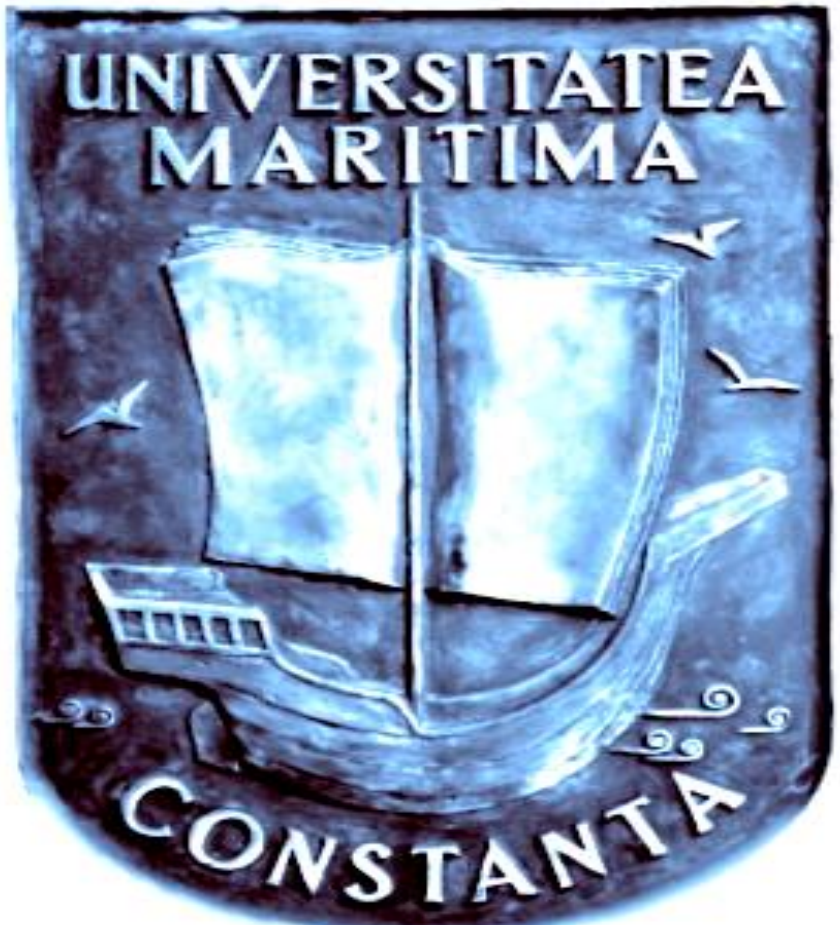


ISSN 1844-6116 
This Journal has been founded in 2008 as a biannual publication of Constanta Maritime University/ROMANIA

\section{TOPICS}

- Marine Science and Engineering

- Marine Environmental Issues

- Marine Renewable Energy and Sustainability

- Maritime Safety

- Marine Chemistry

- Marine Corrosion and Material Science

- Ship Design, Building Technologies

- Ocean Engineering

- Advanced Technologies for MET

- Advances in numerical methods for marine engineering

- Algorithms for multidisciplinary problems in marine engineering

- Other related topics

\section{EDITOR IN CHIEF}

Prof. PhD. Eng. Mariana PANAITESCU (Constanta Maritime University, Constanta, Romania)

\section{VICE EDITOR IN CHIEF}

Assoc. Prof. PhD. Eng. Feiza MEMET (Constanta Maritime University, Constanta, Romania)

\section{EDITORIAL BOARD}

Prof. PhD. Angelica M.BAYLON (Maritime Academy of Asia and the Pacific, Mariveles Bataan, PHILIPPINES) Prof. VAdm Eduardo Ma R SANTOS (Maritime Academy of Asia and the Pacific, Mariveles Bataan, PHILIPPINES) Prof. Dr. Sc. Nataliya Danailova NIKOLOVA (University: Nikola Vaptsarov Naval Academy - Varna, Bulgaria) Assist. Prof. WALTER K. NADOLNY (Marine Transportaton and Environmental Management - State University of New York Maritime College, Bronx, NY, USA)

Assoc. Prof. PhD, MSc/ Dip. Momoko KITADA (WORLD MARITIME UNIVERSITY, Malmö, Sweden)

Prof. PhD. Hu QINYOU (Shanghai Maritime University, Merchant Marine College, Shanghai, CHINA) Assoc. Prof. Mahmoud Reza HAGHDOUSTI (University: Iran Maritime Training Center, Tehran, IRAN) Assoc. Prof. PhD. Kalin Spasov KALINOV (Nikola Vaptsarov Naval Academy, Varna, Bulgaria)

DSc., Professor Irina MAKASHINA (International Education Center of Admiral Ushakov State Maritime University, Novorossiysk, RUSSIA)

Prof. PhD. Igor SMIRNOV (Admiral Ushakov Maritime State University, Navigating Faculty, Novorossiysk, RUSSIA) Prof. PhD. Tomasz NEUMANN (Gdynia Maritime University, Faculty of Navigation, POLAND)

Prof. PhD. Axel LUTTENBERGER (University of Rijeka, Faculty of Maritime Studies Rijeka, CROATIA)

Prof. PhD. Stoianka Georgieva Tania PETKOVA (University "Prof .dr. Assen Zlatarov", Burgas, BULGARIA)

Professor Boyan Kirilov MEDNIKAROV ("Nikola Vaptsarov" Naval Academy, Varna, BULGARIA)

Prof. PhD Eng. Petko Stoyanov PETKOV (University "Prof .dr. Assen Zlatarov", Burgas, BULGARIA)

Prof. Dr. Nil GULER (Istanbul Technical University, Maritime Faculty Tuzla, TURKEY)

Prof. PhD.Eng. Gheorghe - Constantin IONESCU (University from Oradea, Faculty of Civil Engineering and Architecture, Department of Civil Engineering, Oradea, ROMANIA)

Prof. PhD.Eng. Gheorghe LAZAROIU (University POLITEHNICA of Bucharest, Faculty of Power Engineering, Department of Energy Production and Use, Romania)

Prof. PhD.Eng. Cornel PANAIT (Constanta Maritime University, Electromechanical Faculty, Constanta, ROMANIA) Prof. PhD.Eng. Viorel-Fanel PANAITESCU (Constanta Maritime University, Electromechanical Faculty, Constanta, ROMANIA)

Prof. PhD.Eng. Nicolae BUZBUCHI (Constanta Maritime University, Electromechanical Faculty, Constanta, ROMANIA)

Prof .PhD. eng. Dan POPA (Constanta Maritime University, Electromechanical Faculty, Constanta, ROMANIA)

Prof. PhD. Boyan Kirilov MEDNIKAROV (Rector of Nikola Vaptsarov Naval Academy, Varna,Bulgaria) 
Prof. PhD. Marusya LUBCHEVA (Black Sea Institute Burgas, BULGARIA)

PhD. Ricardo Rodriguez - MARTOS DAUER (Departament de Ciencia I Enginyeria Nautiques/ Universitat Politecnica de Catalunya/SPAIN)

Prof. PhD. Sergii RUDENKO (Odessa National Maritime University/ UKRAINE)

\title{
SCIENTIFIC BOARD
}

Assoc. Prof. PhD. Eugen BARSAN (Constanta Maritime University, Constanta, ROMANIA)

Assoc. Prof. PhD.Eng. Dumitru DELEANU (Constanta Maritime University, Constanta, ROMANIA)

Assoc. Prof. PhD.Eng. Liviu-Constantin STAN (Constanta Maritime University, Constanta, ROMANIA)

Prof. PhD. Habil. Razvan TAMAS (Constanta Maritime University, Constanta, ROMANIA)

Assoc. Prof. PhD. Alexandra RAICU (Constanta Maritime University, Constanta, ROMANIA)

Assoc. Prof. PhD. Gabriel RAICU (Constanta Maritime University, Constanta, ROMANIA)

Milen DIMITROV (Black Sea Institute Burgas, BULGARIA)

Assoc Prof. Dr. Docent Eng. Bohoz APRAHAMIAN (Technical University of Varna, BULGARIA)

Senior Lecturer Captain Mahmoud El-Sayed El- BAWAB (Arab Academy for Science Technology and Maritime

Transport, Alexandria, EGYPT )

Lecturer Capt Emre UCAN (Dokuz Eylul University, TURKEY)

Luis G. EVIDENTE (John B. Lacson Colleges Foundation, PHILIPPINES)

Francesc Xavier MARTINEZ DE OSES (Departament de Ciencia i Enginyeria

Nautiques/Universitat Politecnica de Catalunya/SPAIN)

Teresa J.LEO (Universidad Politecnica de Madrid /SPAIN)

Prof. Dr. Valeriu LUNGU Valeriu (Moldova Technical University)

Prof. PhD. Magdalena MITKOVA (University "Prof. d-r Assen Zlatarov" Burgas, BULGARIA)

Assoc. Prof. Svitlana GLOVATSKA (Odessa National Maritime University, UKRAINA)

Assoc. Prof. PhD. Lyubcho LYUBCHEV (University 'Prof. d-r Assen Zlatarov” Burgas, BULGARIA)

Prof. PhD. Irena MARKOVSKA (University 'Prof. d-r Assen Zlatarov” Burgas, BULGARIA)

Tatiana KOVTUN (Odessa National Maritime University, UKRAINA)

Assoc. Prof. Mykola ADAMCHUK (Odessa National Maritime University, UKRAINA)

S.L. Iunusova ELMAZ (Odessa National Maritime University, UKRAINA)

Assist. Prof. PhD.Sabina NEDKOVA (University "Prof. d-r Assen Zlatarov" Burgas, BULGARIA)

Vladimir KANEV (Expert WEB Application Software Sofia, BULGARIA)

Senior Lecturer Vanyio GRANCIAROV (University "Prof. d-r Assen Zlatarov” Burgas, BULGARIA)

Milen DIMITROV (Black Sea Institute Burgas, BULGARIA)

Assist.Prof. PhD. Evghenyi RUDENKO (Odessa National Maritime University, UKRAINA)

\section{EDITORIAL SECRETARY}

Senior lecturer PhD. Eng. VOICU Ionut (Constanta Maritime University, Constanta ROMANIA)

COMPUTERIZED EDITING

IGNAT Irina-Andreea (Constanta Maritime University/ROMANIA)

\section{WEB ADMINISTRATOR}

POPESCU George

\author{
JOURNAL ADDRESS \\ Journal of Marine Technology and Environment \\ Constanta Maritime University, 104, Mircea cel Batran Street, 900663, Constanta, Romania \\ Tel: +40 $241664740 / 107$ \\ Fax: +40 241617260 \\ E-mail: jmte@cmu-edu.eu \\ http://cmu-edu.eu/jmte/
}

EDITURA NAUTICA

Constanta Maritime University

CONSTANTA MARITIME UNIVERSITY, 104, MIRCEA CEL BATRAN STREET, 900663, 


\section{CONTENTS}

APPROACHES BASED ON ARTIFICIAL INTELLIGENCE FOR WATER SUPPLY

1. SYSTEMS

GABRIELA ANDREI ${ }^{1}$

1 Constanta Maritime University, Faculty of Naval Electro-Mechanics, ROMANIA

2. THE APPLICATIONS OF GIS FOR DEVELOPMENT SOME CHOOSING AREAS ANGELICA M. BAYLON ${ }^{1}$, MARIANA PANAITESCU ${ }^{2}$, FANEL-VIOREL PANAITESCU ${ }^{2}$

${ }^{1}$ Maritime Academy of Asia and the Pacific, Mariveles Bataan, PHILIPPINES

2 Constanta Maritime University, Faculty of Naval Electro-Mechanics, ROMANIA

EVALUATION OF THE QUALITY STANDARDS OF THE WATER DISCHARGED

3. INTO THE SEA AFTER WASHING THE EXHAUST GASES

REMUS-CRISTINEL COJOCARU ${ }^{1}$

${ }^{1}$ Constanta Maritime University, Doctoral School of Mechanical Engineering and Mechatronic, ROMANIA

COMPARATIVE CFD BASED PARAMETRIC ANALYSIS OF GAS FLOW IN TWO

4. CONTER-FLOW WET SCRUBBER SYSTEMS

DANIELA-ELENA JUGANARU ${ }^{1}$, MARIANA PANAITESCU ${ }^{1}$, LIVIU-CONSTANTIN STAN $^{1}$

${ }^{1}$ Constanta Maritime University, Faculty of Naval Electro-Mechanics, ROMANIA

5. TOOLS TO ENGAGE YOUTH IN ENVIRONMENTAL ISSUES DURING COVID-19 PANDEMIC: CONSTANTA MARITIME UNIVERSITY APPROACH MIRELA-IULIANA SUNDRI ${ }^{1}$, FEIZA MEMET $^{1}$

${ }^{1}$ Constanta Maritime University, Faculty of Naval Electro-Mechanics, ROMANIA

POINT OF VIEW ON THE PERCEPTION OF FUTURE PROFESSIONALS ON

6. ENERGY EFFICIENCY OF REFIRGERATION SYSTEMS IONELA TICU ${ }^{1}$, ELENA GOGU ${ }^{2}$

${ }^{1}$ Constanta Maritime University, Faculty of Naval Electro-Mechanics, ROMANIA

${ }^{2}$ Gheorghe Duca" Technological High School, Constanta, ROMANIA

7. INSTALLATION OF SUBMARINE CABLES IN THE OFFSHORE WIND INDUSTRY AND THEIR IMPACT ON THE MARINE ENVIRONMENT

VALERIU-FLORIAN VASILESCU ${ }^{1}$, DUMITRU DINU ${ }^{1}$

${ }^{1}$ Constanta Maritime University, Doctoral School of Mechanical Engineering and Mechatronic, Constanta, Romania 



\title{
APPROACHES BASED ON ARTIFICIAL INTELLIGENCE FOR WATER SUPPLY SYSTEMS
}

\author{
Gabriela Andrei ${ }^{1}$ \\ ${ }^{1}$ Constanta Maritime University, Faculty of Naval Electro-Mechanics, 104 Mircea cel Batran Street, 900663, Constanta, \\ Romania, e-mail address:gabriela.andrei@cmu-edu.eu
}

\begin{abstract}
In the current context of the existence of life, of the development of human activities, water has a double importance:

- environmental factor, generator of ecological systems

- "raw material" for certain uses (drinking water, industrial water, fish farming, leisure, etc.)

The current problems in the field of water supply are due to:

- exponential increase of water demand;

- the limited water resources and their uneven distribution, which requires large and expensive works of development and accumulation of water;

- deterioration of the quality of water sources, as a result of human activity and the emergence of industries that discharge waste, containing very stable impurities, difficult to remove from water, in the processes of water purification or treatment;

- increase in standards on quality conditions that must be met by water delivered to the population [1].

For the extensive and intensive development of water supplies, a concrete solution, already existing in the area of Artificial Intelligence is given by heuristic methods and Evolutionary Calculus.

This article provides an overview of the role of the most important metaheuristics, based on evolutionary concepts - Evolutionary computation and behavioral patterns inspired by biology - Swarm calculus) in the case of of water supply systems and their subsystems, with exemplification in the case of a model network (Scheme of the network distribution in Hanoi), taking into account water quality (its treatment with chlorine).
\end{abstract}

Key words: The water supply, artificial intelligence, swarm, swarm of ants.

\section{INTRODUCTION}

Swarm Intelligence is a new computational paradigm based on the study of the behaviors of socially organized groups in the animal world (ant colonies, groups of birds, lots of fish, bee colonies, bacterial colonies).

Collective intelligence is based on the exchange of information between members. The interaction between the neighbors, as well as the interactions with the environment, allows them to build complex structures and achieve their goal (the optimal road to certain locations, avoiding predators, etc.). The success of this type of behavior inspired the development of the two metaheuristics: Particle Swarm Optimization (PSO) and Ant Colony Optimization (ACO).

AMF is part of the category of evolutionary stochastic optimization algorithms and is inspired by the search behavior of an ant colony, to detect the food source and bring it to the mound on the shortest path.
This behavior is based on traces of pheromones secreted and deposited by ants in their movements, traces that serve as an indirect form of communication. Routes with higher pheromone intensity are chosen primarily by members of the colony and thus the pheromone concentration on those routes is continuously amplified. In a very general way, the MFA operates with a group of artificial ants, each of them corresponding to a complete solution of the problem to be solved and having a certain quality, in relation to the intended optimization objective. Based on these individual qualities, the intensities of the pheromone are initialized for the possible option variants, ending a global iteration (a search stage or a time step - admitted here discreetly as opposed to the process in nature). In the next global iteration, a new group of ants is generated stochastically (possible solutions), but more favoring the options with increased pheromone intensity. After evaluating them in relation to the objective of the problem and readjusting the intensity of the pheromone for all options, it is 
Journal of Marine Technology and Environment expected that some of them will become stronger and more attractive to be selected in future iterations. The process ceases after an imposed number of global iterations, and the most successful ant (solution) in the last iteration has the chance to place itself very close to the optimal global solution of the problem [4].

An example of implementing this strategy was developed in 2008 by Liana Ioana VUȚA and Radu POPA, in search of a model for optimal sizing of hydraulic networks, which includes both the annual costs of investing in pipelines and the costs associated with water chlorination. From a hydraulic point of view, the sizing must comply with the minimum pressure restrictions on the calculation flow regime, estimated as the highest for the respective network.

\section{IMPLEMENTATION OF THE ALGORITHM}

In terms of water quality, a frequency chart imposed during the year is allowed, having different classes of flows to consumers (below the calculation flows) and for each class, is determined the concentration of chlorine required at network entry, the amount of chlorine associated and its cost so that the residual chlorine in the network exceeds a specified minimum concentration. As an optimization method, the extended ant algorithm (AMF), described in detail for the hydraulic part in Popa and Vuţă, 2007 [3], was adopted for this extended problem.

For the formulation of the mathematical model, it is taken into account that, as a rule, when dimensioning the water networks, the positions of the consumption nodes and the required calculation flows, the route of the connection pipes and their lengths, as well as the position of the supply tank and the hydraulic load at the tank are known. The problem is as, from a discrete number of standardized values, choosing the appropriate diameter for each pipe so that at any node in the network to ensure at least a minimum value of the working pressure at the calculation mode, but minimizing the cost of making pipes. In any optimization method, this objective is achieved by selecting the lowest standard diameters (having the lowest unit costs) that lead to compliance with the minimum pressure restrictions imposed.

However, the small diameters imply high water velocities on the pipes, reduced retention times in the system, limited loss of chlorine during transport in the network and therefore the need for a lower chlorine concentration when leaving the tank to ensure a minimum level of residual chlorine required at consumers. This happens when operating in calculation flow mode, and at this mode the low cost of pipes that respond to hydraulic pressure restrictions is associated with the lower costs required for chlorination at the
Year 2021, Vol.I

supply tank. It is known that the distribution network, once built, operates only a limited fraction of the time under the conditions of calculation flows. The lower flows of network determine the lower velocities of water on the pipes and the longer transit times in the system. As a result, the loss of chlorine in the network is amplified, and in order to maintain the minimum permitted level of residual chlorine in consumers, an increased concentration (and therefore higher treatment costs) is required when leaving the tank. In order to take into account both aspects of costs, it is proposed that the optimal sizing analysis be done on an annual basis. In this sense, a specified lifetime, $\mathrm{T}$ years, is allowed for the considered network, and the annual cost corresponding to a possible solution, $\phi$, of pipe diameters, Vh $(\varphi)$, will be taken simply, considering two cases, if the solution $\varphi$ complies with the minimum load restriction imposed, respectively, if the restriction is violated.

$$
V_{h}(\varphi)=\left\{\begin{array}{c}
\frac{1}{T} \sum_{i=1}^{I} L_{i} \cdot c_{s i j} \\
\frac{1}{T} \sum_{i=1}^{I} L_{i} \cdot c_{s i j}+p_{h} \cdot \Delta H_{\max }
\end{array}\right.
$$

I este the number of pipes in the network, $\mathrm{Li}$ is the length of the pipe $\mathrm{i}$, cij is the unit cost (per linear meter) of the pipe of standard diameter dij chosen for pipe i, $\Delta$ Hmax represents the maximum pressure deficit in the network compared to the minimum imposed pressure, and ph is a cost penalty factor for violating the minimum service pressure restriction (at calculation flows).

Regarding the mode of the network flows throughout the year, the frequency chart of the requested flows is allowed, presumably the same for all consumers. In the case shown in fig. 1, for instance: during $35 \%$ of the year (i.e. $035 \cdot 8760=3066$ ) it is requested between 50 and $75 \%$ of the design flow $\mathrm{q}$, having an average value per class of $q k=0,625 \cdot q$. Let $K$ be the number of flow classes with the average values per class $\mathrm{qk}$ (in $\mathrm{m}^{3} / \mathrm{h}$ ) and the requesting time $\Delta \mathrm{tk}$ (in $\mathrm{h} /$ year ), for $\mathrm{k}=1,2, \ldots, \mathrm{K}$

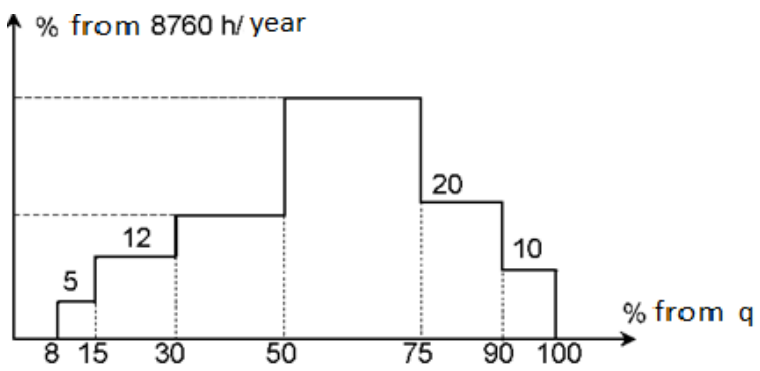

Figure 1 Example of frequency graph (as a fraction

of the annual duration) of consumption flows (as fractions of the calculation flow q) [3]

In the case of each possible design solution, $\varphi$, after calculating the contribution of the pipes to the annual 
Journal of Marine Technology and Environment costs using the relation (1), $\mathrm{K}$ runs of the network hydraulic mode are performed, on the diameters of the solution $\varphi$, but successively taking into account the consumption flows from the nodes with values $\mathrm{qk}, \mathrm{k}=$ $1, \mathrm{~K}$. For each run there is a corresponding flow from the tank, QRk (in $\mathrm{m} 3 / \mathrm{h}$ ) and, through an iterative process, the concentration of chlorine entering the network is determined, CRk (in $\mathrm{mg} / \mathrm{l}$ ), which makes it possible to reach the minimum allowed value, Clim (în $\mathrm{mg} / \mathrm{l}$ ), of the residual chlorine, in the most disadvantaged node of the system. Next, the annual cost for chlorine treatment in the tank for solution $\varphi, \mathrm{V}_{\mathrm{c}}(\varphi)$, is obtained using the relation:

$$
V_{C}(\varphi)=10^{-3} \mathrm{v}_{\mathrm{C}} \frac{1}{\mathrm{~T}} \cdot \sum_{\mathrm{k}=1}^{\mathrm{K}} \mathrm{Q}_{\mathrm{Rk}} \cdot \Delta \mathrm{t}_{\Delta \mathrm{k}} \cdot \mathrm{C}_{\mathrm{R}_{\mathrm{k}}}
$$

where $\mathrm{V}_{\mathrm{c}}$ is the cost implied by producing / handling / injecting one kilogram of chlorine that is able to ensure the necessary concentrations $\mathrm{C}_{\mathrm{Rk}}$ when leaving the tank. The total annual costs for the construction of pipes and for the chlorination of water at the source, in the case of a possible design solution, $\varphi$, is obtained by adding (1) and (2), meaning:

$$
V_{h}(\varphi)=V_{h}(\varphi)+V_{C}(\varphi)
$$

and the performance function targeted in the optimal sizing model is:

$$
\min \left\{\left(V_{h}(\varphi)\right\}\right.
$$

Obviously, for each combination of standard diameters of the various pipes in the network, there are corresponding pipe costs, minimum loads in the network, chlorine concentrations at the source, on the required flow classes and, respectively, different chlorination costs to ensure the compliance with the requirements for water quality.
Year 2021, Vol.I

In order to view the subject matter in the AMF logic framework, to solve it and to provide a qualitative interpretation of the results, the authors based their work on the distribution network of Hanoi, used in the literature, as described in the chart below [2]. The lengths of the 34 pipes in the network and the serial numbers is the usable diameters are shown in table 2 , while table 3 contains the design flows $q$ (in $\mathrm{m} 3 / \mathrm{h}$ ) stressed in the network nodes. The network is allowed flat, with the load at the tank $\mathrm{Hr}=100 \mathrm{~m}$ and the minimum allowable load in nodes of $\mathrm{Hmin}=30 \mathrm{~m}$.

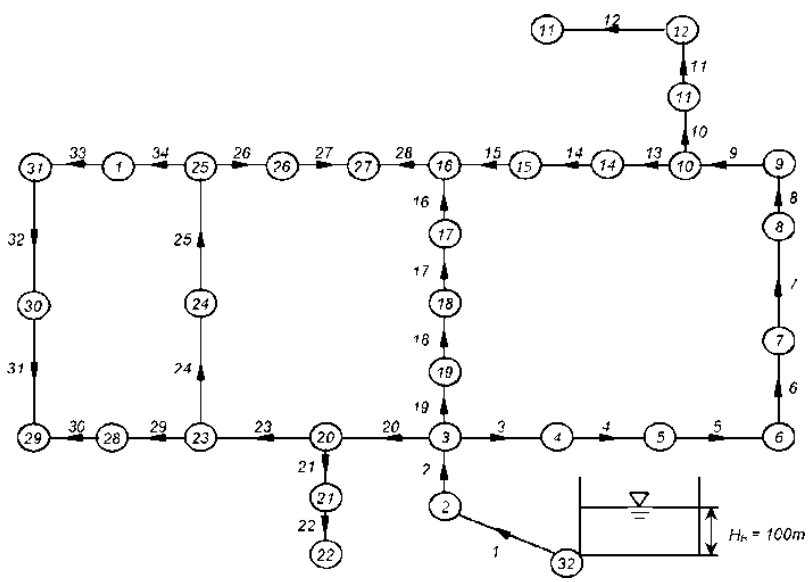

Figure 2 Diagram of the pipe distribution network in Hanoi (the directions on the pipes correspond to the situation marked as * in table 2)

Table 1. Usable standard diameters and corresponding unit costs

\begin{tabular}{|l|l|l|l|l|l|l|l|l|}
\hline Number & 1 & 2 & 3 & 4 & 5 & 6 & 7 & 8 \\
\hline$d_{s}(\mathrm{~mm})$ & 254 & 304.8 & 406.4 & 508 & 609.6 & 762 & 1016 & 1270 \\
\hline$c_{s}(\$ / \mathrm{m})$ & 33.39 & 45.726 & 70.4 & 98.378 & 129.333 & 180.748 & 278.78 & 450.9 \\
\hline
\end{tabular}

Table 2. Pipe lengths and allotted standard diameters [3]

\begin{tabular}{|c|c|c|c|c|c|c|c|c|c|c|c|}
\hline Pipe & $\begin{array}{c}\text { Length } \\
(m)\end{array}$ & \multicolumn{3}{|c|}{$d_{s}$ numbers in the list } & Pipe & \multicolumn{2}{c|}{$\begin{array}{c}\text { Length } \\
(m)\end{array}$} & \multicolumn{3}{|c|}{$d_{s}$ numbers in the list } \\
\hline 1 & 100 & 5 & 6 & $(7)$ & $8^{*}$ & 18 & 800 & 2 & 3 & 4 & $(5)$ \\
\hline 7 & 850 & 5 & $(6)^{*}$ & 7 & 8 & 24 & 1230 & 3 & 4 & 5 & $(6)$ \\
\hline 8 & 850 & 5 & $(6)^{*}$ & 7 & 8 & 25 & 1300 & 4 & $5^{*}$ & $(6)$ & 7 \\
\hline 9 & 800 & 5 & $(6)^{*}$ & 7 & 8 & 26 & 850 & 2 & $3^{*}$ & $(4)$ & 5 \\
\hline 10 & 950 & $5^{*}$ & 6 & $(7)$ & 8 & 27 & 300 & $(1)^{*}$ & 2 & 3 & 4 \\
\hline 11 & 1200 & 3 & 4 & $(5)^{*}$ & 6 & 28 & 750 & $(1)^{*}$ & 2 & 3 & 4 \\
\hline 12 & 3500 & 3 & $(4)^{*}$ & 5 & 6 & 29 & 1500 & 1 & 3 & $(3)^{*}$ & 4 \\
\hline 13 & 800 & $3 *$ & 4 & $(5)$ & 6 & 30 & 2000 & $(1)$ & $2^{*}$ & 3 & 4 \\
\hline 14 & 500 & 1 & $(2)$ & $3^{*}$ & 4 & 31 & 1600 & $(1)$ & $2^{*}$ & 3 & 4 \\
\hline 15 & 550 & $(1)$ & 2 & 3 & $4^{*}$ & 32 & 150 & 1 & $2^{*}$ & $(3)$ & 4 \\
\hline 16 & 2730 & $1 *$ & $(2)$ & 3 & 4 & 33 & 860 & 1 & $2^{*}$ & $(3)$ & 4 \\
\hline 17 & 2750 & 1 & 2 & $(3)^{*}$ & 4 & 34 & 950 & 3 & $(4)$ & $5^{*}$ & 6 \\
\hline
\end{tabular}



AMF

* - values selected in a suboptimal solution with

() - values selected in a suboptimal solution with AG (Popa and Tudor, 2000) [3]

Table 3. Design flows in junctions [3]

\begin{tabular}{|c|c|c|c|c|c|c|c|}
\hline $\begin{array}{c}\text { Nod } \\
\mathrm{e}\end{array}$ & $\left(\mathrm{m}^{3} / \mathrm{h}\right)$ & $\begin{array}{c}\text { Nod } \\
\mathrm{e}\end{array}$ & $\left(\mathrm{m}^{3}\right)$ & $\begin{array}{c}\text { Nod } \\
\mathrm{e}\end{array}$ & $\left(\mathrm{m}^{3} / \mathrm{h}\right)$ & $\begin{array}{c}\text { Nod } \\
\mathrm{e}\end{array}$ & $\left(\mathrm{m}^{3} / \mathrm{h}\right)$ \\
\hline 1 & 805 & 9 & 525 & 17 & 865 & 25 & 170 \\
\hline 2 & 890 & 10 & 525 & 18 & 1345 & 26 & 900 \\
\hline 3 & 850 & 11 & 500 & 19 & 60 & 27 & 370 \\
\hline 4 & 130 & 12 & 560 & 20 & 1275 & 28 & 290 \\
\hline 5 & 725 & 13 & 940 & 21 & 930 & 29 & 360 \\
\hline 6 & 1005 & 14 & 615 & 22 & 485 & 30 & 360 \\
\hline 7 & 1350 & 15 & 280 & 23 & 1045 & 31 & 105 \\
\hline 8 & 550 & 16 & 310 & 24 & 820 & 32 & $1 \overline{9}$ \\
\hline
\end{tabular}

Moreover, it was allowed a frequency chart of the flows stressed from consumers with $\mathrm{K}=5$ classes and the average values per class, respectively the stress durations throughout the year, as shown in table 4.
Table 4. Average consumer flows and demand times, by frequency classes [3]

\begin{tabular}{|c|c|c|c|c|c|}
\hline Class & 1 & 2 & 3 & 4 & 5 \\
\hline Fraction of q & 0.15 & 0.3 & 0.5 & 0.75 & 0.95 \\
\hline Duration (h) & 1463 & 1095 & 4380 & 1095 & 727 \\
\hline
\end{tabular}

\section{INTERPRETATION OF RESULTS}

The best solution among those found with these input data corresponds to total annual costs amounting to 278547,4 out of which $181439,7 /$ year is the cost of the pipes and 97107,7/year is the cost of water treatment at the tank. With a lifespan of $\mathrm{T}=30$ years, the total cost of the pipelines amounts to 5443191 - so it is lower than the best solution obtained with AG. The pipe diameters corresponding to this solution are marked with $*$ in table 2 and - for the sake of a comparison, the diameters of the optimal solution identified using AG in Popa and Tudor, 2000 are marked with parentheses (). It can be seen that, out of the approximately $39,5 \mathrm{~km}$ of pipeline network, $24.23 \mathrm{~km}$ had identical diameters as both the $\mathrm{AG}$ and the AMF formulations [3].

Table 5 shows the pipe flows, loads and chlorine concentrations in nodes for the solution marked with * in the design flow mode.

Table 5. Pipe flows, load and chlorine concentration in nodes in the design flow mode

\begin{tabular}{|l|l|l|l|l|l|l|l|l|l|}
\hline Pipe & $\begin{array}{l}\text { Debit } \\
(\mathrm{m} 3 / \mathrm{h})\end{array}$ & Pipe & $\begin{array}{l}\text { Debit } \\
(\mathrm{m} 3 / \mathrm{h})\end{array}$ & Node & $\begin{array}{l}\text { Pressure } \\
(\mathrm{m})\end{array}$ & $\begin{array}{l}\text { Conc. } \\
(\mathrm{mg} / \mathrm{l})\end{array}$ & Node & $\begin{array}{l}\text { Pressure } \\
(\mathrm{m})\end{array}$ & $\begin{array}{l}\text { Conc. } \\
(\mathrm{mg} / \mathrm{l})\end{array}$ \\
\hline 1 & 19940.2 & 18 & 2453.4 & 1 & 38.24 & 0.271 & 17 & 52.98 & 0.290 \\
\hline 2 & 19050.2 & 19 & 2513.4 & 2 & 99.00 & 0.303 & 18 & 73.54 & 0.296 \\
\hline 3 & 8000.3 & 20 & 7686.4 & 3 & 86.63 & 0.300 & 19 & 79.09 & 0.299 \\
\hline 4 & 7870.3 & 21 & 1415.0 & 4 & 81.99 & 0.297 & 20 & 76.16 & 0.293 \\
\hline 5 & 7145.3 & 22 & 485.0 & 5 & 76.25 & 0.294 & 21 & 47.44 & 0.289 \\
\hline 6 & 6140.3 & 23 & 4996.4 & 6 & 70.29 & 0.289 & 22 & 34.30 & 0.288 \\
\hline 7 & 4790.3 & 24 & 3245.4 & 7 & 64.18 & 0.288 & 23 & 53.71 & 0.286 \\
\hline 8 & 4240.3 & 25 & 2425.4 & 8 & 57.15 & 0.286 & 24 & 48.81 & 0.281 \\
\hline 9 & 3715.3 & 26 & 1041.3 & 9 & 51.65 & 0.283 & 25 & 40.00 & 0.277 \\
\hline 10 & 2000.0 & 27 & 141.3 & 10 & 47.67 & 0.280 & 26 & 31.19 & 0.274 \\
\hline 11 & 1500.0 & 28 & 228.7 & 11 & 43.29 & 0.276 & 27 & 30.52 & 0.267 \\
\hline 12 & 940.0 & 29 & 706.1 & 12 & 40.18 & 0.270 & 28 & 46.55 & 0.278 \\
\hline 13 & 1190.3 & 30 & 416.1 & 13 & 30.96 & 0.250 & 29 & 31.65 & 0.269 \\
\hline 14 & 575.3 & 31 & 56.1 & 14 & 36.83 & 0.278 & 30 & 31.43 & 0.258 \\
\hline 15 & 295.3 & 32 & 303.9 & 15 & 35.07 & 0.275 & 31 & 32.05 & 0.266 \\
\hline 16 & 243.4 & 33 & 408.9 & 16 & 34.90 & 0.269 & 32 & 100.00 & 0.303 \\
\hline 17 & 1108.4 & 34 & 1214.1 & - & - & - & - & - & - \\
\hline
\end{tabular}

It may be seen that the minimum value imposed for the residual chlorine in the network, $C_{\lim }=0,25 \mathrm{mg} / \mathrm{l}$, is reached in node 13 , while the minimum pressure load value of $30,52 \mathrm{~m}$ from node 27 is higher than the limit imposed by $\mathrm{H}_{\min }=30 \mathrm{~m}$. The chlorine concentration necessary when leaving the tank, in the design flow mode, is $C_{R}=0,303 \mathrm{mg} / 1$.
When the network is running, with the average consumption flows by classes as shown in table 4 , it turned out that the critical node in terms of water quality remains node 13 , and in order to ensure in this node 
$\mathrm{C}_{\lim }=0,25 \mathrm{mg} / \mathrm{l}$, the chlorine concentrations

necessary when leaving the tank are shown in table 6 .

Table 6. Chlorine concentration when leaving the tank, by flow classes

\begin{tabular}{|c|c|c|c|c|c|}
\hline Class & 1 & 2 & 3 & 4 & 5 \\
\hline $\begin{array}{c}\text { Average flow in the } \\
\text { network (m3/h }\end{array}$ & 2991 & 5982 & 9970 & 14955 & 18943 \\
\hline $\begin{array}{c}\text { Chlorine } \\
\text { concentration in } \\
\text { tank (mg / })\end{array}$ & 0.863 & 0.470 & 0.367 & 0.323 & 0.306 \\
\hline
\end{tabular}

It can be seen that, for the considered network and input data, only at the lowest consumption flows (15\% of the calculation values) the chlorine concentration necessary when leaving the tank exceeds the upper limit of $0,5 \mathrm{mg} / \mathrm{l}$ stated by law no. 458/2002 [5]. For this specific situation it would be useful to address the issue of finding the optimum positions and chlorine concentrations introduced in the network by additional booster stations, so as it does not exceed the maximum accepted value when leaving the tank and so as it does not fall below the minimum accepted value when it is already in the network [3].

\section{CONCLUSION}

Approaches based on Artificial Intelligence are a strong and very effective tool when modelling water distribution projects. When used, the model can successfully simulate tank emptying, transit flows, node pressures and future conditions. However, this work method based on models identifies the various stresses in order to analyze them and, ultimately, to find the best solutions. This example shown by the two authors can be adapted and calibrated to particular situations, which substantiates decision making, but can also be a reference for addressing the problem using other artificial intelligence techniques.

\section{REFERENCES}

[1] Ianculescu O., Ionescu G., Alimentari cu apa, Editura Matrix Rom, Bucharest, 2002, 127 - 142

[2] M van Dijk, SJ van Vuuren, JE van Zyl, Optimising water distribution systems using a weighted penalty in a genetic algorithm, Water SA Vol. 34 No. 5 October 2008, 537 - 548

[3] Vuţă L. I., Popa R., Model de dimensionare optimă a reţelelor hidraulice, ţinând seama de calitatea apei, IWM Conference (2008), 135 - 145

[4]

https://ro.qaz.wiki/wiki/Ant_colony_optimization_algori thms accessed at 25.02.2021

[5] LEGE nr. 458 din 8 iulie 2002 (**republicată**) 


\title{
THE APPLICATIONS OF GIS FOR DEVELOPMENT SOME CHOOSING AREAS
}

\author{
Baylon M Angelica ${ }^{1}$, Panaitescu M \& Panaitescu F V ${ }^{2}$ \\ ${ }^{1}$ Maritime Academy of Asia and the Pacific, Mariveles Bataan, Philippines, e-mail address:ambaylon@ maap.edu.ph \\ ${ }^{2}$ Constanta Maritime University, Constanta, Romania, e-mail address:mariana.panaitescu@cmu-edu.eu, \\ viorel.panaitescu@cmu-edu.eu
}

\begin{abstract}
This paper introduces Geographic Information System and its applications using datasets and innovative for research, extension services and development. Case study with GIS datasets is available for the Catanduanes Island. This application is good and efficient and will be recommended for actions in building GIS capability of human resources and for an environmentally sustainable and socio-economically Catanduanes support. GIS technology serves for the holistic development of different areas, in our case, for Catanduanes Island.
\end{abstract}

Key words : GIS, catalyst, research, extension, holistic development, island.

\section{INTRODUCTION}

If one is not sure where Catanduanes is, its relative position in Asia may be verified in Google Earth. Its geographic coordinate is at approximately $13^{0}-47^{\prime}$ North Latitude and at $124^{\circ}-26^{\prime}$ East Longitude (Figure 1). The island is about $40 \mathrm{~km}$ wide and $60 \mathrm{~km}$ long, situated in the eastern seaboard of the Bicol Region in Central Philippines [1].

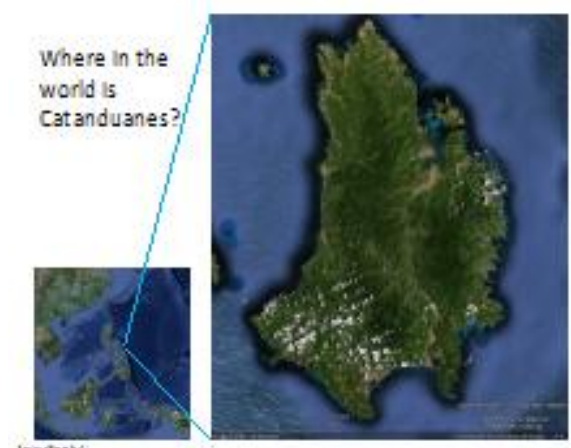

Figure 1 Catanduanes location [1]

The mission of the Province of Catanduanes is holistic. It is similar to that of many other provinces. Pursuing the mission equally requires a holistic approach, looking from the macro level down to micro level of doing things (Figure 2). A versatile technique that can help fulfill this mission with greater success is through the use of GIS.

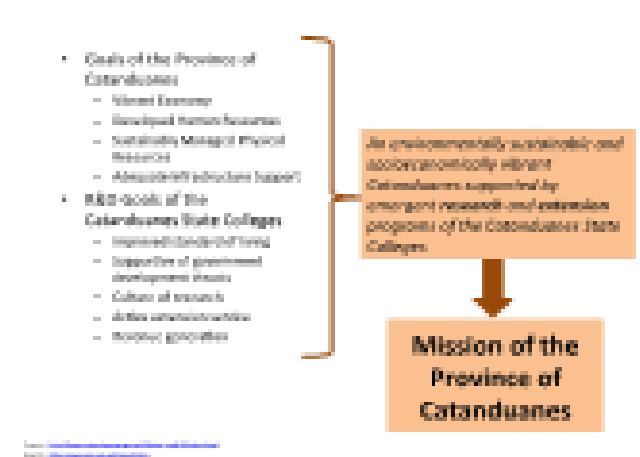

Figure 2. The mission of the province of Catanduanes [2]

The Province of Catanduanes aims for a vibrant economy, developed human resources, sustainably managed physical resources and an adequate Infrastructure Support [2]. On the other hand, the R\&D Goals of the Catanduanes State Colleges are for improved standard of living, supportive of government development thrusts, culture of research, active extension service, and revenue generation [3].

Now where GIS does comes in? How can GIS help in fulfilling the MISSION of Catanduanes? What is GIS? How can Catanduanes benefit from it? This paper answers these questions. Based on degree of applicability, this is a basic (fundamental or pure) type of research which is driven by the researcher's curiosity or interest in scientific questions. The main motivation is to 
expand man's knowledge, not to create or invent something. Based on approach or strategy, this is a grounded theory qualitative research that investigates the quality of relationships, activities, situations or materials wherein the researcher attempts to generate a theory that is grounded in data systematically gathered and analyzed. Hence this study utilized a basic grounded theory qualitative research using the following methods of data collection: internet search, literature search, documentary and content analysis with almost one year of intellectual email query (weekends or evenings) to a GIS specialist (Dr Alejandro Tongco from Oklahoma State University in USA) and marine biodiversity expert (Dr Jimmy Magasca from Catanduanes State Colleges in Virac Catanduanes Philippines). This is also based on the understanding of the researcher (GIS aspirant) having trained on GIS by the GIS expert Dr Alejandro Tongco as mentor during his visit in the Philippines as an invited trainer by MAAP. As part of MAAP and the PAEPI extension services program, GIS with software were shared and conducted to different interested groups as co-sponsors namely : Philippine Navy (former N8 Head Capt Sean Anthony Villa), the Province of Bataan at the Bataan Capitol (former Governor and Mayor Enrique Garcia), the Bataan Peninsula State University ( former President Dr Delfin Magpantay) and the Maritime Academy of Asia and the Pacific ( President VAdm Eduardo Ma R Santos, AFP ( Ret)). The researcher also facilitated the GIS training of MAAP interested students (MRESC and PAEPI members) on three Saturdays at the MAAP campus .

The National Conference on Water and Biodiversity and $3^{\text {rd }}$ Biodiversity Meeting (BIOME3) with theme "Water + diversity $=$ food + life ....." is a challenge to all .

Thinking beyond the above mathematical Biome3 equation, the theme reminds everyone of responsibilities on this world so that future generations can still enjoy this planet earth that will be left behind.

Mathematically, the BIOME3 equation is incorrect. Transposing one variable from the right term to the left term doesn't make any sense. Certainly, the validity of the mathematical BIOME3 equation is not what Dr Aurora Araojo, the BIOME3 Organizing Chair and her BIOME3 Scientific Committee Team (Dr Jimmy Masagca, Prof Estrella Tribiana and Dr Teresita Avila) and colleagues form Catanduanes State University are trying to convey, rather, there are some metaphorical meanings to this BIOME3 equation. One of its definition is provided by this paper. It is believed that in order for "water + biodiversity" to result to "food + life", the BIOME3 equation needs a CATALYST. Hence, a new Biome 3 equation is proposed.

Water + diversity + GIS (Catalyst) $=$ food + life....
GIS can be that CATALYST and can be shown in several ways through the various applications of GIS for Catanduanes, which shall be analyzed and interpreted in this paper on various illustrations.

\section{DISSCUTIONS AND RESULTS}

\subsection{GIS definition:}

Geographic Information Systems (GIS) (4) is a computerized analytical tool that processes geographic data and produces information that helps in making intelligent decisions. These are valuable to planners, administrators, educators, researchers, environmentalists, social scientists, institutional researchers, students, analysts, strategists, and policy makers. It is still evolving since its inception about 40 years ago.

\subsection{GIS , a Convergence of Several Disciplines:}

GIS is a new paradigm, a new way of thinking (Figure 3).

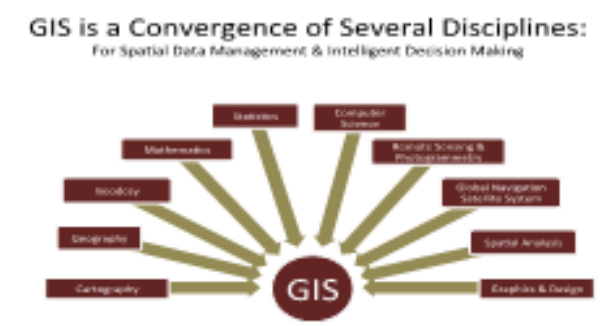

Figure 3. GIS , a Convergence of Several Discipline [4]

GIS is combination of several disciplines including, but not limited to, the following: Geography, Cartography, Geodesy, Mathematics, Statistics, Computer Science, Remote Sensing, Global Navigation Satellite System, Spatial Analysis, and Graphics \& Design, that are used in varying degrees to solve specific problems. GIS analysts must be generalists and must have high level of familiarity of several of these disciplines including his/her own professional background.

\subsection{GIS Applications by Discipline:}

Whatever field of specialization or disciplines, GIS can be applied to it. Some of the headings and subheadings below (particularly those underlined) in relation to the BIOME3 conference may be applicable to Catanduanes Province. 
Agriculture (Agricultural Economics , Agricultural Engineering Agronomy, Farm and Ranch Management, Pest Management and Veterinary Science); Architecture (City, Community, and Regional Planning, Landscape Architecture, Urban and Environmental Design); Business ( Banking and Financial Service Business Administration, Decision Support Systems, Economic and Management Research , Asset and Facilities Management, Marketing ,Media and Press, Operations Research, Real Estate Management and Retail); Defense, Security, and Intelligence ( Defense and Force Health Protection, Enterprise GIS , Geospatial Intelligence (GEOINT), Installations and Environment, Military Operations); Education (Campus / Multi-campus Management, Continuing and Distance Education, Educational Management and Administration, Elementary and High School, Institutional Research, Extension Planning, Vocational and Technical Education, Alumni Management, GIS Science \& Technology Curriculum Development, Research, Theses, \& Dissertations); Engineering (Aerospace Engineering, Chemical Engineering, Civil and Sanitary Engineering, Computer Engineering, Electrical Engineering ,Environmental Engineering, Geomantic / Geodetic Engineering / Surveying, Industrial Engineering, Mechanical Engineering , Mining Engineering); Government (National, Regional, Provincial, Local, Economic Development, Elections, Land Administration, Public Works, Urban and Regional Planning ); Law ( Real Estate , Jurisdictional Law); Libraries \& Museums (Government Documents, Map and Imagery Collections); Mapping and Charting (Aeronautical , Cartographic Publishing , Nautical , Spatial Data Infrastructure);Natural Resource Management (Conservation. Environmental Management, Fisheries, Forestry, Parks and $\underline{\text { Recreation, Petroleum, Range Management. Wildlife }}$ Management and Water Resources Management); Natural Sciences (Biostatistics, Botany, Conservation Biology, Entomology, Marine Biology, Zoology, Ecology, Environmental Science, Oceanography and Coastal Studies and Soil Science); Physical Sciences (Applied Physics, Climate Change, Computer Science, Geology, Geosciences, Earth Science, Geographic Information Sciences, Geochemistry , Hydrology, Paleontology , Quaternary Research , Seismology Research , Meteorology and Climatology); Public Health and Medicine (Environmental Health, Epidemiology, Hospitals and Health Systems, Managed Care, Public Health); Public Safety (Computer-Aided Dispatch, Criminal Justice Criminology, Emergency/Disaster Management , Homeland Security, Law Enforcement, Fire and Rescue, Emergency Medical Services); Social Sciences (Area and Ethnic Studies, Anthropology and
Archaeology, Communications and Journalism, Economics, Geography, Historic Preservation International Studies, Political Science, Public Administration, Psychology, Sociology, Demography ; Travel and Tourism); Transportation (Aviation, Highways, Logistics, Railways, Ports and Maritime and Public Transit); Utilities and Communications (Electric, Gas, Location-Based Services, Pipeline .Telecommunications, Water/Wastewater)(5)

These are only partial list. Almost all disciplines can benefit from GIS. GIS has almost unlimited applications in many disciplines. As Roger Dangermond (founder of the ESRI, the maker of ArcGIS) has said, "The applicability of GIS is limited only by the imagination of those who use it."

As GIS is applicable to any disciplines, hence, GIS maybe used for Government, business, and research on environmental resource analysis, land use planning, locational analysis, Tax appraisal, utility and infrastructure planning, Real estate analysis, marketing and demographic analysis, habitat studies, and archaeological analysis; for Natural resources management like wildlife habitat, wild and scenic rivers, recreation resources, floodplains, wetlands; agricultural lands, aquifers, forests.; for Facilities management like locating underground pipes and cables; Balancing loads in electrical networks; planning facility maintenance, tracking energy use; for Land management like zoning and subdivision planning, land acquisition; Environmental impact policy, water quality management and Maintenance of ownership.; for Street-networks like location analysis, site selection; and development of evacuation plans.

\section{$2.4 \quad$ The GIS process}

The GIS process is made up of several components called inputs (spatial and non-spatial data, GIS software, Computer hardware, manpower and methods and processes). These data are processed (collected, recorded and manipulated/, stored, managed and retrieved, analyzed and modeled, displayed). The System produces useful information (which may be recycled) to produce specifically desired information (Figure 40 [5]. 
GIS datasets to be chosen are dependent on the

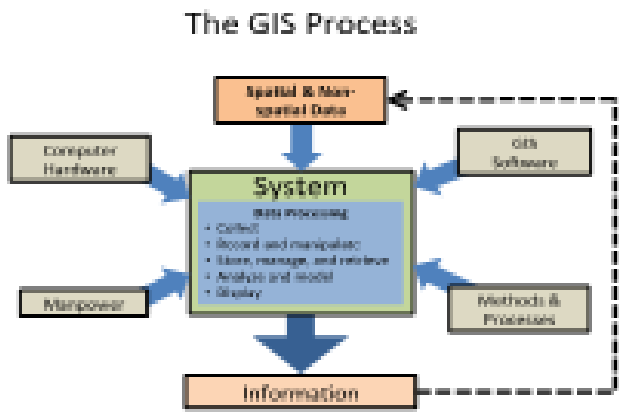

Figure 4. The components of GIS process [5]

organization, then its name can be written only once.

The font for the name of the organization is Times New Roman 10.

\subsection{Data input to GIS}

The GIS system needs data to be processed into useful information. Examples of input data are the following: digital maps, GPS readings, remotely-sensed imagery, tabular data, field survey data, digital products, texts etc (Figure 5)[6].

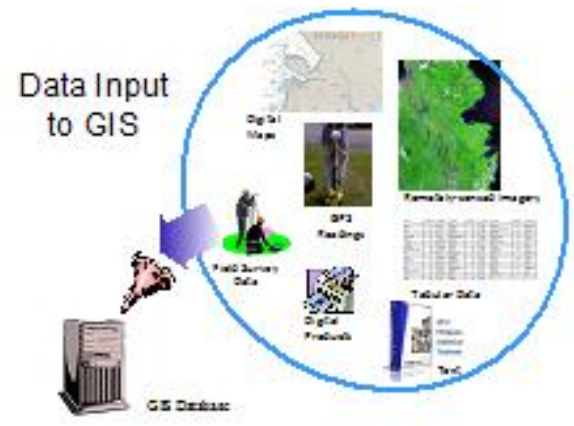

Figure 5. Data input to GIS [6]

The titles of sections and subsections will be aligned left and numbered consequently.

Terms that describe GIS have been repeatedly cited for emphasis and not for redundancy. This is done purposely to embed the concept of GIS in people's consciousness. GIS is a new paradigm - a new way of thinking. To some, if not to many of view, GIS may have a steep learning curve as illustrated by various data layers (land cover, structure, boundaries, hydrography, geographic names, transportation, elevation and orthoimagery.

The GIS project objective dictates the combination of datasets to collect.

\section{$2.5 \quad$ GIS datasets}

You can add or remove, zoom in or out, at will using GIS software. Each dataset contains an attribute table. 
Raster datasets may not readily show an attribute table, but they can be processed to produce attribute information.

Location is the key word in GIS. Without "Location" component, GIS is of no use.

Having common characteristics, almost all problems have a locational component. Problems involve people, location of people, things in those locations, events and phenomena in that location, and relationships of these components. These components and their relationships lend well to scientific GIS analysis and thus intelligent decisions. Hence, a challenge for Catanduanes

\subsection{Benefits for Catanduanes in using GIS}

Certainly, the use of GIS in the Province of Catanduanes would result to: Better Decision Making (Better decisions about location whether in research or development work and common examples include evacuation planning, conservation, natural resource extraction, school facilities management, etc. ) Making the right decisions about a location is critical to the success of an organization or project, e.g. in local governance, management of academic institutions, etc; Improved Communication (GIS-based maps and visualizations greatly assist in understanding situations and in chronicling. ) GIS results are a type of medium that improve communication between different project teams, departments, offices, professional fields, and the public.; Better Record Management (Maintains authoritative records about the status and change of geographic events and also Easy query and retrieval of spatial data that are centrally managed) Geographic records gives comprehensive transaction support and reporting.They are a valuable aid for fulfilling government and accreditation requirements.;Managing Geographically (Essential to understanding: what is happening - and what will happen - in a particular geographic location). Problem in understanding is necessary to make the correct prescription for action. Managing geographically is a new approach to management - taking into account the inherent geographic or spatial nature of things. The Overall Result: is increased efficiency and cost savings. [6]

\section{METHOD AND RESEARCH}

\subsection{Available Base GIS Data for Catanduanes}

To download free data, visit the website www.philgis.org [7] which is made available especially to GIS users of the Philippines. The following data maybe use for GIS-based research and development activities:
Province, Towns, and Barangays for use in population and demographic studies, student distribution studies, etc;

- Elevation data: DEM (digital elevation model, 30-m resolution) for use in delineation of protected areas, road construction planning, watershed delineation, river volume forecasting, etc ;

- Landsat ETM+ with 3 multi-spectral bands (30-m resolution) and panchromatic band (1-m resolution) for use in land-use and landcover studies, calculation of vegetative coverage, coastal resource management, etc.

- Bathymetry: 1-km resolution for use in marine studies, e.g. delineation of marine sanctuaries and protected areas and

- Rainfall for use in studies on climate change, erosion,flood disaster mitigation, etc.

\subsection{Selected GIS Applications/Uses for CatanduanesTables}

Form the BIOEME3 theme; some applications of GIS that are applicable to Catanduanes have been identified. Basic function of GIS is for mapping [8]. GIS may be applied for the social sciences; for campus facilities management; for assessing land cover and coastal resource; for protected area development; for disaster preparedness; for flooding simulation; for bird conservation ; and for navigation and marine resource management .

There are countless other GIS applications. But these cited examples are in relation with the conference BIOME3 theme and is believed, are most needed for the province and its higher educational institutions.

\subsection{Use of GIS for mapping}

Mapping is just one many basic uses of a GIS.

The first (1st) map at the top-left is the boundary map of Catanduanes Island (Figure 8-1). The second (2nd) map is that of Catanduanes and its municipalities (Figure 82 ). The third (3rd) map displays the municipalities applied with coloring scheme and labels (Figure 8-3). The fourth (4th ) map displays the municipalities overlayed with barangay boundaries with labeling (Figura 8-4). These maps or part thereof are valuable in many phases of the GIS project: from planning to implementation to analysis to reporting to evaluation (Google Earth view of the Catanduanes State Colleges campus, Virac Campus, Taken 2010). 


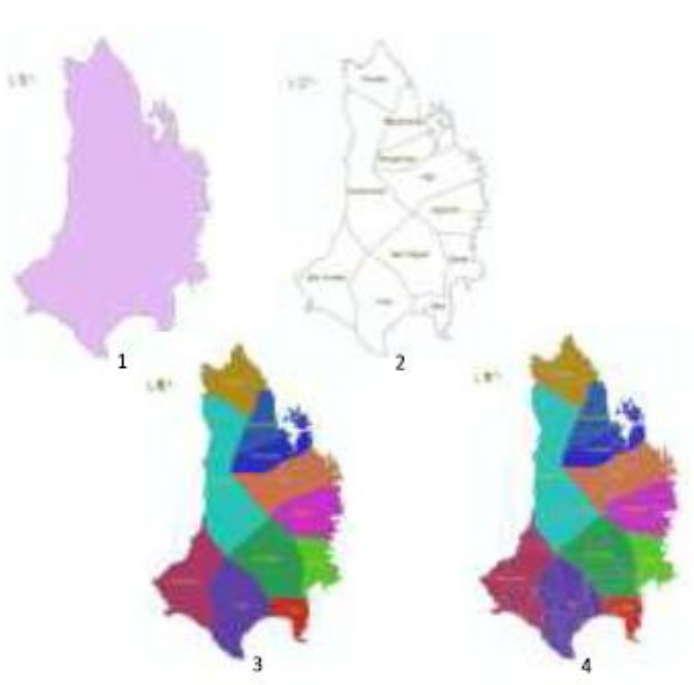

Figure 8 GIS for mapping

\subsection{Use of GIS for social sciences}

Focusing on one municipality in Catanduanes - the Municipality of Bato (Figure 9). Initially, color and labeling may be applied for its barangays.

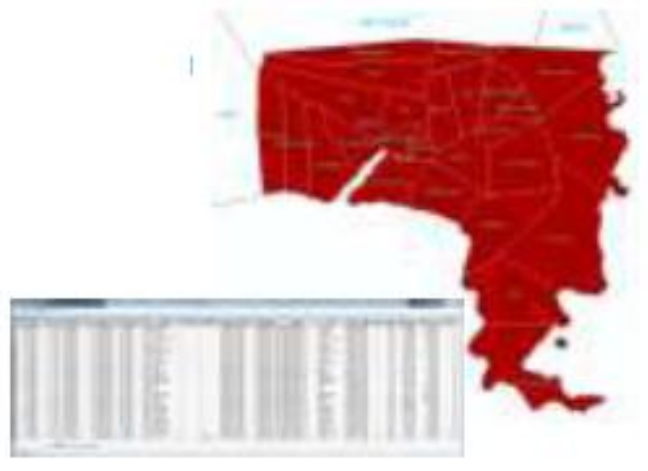

Figure 9 GIS for social sciences

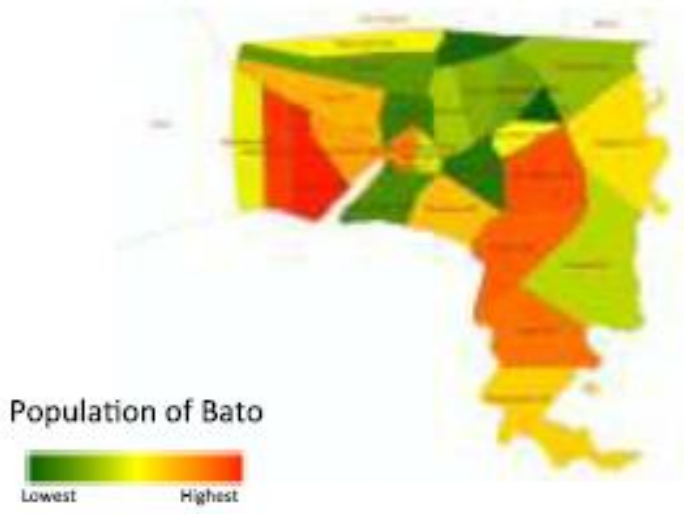

Figure 10 The Bato dataset

The Bato dataset contains a population field for the different barangays (Figure 10). The 2007 population of
Bato is 18,738 distributed over 4,582 hectares. Basic statistics such as sum, mean, and median can be calculated instantly using GIS software. The illustration above also labeled the population value per barangay. In this case, the highest is Bgy Cabugao with 2888 inhabitants and the lowest is Bgy Santa Isabel with 123 people. The picture obviously can give clues to politicians which barangays to prioritize their campaigns. (If the number of voters are included in the table, it could also be mapped and thus show a much better support for political planners.) DOH and DSWD personnel, as well as CSC researchers, can also be guided which barangays to focus their outreach programs.

A better measure of population is population density (i.e. number of people per unit area) this illustration shows the density distribution of barangays in Bato, in

number of people per hectare (Figure 11). Red area (Bgy Ilawod) is the densest; green area (i.e. Bgys Buenavista, Cagraray, and Carorian) is the least dense. We can assume that Bgy Ilawod is the heart of Bato municipality. Again, the illustration can guide govt agencies, as well as HEIs, in planning for social and economic programs in the barangays. GIS analysts and planners may want to know why the eastern barangays are least dense. One might ask: What are the reasons behind it? Is it because of climatic conditions, agriculture, or other reasons? People may quickly guess for the reasons.

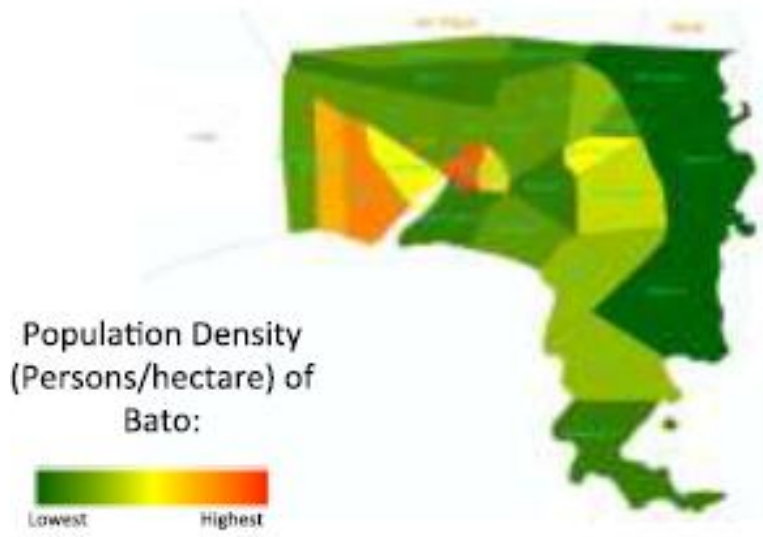

Figure 11 Population density of Bato

However, answers without supporting data or information are almost hearsay. The GIS analyst can help you find concrete evidence by overlaying several datasets, e.g. climate or rainfall, land cover, soils, 


\section{Journal of Marine technology and Environment Year 2021, Vol.I}

diseases, and other datasets in order to come up with objective analysis to justify the implementation of development programs.

\subsection{Use of GIS for campus facilities management}

This a close-up view of the campus of the Catanduanes State Colleges displayed by Google Earth (Figure 12) [9].

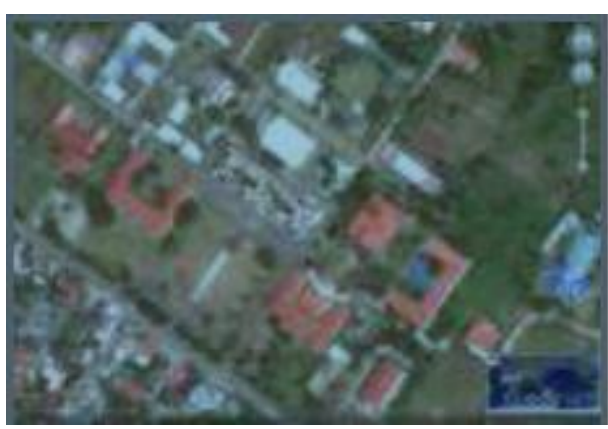

Figure 12 the campus of the Catanduanes State Colleges by Google Earth

One can see the building roofs and thus the footprints. Fortunately for GIS people, GIS datasets can be derived by digitizing (i.e., tracing a point, line, or polygon) the building footprints. To do this, we can convert the Google Earth KML file into a GIS shapefile, which can be done by most GIS software. Building footprints can also be scanned or digitized from building plans and then georeferenced (i.e. tagged to real-world coordinates). Most GIS software can do this conversion and georeferencing. Furthermore, room division lines may be traced for each building footprint. The resulting attribute table - which initially is empty - can then be populated with data such as number and specs of computers and other equipment, office furniture and fixtures, number of personnel, etc. Once the data are stored in a central database, the CSC President Dr Minerva I. Morales or a researcher can quickly query, retrieve,process, or print information about a certain room or all rooms in the campus and their assets. The ease in retrieving this data is valuable also to government such as CHED and TESDA, and accreditation groups, which are interested about such information. Similar process can be done to collect and manage data about the natural resources and other physical facilities of the campus such as water, sewer, telephone, and electrical lines; monuments; benches; trees; and others.

\subsection{Close-up of Virac area: Overlay of Landsat} imagery (CA year 2000), roads, and municipal boundaries

Roads and streets (yellow line), town boundary (white line) overlayed on Landsat imagery (Figure 13).

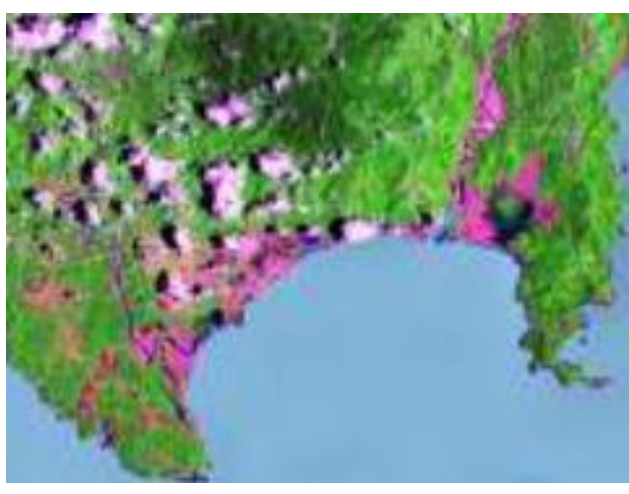

Figure 13 The Overlay of Landsat Virac imagery

Note again the cotton-like features of clouds. The dark areas left of the clouds are the clouds' shadows. The pink areas are urban or developed areas. The dark blue areas are water bodies such as rivers, fishponds, or cleared mangrove areas.

These have to be "ground-truth"to validate their presence.

\subsection{Use of GIS For Assessing Land Cover and Coastal Resource}

Here's a colored rendition of the Catanduanes terrain near Virac (Figure 14). Maps may present a good visual view of the landscape.

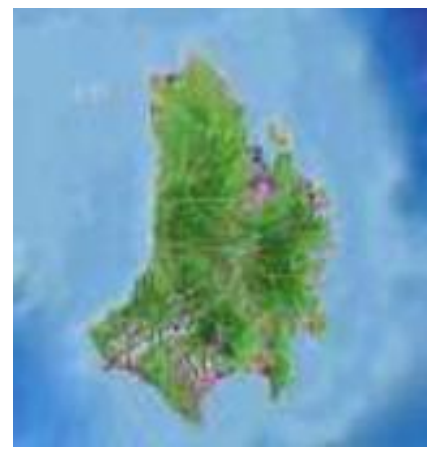

Figure 14 The Catanduanes terrain near Virac

But how useful is this to your GIS study? GIS is much more than just creating beautiful maps. Using the elevation map, you can delineate the certain elevations at certain location to be designated as protected areas.

Overlaying this with the Landsat image and barangay boundaries can give better information about land cover viz-a-viz selection of protected area and elevation, which is valuable for environmentalists and biodiversity specialists. Also, you can delineate the watershed(s) of the rivers that feed into Virac poblacion. 
This task is useful if you want to project the flooding that might occur in the city, the number of people affected, houses and building most probably flooded or destroyed, etc. Note that data layers can be added or removed at will with the click of a mouse. But first you have added this data to the GIS and make the necessary processing steps to display them nicely as you would want it.

\section{$3.8 \quad$ Use of GIS for protected area development}

This is an overlay of several GIS datasets: Administrative boundaries of towns and barangays overlaid on Landsat satellite imagery which in turn is overlaid on bathymetry (Figure 15). Land use / land cover is depicted by the Landsat image in various colors, e.g. dark green stands for forest cover, light green means thin forest cover or farm lands, red color is urban populated areas or absence of vegetation. The white cotton-like features are clouds. The dark areas beside the clouds are cloud shadows. From this map, planners can assess the extent of a particular land cover or land use, which barangays still have forests, which barangays have manmade structures such as fishponds, etc. GIS is about describing and analyzing the spatial relationships of the various data layers present in the map. The map also shows the variation in ocean depth depicted by graduated blue color.

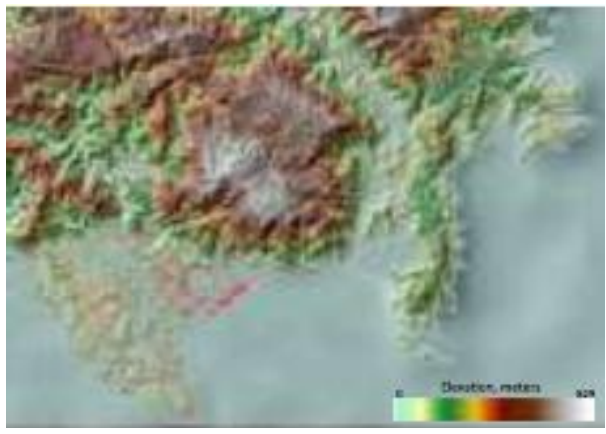

Figure 15 GIS for protected area development

The Landsat and bathymetry data used for this map are of low resolution and thus for approximation only. For critical studies, high-resolution imagery is needed to give detailed output.

\section{$3.9 \quad$ Use of GIS for disaster prepasredness}

GIS mapping and analysis is very important in dealing with disaster preparedness and mitigation. Several data layers are used here: provincial, municipal, and barangay boundaries as well as roads, overlaid on digital elevation model (Figure 16).

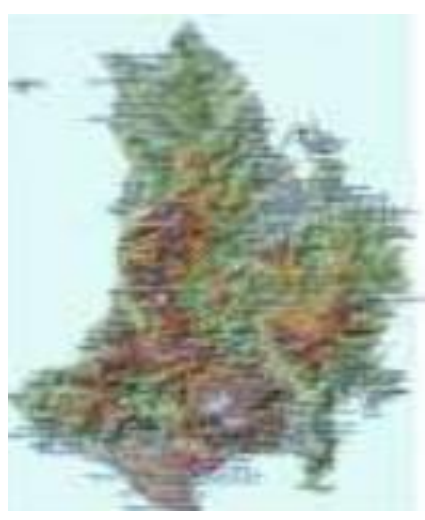

Figure 16 GIS for disaster preparedness

Which coastal barangays do you think would receive the most devastating hit if a tsunami occurs somewhere in the Philippine Trench?

It's Mother Nature's will, that Catanduanes is situated along the typhoon path. (God has positive reasons for making it so, which only few of us may be aware of.) Nothing can be done about it, but we can cushion the destructive effects of natural calamities if we make the necessary mitigating measures. Basically, GIS can help produce the maps that can simulate typhoon path, its strength, and the population that may be affected. People can be warned about areas that may be prone to landslides or flooding.

Let's think of climate change. How many of the coastal communities do you think will be submerged and how much? How will the rise in sea level affect the coastal communities of Catanduanes? Which barangays are most vulnerable? How many people will be affected? These are issues that can be better-addressed using GISengaged research by CSC and other institutions in Catanduanes.

On the questions why floods occur. These are some of the causes: Climate change ; Big waves; Continuous rainfall; Swollen rivers; Tsunamis; High tide; Man-made structures, negligence, indifference and Combination of the above. Climate change (high temperature) can cause water volume expansion, raising the sea level. To look at a simulation of coastal flooding, let's focus at some municipalities in northern Catanduanes.

3.10 View of Panganiban and Viga Municipalities, Northern Catanduanes, derived from Digital Elevation Model Data

This is what it may look like during normal days. Note the elevation legend at the lower right corner, ranging from a light blue ( 0 meter) to dark red to white (829 meters)(Figure 17). 


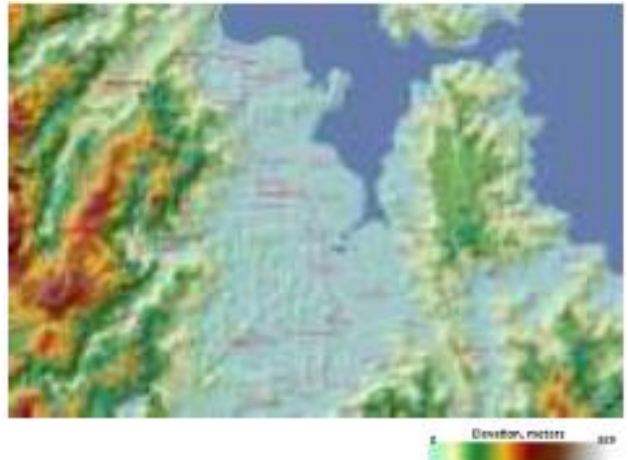

Figure 17 View of Panganiban and Viga Municipalities, Northern Catanduanes

Note the municipal and barangay boundaries, and the relative locations of the hills and mountains within the barangays.

\subsubsection{Flooding Simulation: 5-meter water level rise}

This is a simulation of coastal flooding when sea level rises to 5 meters. Note the flooding extent in the coastal barangays (Figure 18). Note also that pockets of water bodies inland have started to appear. Although flooding may appear small on the map, the actual flooded areas can be larger. The map shows which barangays to warn before a actual floods occur. Based on the simulation the number of people affected can also be estimated. How is the map made?

The map is an overlay of several GIS datasets, namely:

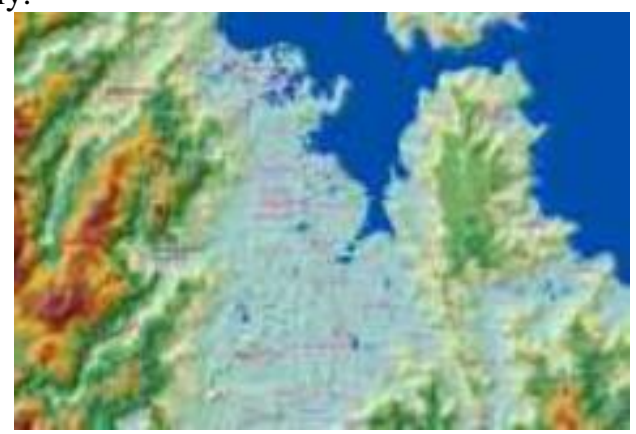

Figure 18 Flooding simulation-5 meter water level rise

DEM (digital elevation model) - elevation values were categorized and represented with graduated colors; Municipal and barangay boundaries (white line) this is to determine the relative location of flooding in the barangays and

Roads (red line) - this is also added to visualize which portions are flooded. This is important to know for emergency evacuation purposes. Other layers may be added such as churches, cemeteries, government buildings, etc.

\subsubsection{Flooding Simulation: 10-meter water level rise}

Now, this is getting scary. You can see which barangays are submerged (Figure 19).

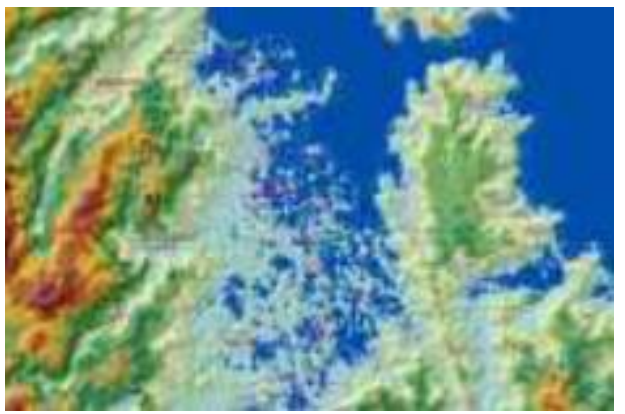

Figure 19 Flooding simulation-10 meter water level rise

Is there someone from the audience coming from any of these barangays or towns? Some of you may think your town or barangay is not affected because it's not shown on this map. Well, you may be wrong. Let's look at a different location of Catanduanes on the next.

\subsubsection{Flooding Simulation: 10-meter water level rise in San Andres and Virac}

Flooding in urban areas certainly exact a higher toll in terms of lives, property, displacement, and other problems (Figure 20).

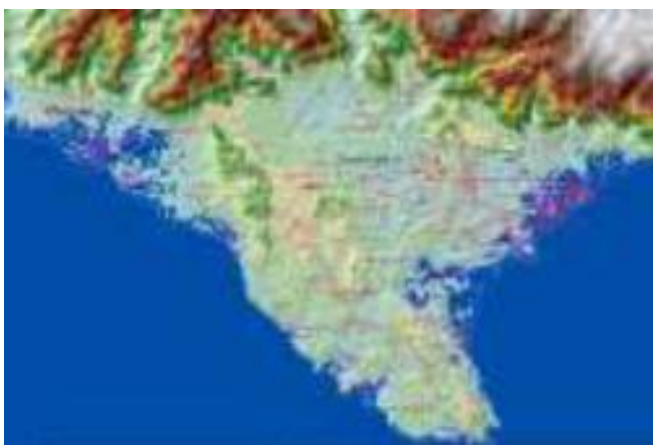

Figure 20 Flooding simulation-10 meter water level rise in San Andreas and Virac

Economic assessment of devastation could run into millions or even billions of pesos. So what are we going to do next? You may think flooding could never occur this high? How sure are you? The 2011 Tohoku earthquake and tsunami in Japan killed 16,000 people and the economic cost of devastation was estimated at US\$235 billion according to the World Bank. The 2004 


\section{Journal of Marine technology and Environment Year 2021, Vol.I}

South Asian earthquake and tsunami killed over 230,000 people from Sri Lanka, Maldives, India, Indonesia, and Thailand. We are lucky (at least for the time being). When are we going to end procrastination? There's no doubt that coastal barangays are most vulnerable to this kind of disaster. GIS certainly can help in planning and implementation of disaster prevention and mitigation programs.

\subsection{Use of GIS for bird conservation}

Let's talk something less scary.The use of GIS for Bird concervation. The illustration shows the point locations of bird sightings in the Canticol and Mt. Hilong-hilong area of Agusan del Norte province (Figure 21).

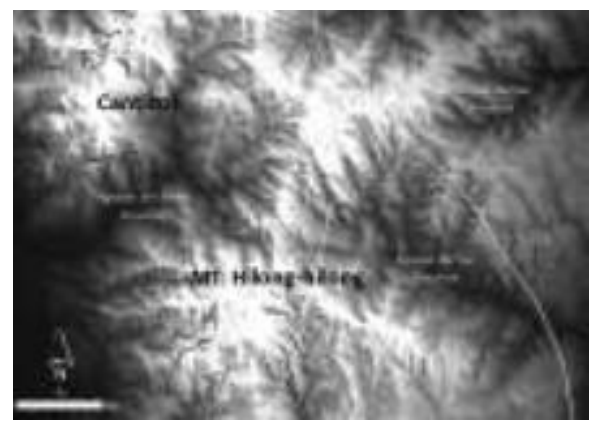

Figure 21 GIS for bird conservation

Although the sightings were of different bird species, the points are all colored black for the black-and-white illustration. In GIS, the points called GPS, which contain latitude-longitude coordinates of birds can be overlaid on a digital elevation map as above. The relative location of the sighting points with each other and with the terrain are shown. Using the scale bar as shown, one can deduce the relative distances of the points and their distance to the provincial boundaries. The information of bird sightings can be used in studies such as bird conservation planning and protected area development.

\subsection{Use of GIS fornavigation and marine resource management}

The map is a hillshade or relief map of Catanduanes overlayed on a bathymetry layer (Figure 22).

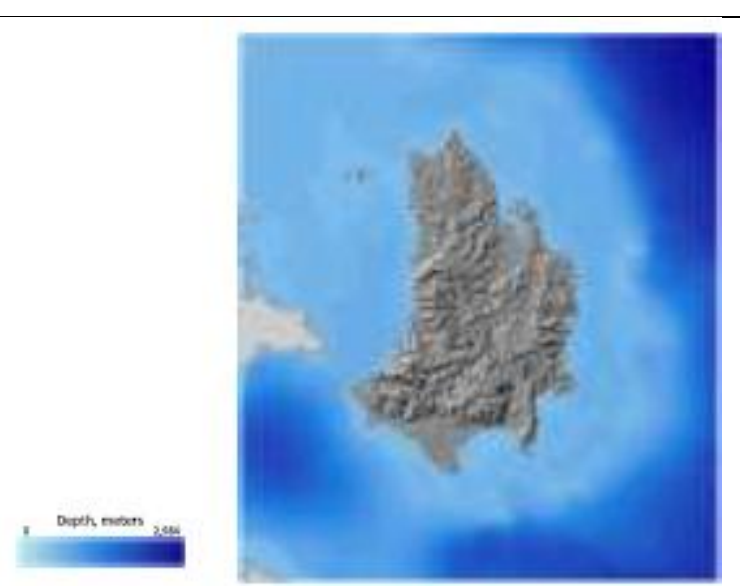

Figure 22 GIS for navigation and resource management

Note the graduated blue color and depth in meters. Catanduanes is closer to the northern vertex of the northsouth elongated Philippine Trench along the eastern coast of the Philippines. The deepest point is $10 \mathrm{~km}$. Research on navigation and coastal resource management entails, among other things, conducting GIS-based bathymetric mapping of the coastal waters of the island. Existing bathymetric data is a rough $1-\mathrm{km}$ resolution and can be downloaded free from the General Bathymetric Chart of the Oceans (GEBCO). The processed result is show in the illustration in front of you. This resolution can do location approximations to do studies, such as marine protected area delineation; spatial analysis of coastal marine life; bathymetry of harbors and approaches; etc. However, higher-resolution bathymetric map is needed for greater detail and clearer representation of the undersea terrain, and thus for better analysis of study results. There are paper nautical charts (old, about 50 years old) of the Philippines produced by the U.S. National Imagery and Mapping Agency (NIMA) [now called National Geospatial-Intelligence Agency (NGA)] containing depth sounding values that could be georeferenced, digitized, converted to point GIS vector files, and mapped as in the illustration above. Please see list of nautical charts at http://www.charts.noaa.gov/NGAViewer/nga9.pdf [5].

Unfortunately, the nautical chart for Catanduanes (NGA Chart 91040) is unavailable for download or viewing from the site. A smaller scale is available for viewing: NGA Chart 91030 at 398,000 scales. Again, these points have to be "oceanfloor-truthed" using recent and detailed bathymetric mapping of the area of interest in order to validate the depth data to be used for research. On the other hand, coastal resource management studies need higher-resolution satellite or other remotely-sensed multispectral imagery to assess the distribution and health of coastal vegetation. Temporal changes of coastal vegetation can be examined from older multispectral imageries and compared with 


\section{Journal of Marine technology and Environment Year 2021, Vol.I}

the more recent ones. These entails image analysis on a GIS software in order to assess other related information such as reduction (or increase) in vegetative (or urban) land cover.

\subsection{Depth Contours and Labels (Depth Values)}

Here's a similar rendition of sea depth. Vector contour lines were created based on GEBCO 1-km resolution bathymetric grid map and overlayed on the raster bathymetry from the same raw data (Figure 23). These are approximate contour lines and depth values that need to be validated by a more accurate bathymetric data collection and mapping method.

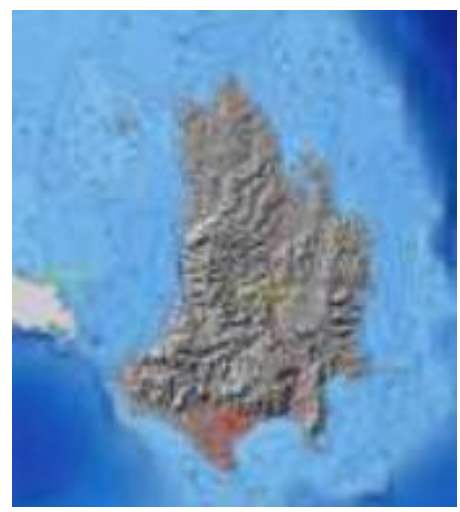

Figure 23 Depth values

Depth labeling of contour lines definitely adds value to the data for viewer. Note that the illustration is an overlay of 5 GIS datasets or layers. Starting from the bottom - Bathymetric Map, Provincial Map of the Philippines (only portion of Camarines Sur is shown; Catanduanes provincial map is also covered by elevation map), Digital Elevation Map of Catanduanes, Contour Lines, and Roads). Any combination of these or other GIS data layers can be used in navigational and coastal environmental research.

With labeling of contours, water depths around the islands are known [11]. Note that the contour lines are drawn at 20-m interval. Widely spaced contour lines suggest gradual depth. Farther from the coasts most notably $15 \mathrm{~km}$ to the northeast and also southwest, depth suddenly

drops like a ravine, as can be seen by the closely spaced contour lines.

\subsection{A view from Google Earth}

What's the difference between Google Earth image and Landsat imagery? Google Earth nowadays presents stunning high resolution true-color pictures of many parts of the country (Figure 24). But the picture is hardly suitable for image analysis.

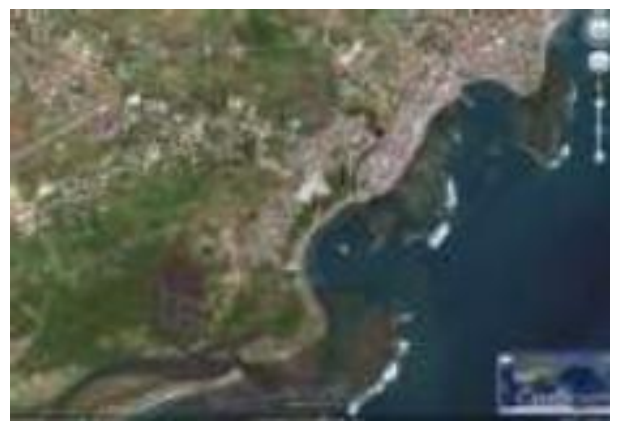

Figure 24 A view from Google Earth

Landsat imagery or multispectral imagery consists of several bands that allow manipulation of the bands by the image analyst to emphasize visual clarity of events such as floods, landslides, forest fires, diseased vegetation, etc. Landsat imagery is available for free at low resolution (30 meters or more). High-resolution imagery (e.g. 10 meters or less) are expensive, about 1 US dollar per sq.km. Points, lines, and polygons can be traced on the imagery, saved as KML file, and converted to GIS files. In contrast, GIS files can be converted to KML files and overlaid in Google Earth. The image itself can be "screenshot", geo-referenced, and used as an overlay on the GIS display.

However, be warned that there may be copyright restrictions regarding this.

\section{CONCLUDING REMARKS}

It can be surmised that GIS is a powerful innovative tool for analyzing the data. It provides answers to the following point of interest like: What's happening inside a certain location? How to prepare for something that will occur within a certain radius or distance What to expect from nearby after finding out what's occurring within a certain radius or distance. Behind the beautiful maps are stories. So what do these maps convey and how do they challenge the analyst, aside from their esthetic value? Using GIS, the beautiful maps produced provides better visualization of project process; easier manipulation of data layers, greater insights on the phenomenon involved and resulting scenario; critical analysis of the scenario and enhanced intelligent decision making to challenges related to the socio-economic development and environmental sustainability of Catanduanes. Hence how should GIS comes into the BIONE3 equation

$$
\text { "water+biodiversity=food+life"? }
$$


GIS should therefore come into the equation as an emphatic exclamation point (GIS!), because indeed GIS is a unique positive catalyzer.

\section{"water+biodiversity $=$ food+life"}

Using GIS to confront real-world problems can lead to intelligent and informed decisions concerning management of environment and natural resources resulting in sustenance of the present and future generations of Catanduanes. This belief must be validated by the Province of Catanduanes by applying GIS in its various data sets to be convinced that the benefits of GIS presented on this paper are real [10], [11].

\section{RECOMMENDED ACTIONS}

For the research, extension services and development of the Province of Catanduanes, this would still be dependent on the quality of its human resources thru GIS capability building for the Catanduanes State Colleges and Catanduanes researchers and planners .

Hence the following actions are recommended. Conduct GIS Training and Workshops; (Use free GIS software to promote GIS knowledge dissemination: Several good ones available, e.g. MapWindow GIS, Quantum GIS, SAGA GIS, DIVA GIS, etc; Invite a trainer that understands local needs and challenges, has a multidisciplinary background, and is open to posttraining questions; Use local data samples during the training to make it as an on-job-problem solving exercise; Install Internet connection if available; if none, that's fine; training can be done without Internet; Bring a capable laptop or desktop computer (at least 2 Gig memory and $100 \mathrm{MB}$ hard drive capacity) );

Initiate and participate in GIS conferences, meetings, lectures, etc; (Network and share knowledge with other GIS enthusiasts like you; Conduct GIS conferences, meetings, lectures, etc. Start in-house, then do local, provincial, regional, etc.);

Apply GIS to your workplace decision-making whenever possible (Learning and applying GIS can be daunting at first, but once you learn to do it, the next challenges become far easier ).

Start a simple GIS-based research project; Collaborate and consult with like-minded GIS persons within CSC or Provincial Capitol. "Two heads are better than one." Call or email the Trainer if you need advice!; Include GIS goals in your Strategic Development Plan to institutionalize GIS and to justify budget allocations for future GIS-based projects and activities; Detail specific GIS-based activities, Start a GIS newsletter; Make a webpage for GIS-based project within the institution's website;

Build a central GIS database for the province; (Collaborate in GIS data buildup to avoid data duplication efforts, share costs in data buildup, thus minimize overall cost); and

Seek collaborations!You may insert acknowledgements in the paper if necessary before the references section.

\section{REFERENCES}

[1] Google earth of Catanduanes Province, Taken 2012.

[2] Catanduanes | Explore the Island of Catanduanes retrieved on 10th January 2015 at

http://www.catanduanes.gov.ph/vision andmission.html

[3] Catanduanes State Colleges - About CSC retrieve on 10th January 2015 at

http://www.csc.edu.ph/about.htm

[4] Geographic Information Systems as an Integrating Technology retrieved on $10^{\text {th }}$ January 2015 at http://www.colorado.edu/geography/gcraft/notes/intro/in tro.html.

[5] nga9.pdf (application/pdf. Object) retrieve on 10th January 2015 at

http://www.charts.noaa.gov/NGAViewer/nga9.pdf

[6] Overview | Geographic Information Systems retrieved on $10^{\text {th }}$ January 2015 at

http://www.gis.com/content/top-five-benefits-gis.

[7] PhilGIS (Philippine GIS Data Clearinghouse) retrieve on 17 Feb 2012 at www.philgis.org

[8] Esri Industries | GIS Mapping Solutions for Industry retrieved on $10^{\text {th }}$ January 2015 at

www.esri.com/industries.html

[9] Google Earth view of the Catanduanes State Colleges campus, Virac Campus, Taken 2012

[10] Dr Alejandro Tongco, Dr Jimmy Masagca Exchanged email communications with GIS Specialist (al.tongco@okstate.edu;(pacifictechjtm@yahoo.com )

[11] LEMENKOVA POLINA, R scripting libraries for comparative analysis of the correlation methods to identify factors affecting Mariana trench formation, Jounal of marine Technology and Environment, pp 3544, vol 2, 2018. 


\title{
EVALUATION OF THE QUALITY STANDARDS OF THE WATER DISCHARGED INTO THE SEA AFTER WASHING THE EXHAUST GASES
}

\author{
Cojocaru Remus-Cristinel ${ }^{1}$ \\ ${ }^{1}$ Constanta Maritime University, Doctoral School of Mechanical Engineering and Mechatronic, 104 Mircea cel Batran \\ Street, 900663, Constanta, Romania, email costi.cojo@yahoo.com
}

\begin{abstract}
The main objective of this paper is to propose technical solutions for monitoring and recording data for a gas washing system on board a ship and to maintain in standard parameters the quality of surface water following the discharge of gas washing water from installations from the ship. The specific objectives of achieving the general objective are: the methods for evaluating the laboratory samples from the washing water evacuation, modeling the dynamics of the washing water and modeling the dispersion of the scrubber washing water on board for a case study,evaluation of the quality standards of the water discharged into the sea after washing the gases, the elements of calculation, modeling and graphical representation are present.
\end{abstract}

Key words : discharge, evaluation, exhaust, gas, quality,sea,standard, water.

\section{EVALUATION OF LABORATORY SAMPLES FROM WASHING WATER DISCHARGE}

The continuous monitoring of the required parameters of the exhaust gas cleaning system (EGCS) is used, depending on:

- IMO requirements (MEPC.259 (68) 2015 Guidelines for exhaust gas cleaning systems) EU Sulfur Directive (EU / 2012/33;

- All ships are equipped with continuous monitoring equipment to automatically record the required parameters;

- The IMO encourages regular monitoring to test additional parameters with on-site sampling, using measurement techniques that are more advanced than possible during continuous monitoring;

- In 2016, a total of 79 samples were collected and analyzed from the first 23 ships with EGCS.

Sampling continued and in 2018 a re-analysis was performed on a total of 281 samples taken from 53 vessels.

\section{SAMPLING AND METHODS OF} ANALYSIS

\subsection{Sampling of probes:}

Samples shall be collected and analyzed in accordance with a standard protocol developed in cooperation with laboratories. The samples are taken by the environmental officers and the ship's engineers, following a training program on board. The basic data for the test include values for EGCS tower input / output.

The extended sample database also includes samples from several vessels with filtration with wash water installed, providing a fourth sample from a point before this filter.

\subsection{The analysis:}

The analysis of the samples is performed by ISO 17025 certified laboratories using approved methods (or equivalent) to test 54 parameters, including PAHs and metals.

A "net post-EGCS" methodology is used to compare with different water quality standards, with the additional analytical step of using a low average, excluding statistical aberrant values more than three standard deviations from the average.

\section{COMPARISON WITH WATER QUALITY STANDARDS \\ - Sample analysis shows average levels of PAH and nitrates below IMO washing water criteria \\ - Average crude PAH and nitrate concentrations as\% of IMO criteria}


- Flush water discharge limitations vary depending on the EGCS wash water flow rate, ie the higher the wash water flow rate, the lower the allowable concentration.

- Here the weighted average post-EGCS gross concentrations are compared with the lowest theoretical requirements.

- Both post-EGCS PAH values for phenanthrene and the mean sum of all detected PAH values are below the strictest IMO requirements.

This comparison is for illustrative purposes only and does not constitute the approval of any ship with IMO requirements (Table 1)[1].

Table 1. IMO requirements for PAH and nitrate

\begin{tabular}{|c|c|c|}
\hline Compund & $\begin{array}{c}\text { Lowest IMO } \\
\text { criteria (based } \\
\text { on a flow rate } \\
\text { of } 90 \mathrm{t} / \mathrm{MWh})\end{array}$ & $\begin{array}{c}\text { Average gross } \\
\text { concentration } \\
\text { after EGCS }\end{array}$ \\
\hline PAH & $25 \mu \mathrm{g} / \mathrm{L}$ & $1.77 \mu \mathrm{g} / \mathrm{L}$ \\
\hline Nitrate & $30 \mathrm{mg} / \mathrm{L}$ & $0.8 \mathrm{mg} / \mathrm{L}$ \\
\hline
\end{tabular}

\section{WASHING WATER EVALUATION}

Comparison of wash water with different water standards is not intended to indicate compliance with them - only to illustrate the quality in an easy to understand way. The comparison is made considering the criteria of several standards:

- German Waste Water Ordinance: Limitations on point sources of wastewater from bio-waste treatment

- EU surface water standards part of the EU Water Framework Directive; a water quality standard showing the maximum permissible concentration in inland surface waters.

- This method uses the difference between the mean of the input values and the mean of the post-EGCS values (sampling point 2 - point 1 ).

- All undetected sampling parameters receive a value equal to $50 \%$ of the detection limit of laboratory equipment.

The results presented exclude statistical values greater than three standard deviations $(3 \sigma)$ from the mean value of the sample parameter (Figure 1) [1],[2], [3], [4], [5], [6].

- Comparing the wash water with different water standards is not intended to indicate compliance with them - just to illustrate the quality in an easy way to underestand.
- The study and comparison with water quality standards does not constitute an environmental impact assessment.

- The aim of this study was to present a snapshot of average wash water concentrations. It was not within the scope of this assessment to identify the relationships or correlations between the concentration of parameters in the wash water and the following factors:

- type and quality of fuel

- fuel consumption

- dilution rates

- seawater inlet flows, EGCS tower and overboard discharge

- sampling motor loads.

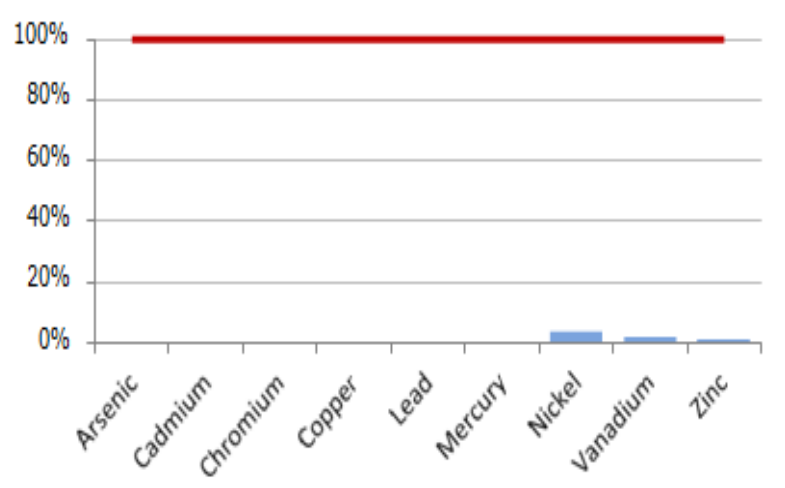

\section{EU Surface Water Standards}

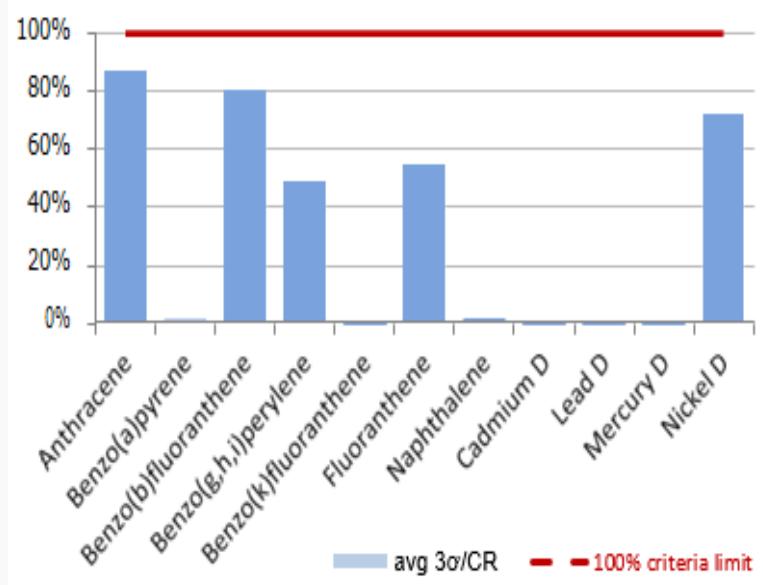

Figure 1 German Wastewater Ordinance and EU standards [1]

The results of the laboratory tests were taken at the nominal value, presumed to be in accordance with the relevant laboratory standards.

Samples are collected and analyzed accordingly (Clean Water Act). Sample kits are prepared and 
provided, each kit containing pre-labeled sample bottles, a sample collection checklist.

\section{CRITERIA FOR EGCS WASH WATER}

Samples were collected by shipboard personnel using a standard training and instruction protocol and analyzed for 54 test parameters at accredited environmental independent laboratories, all using standard methods.

Maritime advisory services were not involved in the sampling process or laboratory analysis, but were hired by the company to:

- compiling and reviewing laboratory test data;

- comparison of wash water test parameters with different water quality standards;

- As there are no water quality standards containing criteria for all parameters tested in the sampling campaign, two terrestrial standards for point source discharge were chosen, in addition to the BMI requirements for EGCS wash water to provide a point reference for total metal concentrations;

- A comparison was also made with water quality standards as a means of assessing PAH levels in context. While water quality standards refer to water bodies rather than a discharge, they illustrate the overall quality of the wash water discharge and offer a broader perspective than comparison with IMO requirements, or simply a compilation of the concentration. media can provide alone.

Unweighted averages of the net post-EGCS concentration were calculated for each parameter and compared with the limits of the relevant criteria for these water quality standards.

The $\mathrm{pH}$ of the point of unloading must be the value that will give at least $6,5 \mathrm{pH}$ four meters from the point of unloading, while the ship is stationary. All $\mathrm{pH}$ values sampled at discharge are above the calculated minimum $\mathrm{pH}$.

The turbidity shall not exceed 25 FNU (formazine neflometric units) or 25 NTU (turflite neflometric units) or equivalent units, above the turbidity of the inlet seawater.

Laboratories analyze all samples using approved methods and issue a final report with all calibrations, standardization and quantification limits.Upon receipt in the laboratory, a checklist is created to document the condition of the sample sets, including temperature, rupture, custody seals and confirmation that all samples are accounted for.
The samples are analyzed during the waiting time required by the standard method.

The results provided by the laboratories include the analysis methodology used and the relevant calibration and standardization procedures (Figure 2)[7].

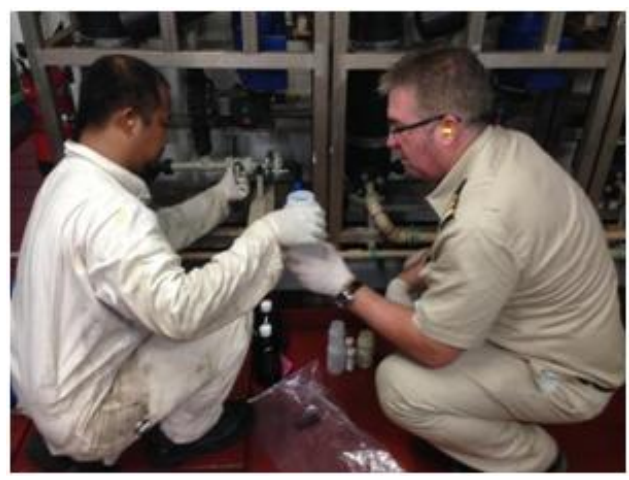

Figure 2 Sampling and analysis [7]

The samples are analyzed during the waiting time required by the standard method (Figure 3 ).

\begin{tabular}{|c|c|c|}
\hline PAHS & Metals* & Other parameters \\
\hline 1. Meithatalen & Aluminu (Al) & C10 - C4OHydrocerbons \\
\hline 2- Methlhatalen & Cadmiu (Cd) & Chloride \\
\hline Acenaphthene & Chrom (Cr) & Total Dissolved Solids \\
\hline Acenaphthylene & Cupru (Cu) & Total Suspended Solids \\
\hline Benzoantracen & Otel $(\mathrm{Fe})$ & AmmoniaasN \\
\hline Benzopiren & Plumb (Pb) & Total Poosphate as PO4 \\
\hline Benzofluoratten & Nickel (Ni) & Total Phosphorus as P \\
\hline Benzoperylene & Thallium (TI) & Total Organic Carbon \\
\hline Benzofluoranten & Vanadiu (V) & Total Kjeldahl Nitrogen \\
\hline Crizena & $\operatorname{Zinc}(Z n)$ & Chromium(VI) \\
\hline Dibenzantracen & Arsenic (As) & pH \\
\hline Fluoranthene & Seleniu (Se) & Nitrate + Nitrate as N \\
\hline Fluorene & Mercur $(H g)$ & Biologica oxygen demand ( 800 \\
\hline $\begin{array}{r}\text { Inderopiren } \\
\text { Naftalină }\end{array}$ & & Chemical oxygend demand (COD) \\
\hline $\begin{array}{l}\text { Nardalind } \\
\text { Phenanthrenene }\end{array}$ & & Total Nitrogen \\
\hline Piren & & Ammonium \\
\hline & & \\
\hline
\end{tabular}

Figure 3 Parameters analyzed

A significant number of samples for each parameter are "undetected" below the limits of laboratory detection. In environmental testing, it is technically impossible to verify the complete absence of an analyte (Figure 4) [2].

If the analyte is not present or present at a concentration below the detection limit, the laboratory will report a non-detect (ND). 
- An analyte is reliably reported as present only when the test result exceeds the detection limit. For the results reported as ND, it was assumed that the analyte concentration in the sample was half the detection limit (DL).

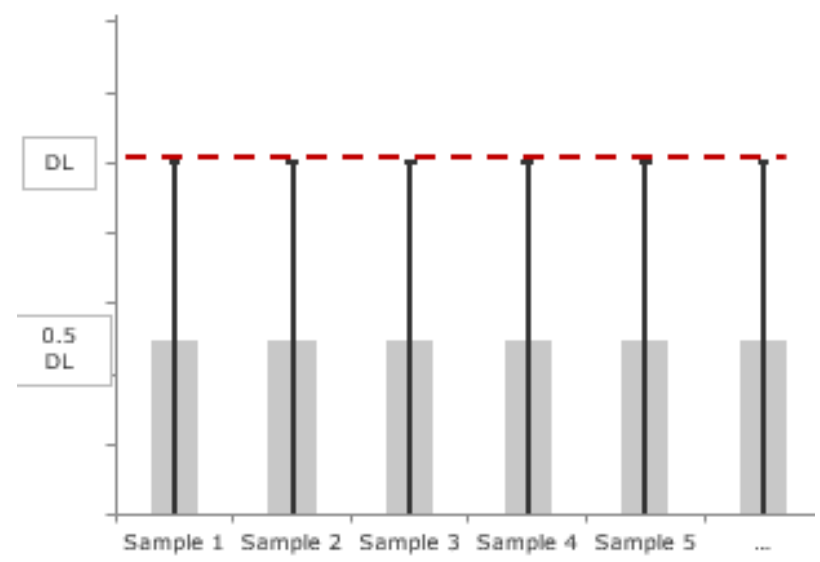

Figure 4 Samples with undetectable ND

DL is provided for each analyte and sample. This means that the analyte concentration is most likely swollen when calculating the average concentration because an ND can actually be zero, while from a chemical analytical point of view, it cannot be definitively identified as zero.

- Certain parameters are almost always below the detection limit at the post-tower output sample.

- An ND is considered to be half of the DL, so the result has more uncertainty, although the margin of error is below the DL.

- For a given parameter, the lower the detection rate, the more likely the average concentration is below DL, but the higher the margin of error.

\section{RESULTS}

Upon receipt in the laboratory, a checklist is created to document the condition of the sample sets, including temperature, rupture, custody seals and confirmation that all samples are accounted for.

\section{concentrations \\ - Calculation of "net post-EGCS"}

- If the seawater in the environment already has a high concentration of the parameter in question, the concentration after the EGCS tower (and in the discharge overboard) may be increased, but not necessarily due to the EGCS process.

- To understand how the EGCS process affects water quality *, a distinction is made between the post-EGCS crude concentration and the postEGCS net concentration (Figure 5).
Post - EGCS concentration $=$ pre-EGCS concentration

(2) - Received seawater concentration (1)
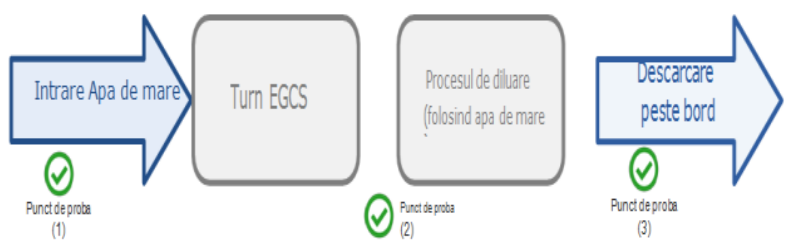

Figure 5 EGCS concentrations

In some cases, the net concentration is negative. This negative result is taken at face value, which is assumed to be valid because there is a seawater filter between the seawater inlet and the EGCS tower.

The intention of using the net concentration is to correct the amount already present in the inlet seawater, giving a clearer idea of how EGCS contributes to the change of the parameter concentration.

The results obtained show:

- Distribution "detects", "does not detect" and statistical values for input and post-EGCS samples (Figure 6).

- Distributions of intake and post-EGCS samples for anthracene and nickel (Figure 7).

\section{- Positive preliminary results for washing water filtration}

Although not further discussed in this evaluation, the results for 11 samples with discharge filtering were also reviewed. Initial tests and laboratory analysis of the filtered material and post-filtration wash water indicate an effective reduction potential for the parameters (Table 2) [3], [4].

Table 2. Washing water filtration results

\begin{tabular}{|c|c|l|l|}
\hline Parameter & $\begin{array}{c}\text { Effective } \\
\text { filtering }\end{array}$ & Parameter & $\begin{array}{c}\text { Effective } \\
\text { filtering }\end{array}$ \\
\hline Arsenic & $\mathrm{P}$ & Antracen & $\mathrm{P}$ \\
\hline Cadmium & $\mathrm{P}$ & $\begin{array}{l}\text { Benzo (a) } \\
\text { antracen }\end{array}$ & $\mathrm{P}$ \\
\hline Chrom & $\mathrm{P}$ & $\begin{array}{l}\text { Benzo (b) } \\
\text { fluoranten }\end{array}$ & $\mathrm{P}$ \\
\hline Copper & $\mathrm{P}$ & $\begin{array}{l}\text { Benzo (g,h,i) } \\
\text { perilena }\end{array}$ & $\mathrm{P}$ \\
\hline
\end{tabular}




\begin{tabular}{|c|l|l|l|}
\hline Steel & $\mathrm{P}$ & Crizena & $\mathrm{P}$ \\
\hline Lead & $\mathrm{P}$ & $\begin{array}{l}\text { Fluoranthen } \\
\mathrm{e}\end{array}$ & $\mathrm{P}$ \\
\hline Mercury & $\mathrm{P}$ & Fluorene & $\mathrm{P}$ \\
\hline Nickel & $\mathrm{P}$ & Naftalină & $\mathrm{P}$ \\
\hline Vanadium & $\mathrm{P}$ & $\begin{array}{l}\text { Phenanthre } \\
\text { ne }\end{array}$ & $\mathrm{P}$ \\
\hline Zinc & $\mathrm{P}$ & Pyrene & $\mathrm{P}$ \\
\hline
\end{tabular}

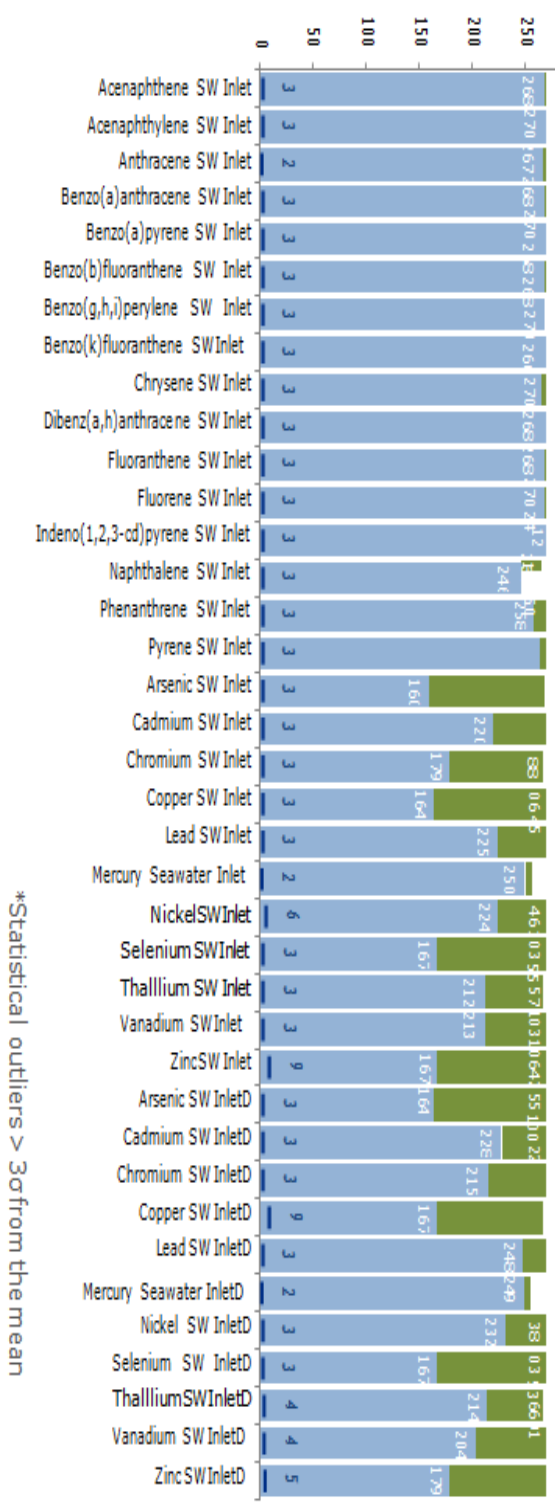

Figure 6 Statistical values for input and post-EGCS tests

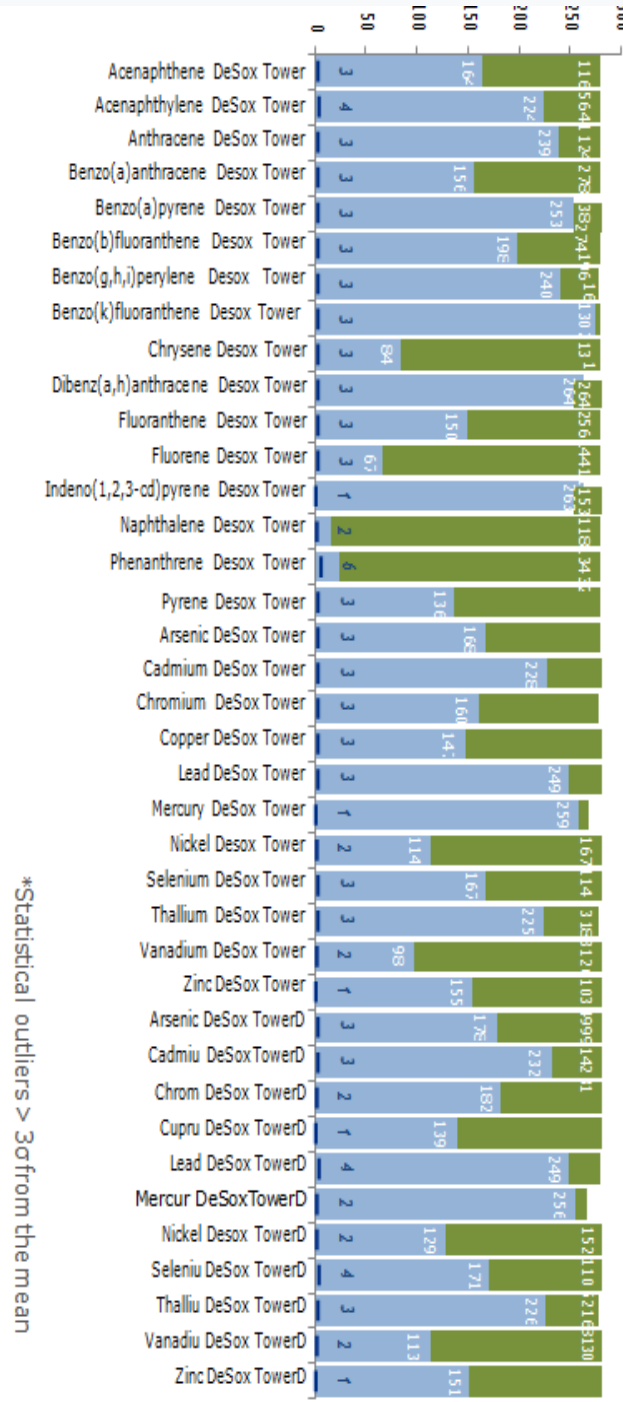

Figure 7 Distributions of intake and post-EGCS samples for anthracene and nickel

IMO encourages regular monitoring to test additional parameters with on-site sampling, using measurement techniques that are more advanced than possible during continuous monitoring [6], [7]. 


\section{CONCLUSIONS}

The following aspects are observed:

- The net post-EGCS average for a number of metallic parameters is extremely low, with post-EGCS output values up to input values: Arsenic, Cadmium, Lead, Mercury, Selenium, Thallium.

- A number of PAH parameters also give a low net result: Benzo (a) pyrene, Benzo (g, h, i) perylene, Benzo (k) fluoranthene.

\section{REFERENCES}

[1] https://www.aquadp.ro/incineratoare/spalare-gaze.

[2]https://www.incineratoare.ro/incineratoare/spalareagazelor

[3] https://inciner.ro/optionale/spalarea-gazelor-emise.

[4]

https://www.aerzen.com/fileadmin/user_upload/02_docu ments/02-02_applications/02-02-

08_marine_industry/A1-050-02__RO.pdf

[5]https://www.alfalaval.ro/industrii/industriaenergetic/motoare-cu-combustie/reducereaemisiilor/reducerea-emisiilor-de-gaz-de-esapament/ [6] Istrate M., 2004, Tehnologii si instalatii pentru reducerea emisiilor poluante, SETIS Publish House, Iasi, Romania.

[7] https://www.youtube.com/watch?v=BIRDUskEnYs 


\title{
COMPARATIVE CFD BASED PARAMETRIC ANALYSIS OF GAS FLOW IN TWO CONTER-FLOW WET SCRUBBER SYSTEMS
}

\author{
Daniela-Elena Jugănaru ${ }^{1}$, Mariana Panaitescu ${ }^{1}$, Liviu-Constantin Stan ${ }^{1}$ \\ ${ }^{1}$ Constanta Maritime University, Faculty of Naval Electro-Mechanics, 104 Mircea cel Batran Street, 900663, Constanta, \\ Romania, e-mail dana.mitu@cmu-edu.eu
}

\begin{abstract}
In this study, gas flow field were simulated using ANSYS 2020R2, Fluid Flow Fluent computational fluids dynamic (CFD) software based on the continuity, velocity, temperature, energy and $\mathrm{k}-\varepsilon$ turbulence. The overall objective of the study is to compare the pressure and velocity maps inside the wet scrubber system for two different scrubber designs. I have choose to analyse an In-Line scrubber bottom inlet, type I, and In-Line scrubber side inlet, type L. Bottom entry or side entry designs to allow a direct up-flow configuration and simplify engine exhaust gas duct routing for any type of vessel. The flow in a wet scrubber is very complex due to the interaction between dust particles, water droplets, and bulk water and flue gas. The flue gas flow is turbulent and there are multiple phases (gas, liquid and solid).

CFD codes that predict gas flow patterns are based on conservation equations for mass, momentum and energy.

The results show that the residuals have a very good job of converging at minimum flow contours and vectors at the inlet across the scrubbing chamber and outlet shows a distributed flow. The velocity profiles have fully conformed to the recommended profile for turbulent flow in pipes.

As can be seen in the figures above, the velocity flow contour inside the scrubber is higher in the scrubber side inlet.

Even though I choose to study the simplified scrubber model without taking into account that spray nozzle improve mixing between the scrubbing liquid and waste gas; from the results obtained, it can be deduced that the numerical simulation using CFD is an effective method to study the flow characteristics of a counter-flow wet scrubber system.
\end{abstract}

Key words : CFD, In-Line scrubber bottom inlet, side inlet, velocity contour.

\section{INTRODUCTION AND LEGISLATIVE FRAMEWORK}

The International Maritime Organization (IMO) has decided that all vessels sailing in the Emission Control Areas (ECA) must reduce sulphur level in fuel oil to $0.1 \%$ or clean the exhaust gas to an equivalent level by 2015. According to Marpol Annex VI are the Baltic Sea, the North Sea, North America (covering designated coastal area in the US and Canada) and the US Caribbean Sea (around Puerto Rico and the US Virgin Island). For the other navigable areas, the sulphur content of the fuel for ships has been limited from January 2020 to a maximum of $0,5 \%$. Until this date, the overall sulphur content limit was $3,5 \%$, a level considered easy to meet for ship operators.

With the implementation of IMO 2020, the International Maritime Organization aims to reduce total sulphur emissions from ships by $77 \%$ from 2020 to
2025. The overall goal is to reduce the negative impact of shipping on human health by reducing air pollution by $68 \%$, especially in the coastal areas of Asia-Pacific, Africa and Latin America.

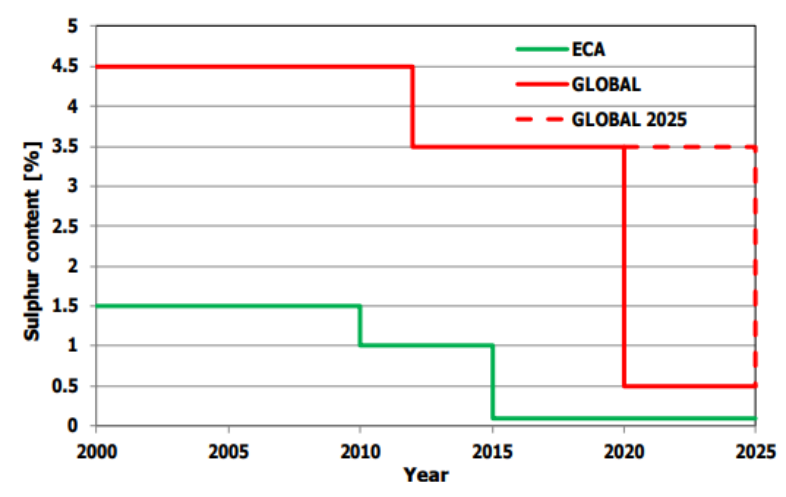

Figure 1 IMO regulation of SOx levels [1] 
Ship operators have the following options to comply with the new sulphur content limits under IMO 2020.

The use of exhaust gas cleaning systems, known as gas scrubbers, is a commercially available option for the transport industry. Vessels equipped with gas scrubbers may continue to burn fuel for boilers with a high gas content and thus may comply with the sulphur limit of $0,5 \%$.

Emission reduction technology works by spraying alkaline water into a vessel to remove sulphur and other unwanted chemicals, through an open loop system, a closed loop system or a hybrid system. The use of gas scrubbers will allow the eradication of almost half of ships' harmful emissions.

\section{OBJECTIVES}

The objectives of the paper are to gain an improved understanding of the processes in wet scrubbers. These flow processes include but are not limited to:

- flue gas and droplet flows;

- agglomeration of droplets;

- flow of bulk water

- Interaction between dust particles and water droplets.

With the improved modelling capability, existing scrubber designs can be simulated with the aim of determining design modifications for improved scrubber performance.

The aims of this study was to simulate the gas flow fields using ANSYS 2020R2, Fluid Flow Fluent computational fluids dynamic (CFD) software based on the continuity, velocity, temperature, energy and $\mathrm{k}-\varepsilon$ turbulence. The overall objective of the study is to compare velocity maps inside the wet scrubber system by vector, contour and streamlines for two different InLine scrubbers, one with bottom inlet designs and other with side-inlet design.

\section{THEORETICAL NOTIONS}

The general approach to the study was to rationalize scrubber performance in terms of basic principles.

Scrubbers are used to control emissions from many diversified sources and are favoured over other air pollution control equipment under the following circumstances:

- Where the removal of gaseous, or simultaneous gaseous and particulate contaminants is required.

- Where the treatment or disposal of the liquid effluent is simple and not expensive.

- Where there is no water shortage.

- Where available plant space is limited.

- Where there is an explosion hazard.
- Where dust resistivity and/or gas temperature is high.

- Where the dust particles are larger than $5 \mu \mathrm{m}$.

- Where the gas is at or above the dew point.

- Above all, where the economics is favourable. [2]

\subsection{Seawater desulfurization system for marine industry}

Using seawater as a desulfurization absorbing liquid, our marine SOx scrubber employs a seawater desulfurization system that has a simple configuration and can be installed in a limited space. The system flow diagram of marine SOx scrubber is presented in figure 1. The seawater taken from the sea is supplied to the absorption tower directly spraying into flue gas. The system utilizes alkaline components naturally contained in seawater to remove sulphur. The marine SOx scrubber complies with the IMO EGCS Guidelines and makes sure that the effluent $\mathrm{pH}$, polycyclic aromatic hydrocarbons (PAHs), turbidity, etc., are within the respective limits before being discharged overboard. Each unit of the marine SOx scrubber needs to be approved by a classification society regarding its compliance with the Guidelines. [3]

IMO allows clean wash water to be discharged overboard in any water area. Clean means:

- turbidity below 25 NTU

- PAH level below 50 ppb

- $\mathrm{pH} 6.5$ (4 meters from ship). $\mathrm{pH}$ can be measured after correction or dilution.

\subsection{Conditions influencing on the design of scrubber system}

In principle, an EGC (exhaust gas cycle) scrubber system can be fitted on any type of ship sailing in any ocean in the word, but a great importance is given to the factors like sea water temperature, sea water alkalinity, fuel oil quality and engine or boiler type that influence on the dimensions and efficiency of the scrubber. There are three big categories of factors, like: external factors, vessel details and legislation.

External factors are fuel oil type and quality, water quality, air temperature and relative humidity, and sea water temperature.

The factors by the vessel details are: engine type and size; space restrictions and limitations on electrical power. Scrubbers are designed to comply with the guidelines as provided by MEPC under IMO, which are being implemented in EU, US and national legislation by taken into account the sulphur limit and discharge water. [4] 


\section{Journal of Marine technology and Environment}

\section{NUMERICAL MODELING}

In this study, gas flow field were simulated using ANSYS 2020R2, Fluid Flow Fluent computational fluids dynamic (CFD) software based on the continuity, velocity, temperature, energy and $\mathrm{k}-\varepsilon$ turbulence. The overall objective of the study is to compare the pressure and velocity maps inside the wet scrubber system for two different scrubber designs. I have choose to analyse an In-Line scrubber bottom inlet, type I, and In-Line scrubber side inlet, type L. Bottom entry or side entry designs to allow a direct up-flow configuration and simplify engine exhaust gas duct routing for any type of vessel.

ANSYS Fluent is arguably the powerful CFD package for simulating multi-phase flows and was chosen for the modelling work in this paper.

The flow in a wet scrubber is very complex due to the interaction between dust particles, water droplets, bulk water and flue gas. The flue gas flow is turbulent and there are multiple phases (gas, liquid and solid).

CFD codes that predict gas flow patterns are based on conservation equations for mass, momentum and energy. [6]

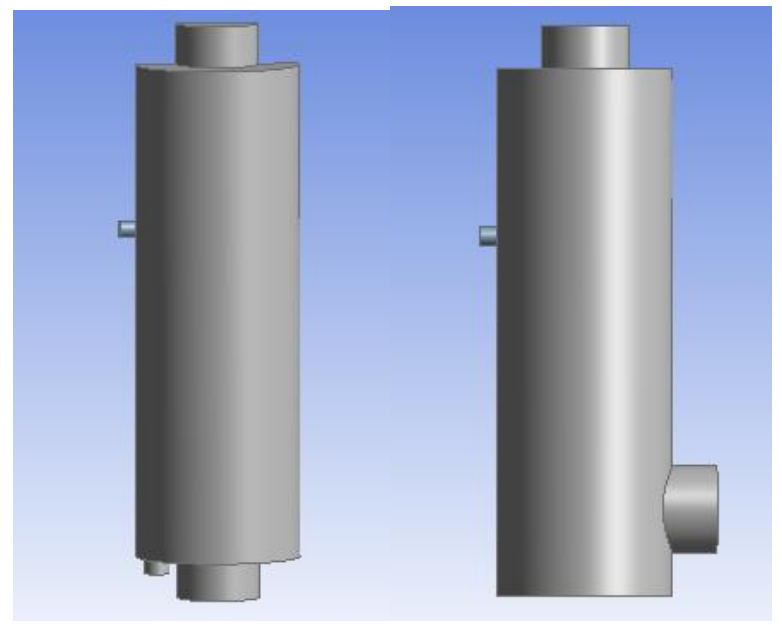

Figure 2 In-Line scrubber bottom inlet and side inlet

Figure 2 shows the three dimensional model of InLine scrubber bottom inlet and In-Line scrubber side inlet. Both models are created in ANSYS 2020R2 and work in Open Loop System.

Table 1 Dimensions of the scrubber analysed

\begin{tabular}{|c|c|}
\hline Part & Value $(\mathrm{cm})$ \\
\hline Scrubber height & 700 \\
\hline
\end{tabular}

\begin{tabular}{|c|c|}
\hline Year 2021, Vol.I & 200 \\
\hline Scrubber diameter & 100 \\
\hline Clean gas outlet diameter & 100 \\
\hline Scrubbing liquid inlet diameter & 20 \\
\hline Slurry outlet diameter & 30 \\
\hline
\end{tabular}

Figure 3 shows a schematic of the mesh used. The mesh near the walls are fine, whereas away from the walls and towards the centre are coarser. A total of 412983 quad elements and 89789 nodes were used in the analysis for the first model and 382531 quad elements and 83148 nodes for the second model.

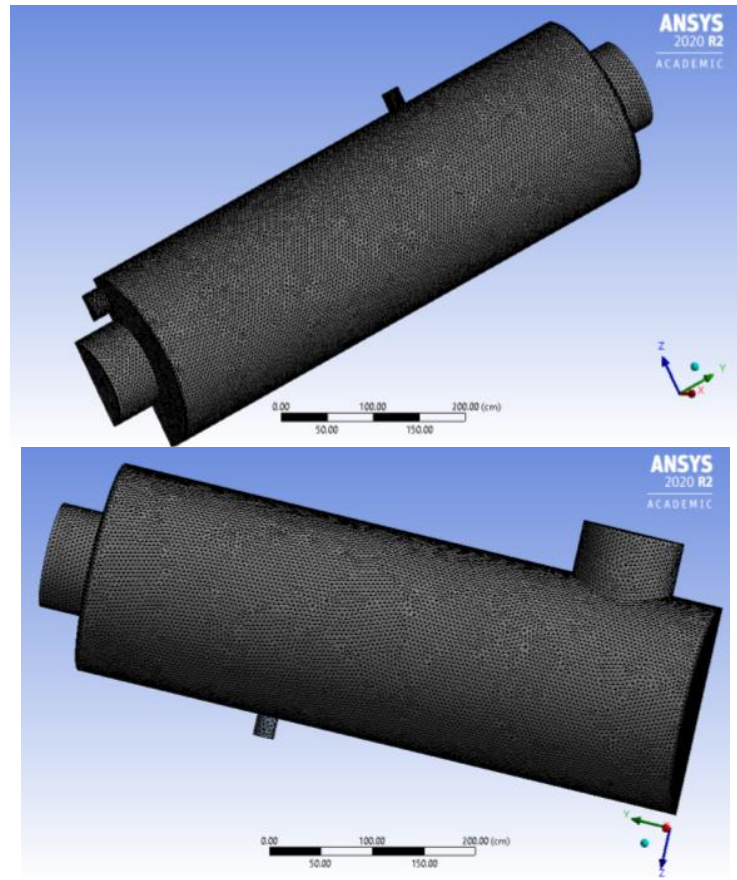

Figure 3 Mesh models

\subsection{Boundary conditions}

Inlet mass flow rate under $100 \%$ engine load was taken $23,72 \mathrm{~kg} / \mathrm{s}$. The pressure for inlet waste gas was $101325 \mathrm{~Pa}$ and the temperature defined was $485 \mathrm{~K}$.

The pressure droplets taken into account in analyse was $1000 \mathrm{~Pa}$ and the temperature was $298 \mathrm{~K}$.

The both models of scrubbers were design with two layers of nozzles inside the scrubber ( 26 nozzles)

The injection pressure of each nozzle is $0,2 \mathrm{MPa}$ and the spray cone angle is $90^{\circ}$. But in this analyse we chose to eliminate all the details for simplified the simulation. 


\section{Journal of Marine technology and Environment Year 2021, Vol.I}

The continuity, velocity, temperature, energy and $\mathrm{k}$ - $\varepsilon$ turbulence for the both cases were represented in the next figure in 50 iterations.

\section{RESULTS}

The results which were analysed are presented below:

- one model with velocity and pressure distributions (Figure 4, Figure 6);

- second model with velocity and pressure distributions (Figure 5, Figure 7.

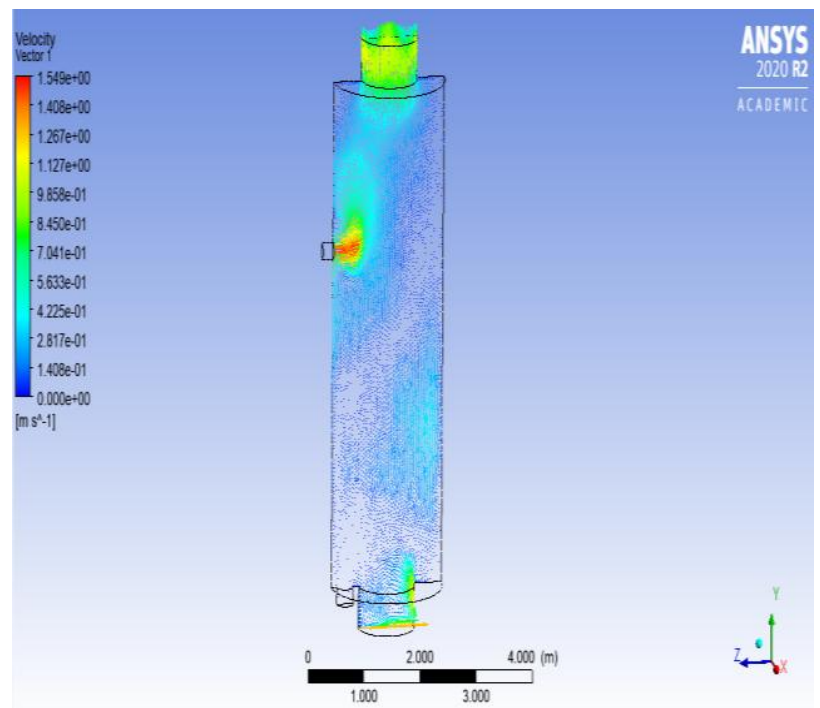

Figure 4 Velocity vector-first model

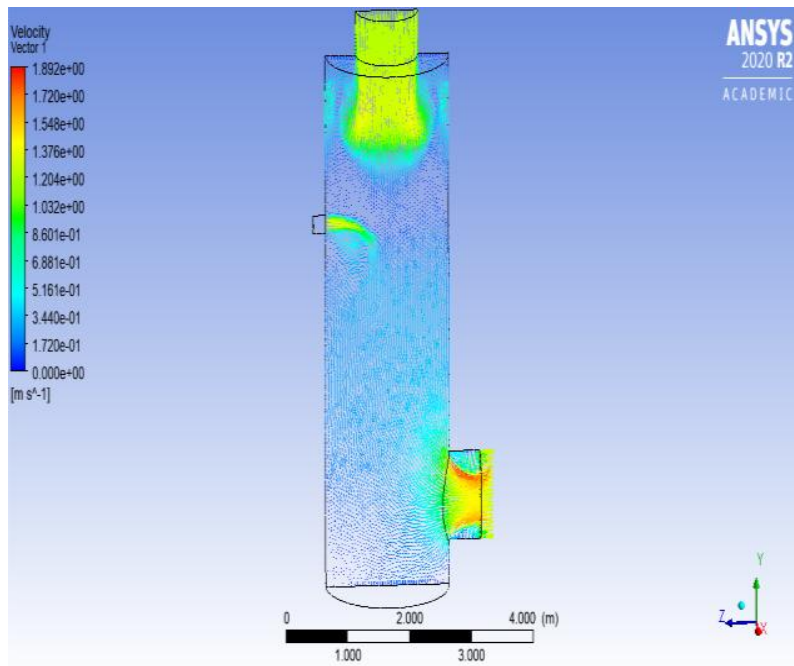

Figure 5 Velocity vector-second model

For the In-Line scrubber bottom inlet, the maximum value of the velocity was $1,5491 \mathrm{~m} / \mathrm{s}$ (Figure 4) and for the other model analysed was $1,892 \mathrm{~m} / \mathrm{s}$. (Figure 5).
For the velocity contour the distributions are presented in Figure 6 and Figure 7 for both models.

For pressure, the minimum value obtain was $183,036 \mathrm{~Pa}$ and the maximum value was $+251,564 \mathrm{~Pa}$ for the In-Line scrubber bottom inlet.

In comparison with this model, for the second one, the values was $-116,021 \mathrm{~Pa}$ and +204,83 $\mathrm{Pa}$.

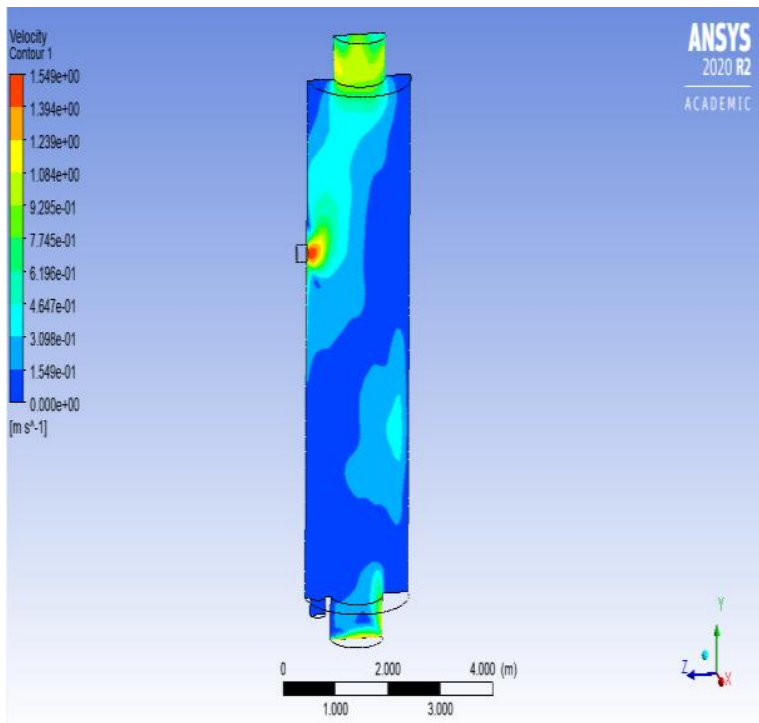

Figure 6 Velocity Contour-first model

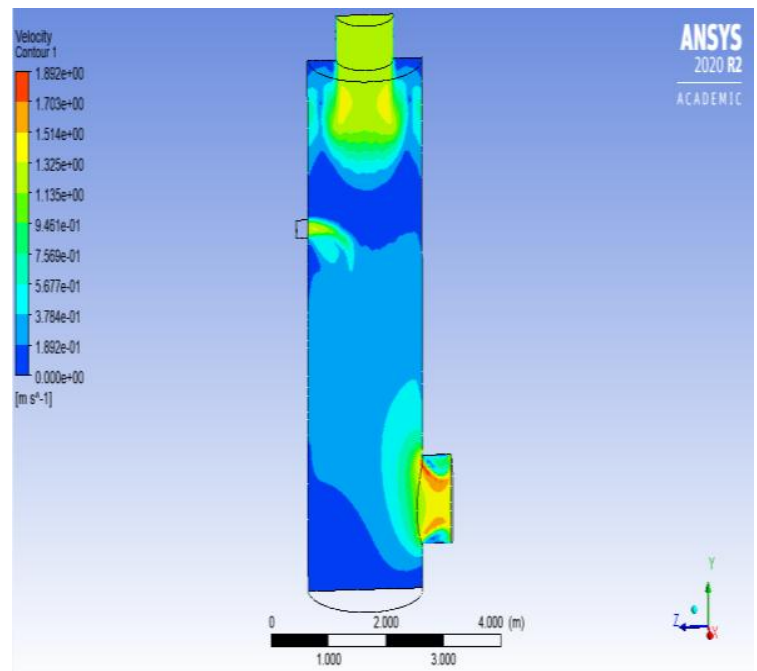

Figure 7 Velocity Contour-second model

The fields of streamline for both models are presented in the Figure 8 and Figure 9. 
Journal of Marine technology and Environment

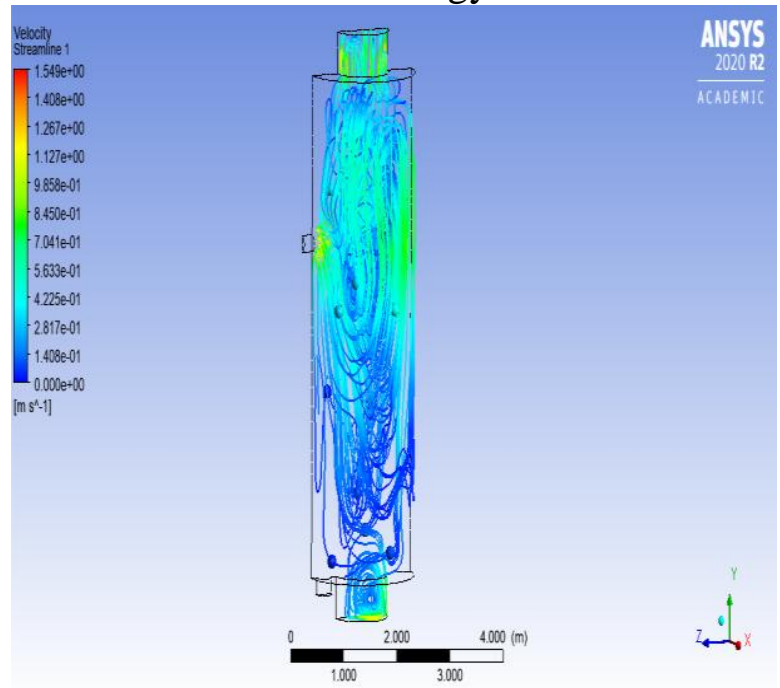

Figure 8 Velocity streamline field model 1

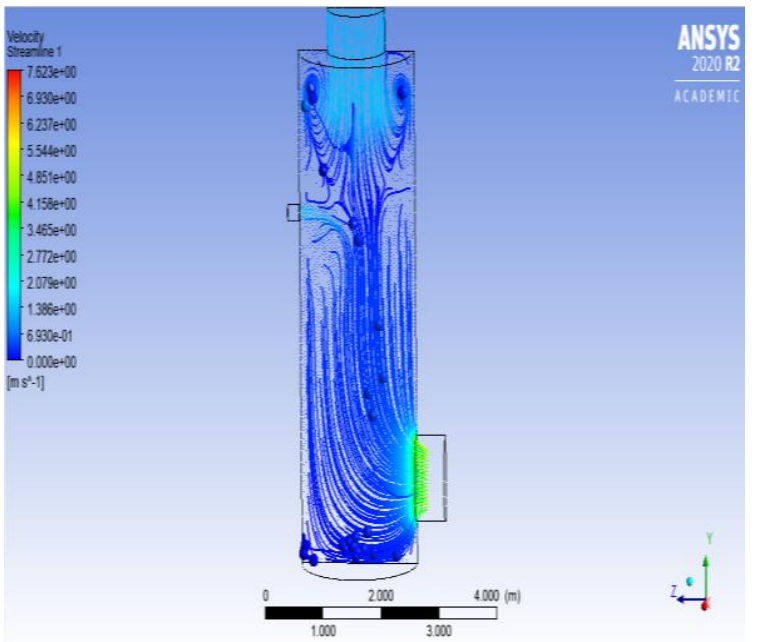

Figure 9 Velocity streamline field model 2

The values for the turbulent kinetic energy $\mathrm{k}$ in the first analyse were between $4,973567 \cdot 10^{-8}$ and 22,106 $\mathrm{m}^{2} / \mathrm{s}^{2}$ and for second analyse were between $3,58187 \cdot 10^{-7}$ and $25,241 \mathrm{~m}^{2} / \mathrm{s}^{2}$.

The total pressure within the scrubber cross-section is constant while the minimum and maximum pressure drops was conformed to the recommended pressure drop for wet scrubber.

\section{CONCLUSIONS}

The results show that the residuals have a very good job of converging at minimum flow contours and vectors at the inlet across the scrubbing chamber and outlet shows a distributed flow. The velocity profiles have fully

\section{Year 2021, Vol.I}

conformed to the recommended profile for turbulent flow in pipes.

As can be seen in the figures above, the velocity flow contour inside the scrubber is higher in the scrubber side inlet.

The difference in velocity values is maintained at about $0,3 \mathrm{~m} / \mathrm{s}$ through the inner surface of the scrubber. The same difference can be observed at the clean gas outlet.

Even though we choose to study the simplified scrubber model without taking into account that spray nozzle improve mixing between the scrubbing liquid and waste gas; from the results obtained, it can be deduced that the numerical simulation using CFD is an effective method to study the flow characteristics of a counterflow wet scrubber system.

\section{REFERENCES}

[1] Green ship of the future. Vessel emission study: Comparison of various abatement technologies to meet emission levels for ECA's, www.greenship.org

[2] Final report and bibliography, Wet scrubber system study, July 1972 Vol II, Prepared for Environmental Protection Agency, Ed. Apt.

[3] Ryozo S., Tatsuto N., et al., September 2019, Practical design of marine SOx scrubber for megacontainer ships, Vol 56, no 3, Mitsubishi Heavy Industries Technical Review.

[4] Hasen J.P., Kaltoft J., et al., 2014, Reduction of SO2, NOX and particulate matter from ships with diesel engines, Environmental Project no 1510.

[5] Mussatti D., Hemmer P., July 2002, Wet scrubbers for particulate matter, Particulate Matter Controls, , EPA/452/B-02-001.

[6] Bird et al., 1960; Roache, 1976)

[7] Stan L., October 2016, Construction testing of an offshore pipeline using numerical methods, Vol 2, Journal of Marine Technology and Environment. 


\title{
TOOLS TO ENGAGE YOUTH IN ENVIRONMENTAL ISSUES DURING COVID-19 PANDEMIC: CONSTANTA MARITIME UNIVERSITY APPROACH
}

\author{
Mirela-Iuliana Sundri ${ }^{1}$ \& Feiza Memet ${ }^{1}$ \\ ${ }^{1}$ Constanta Maritime University, Faculty of Naval Electro-Mechanics, 104 Mircea cel Batran Street, 900663 , \\ Constanta, Romania, e-mail address: iuliana.sundri@cmu-edu.eu
}

\begin{abstract}
Environmental sustainability must be considered as the main target of humanity. To achieve this goal, the activities of many people must change into pro-environmental behaviour. This is often difficult because it involves high costs, giving up some daily habits and getting out of an artificial comfort. In this sense, education has an essential role to play. Young people are the target group for which appropriate methods must be found to help raise awareness of environmental issues and their involvement in solving them. In the current situation, under the restrictions caused by the Covid-19 pandemic, practical applications with large groups of students and observation of the wild environment is difficult to achieve. In November 2020, the Department of Mechanical and Environmental Sciences within the Maritime University of Constanta organized online the activity "S.O.S. Nature" where different environmental processes have been highlighted through simulation programs. The event aimed to increase the awareness of young people about environmental pollution and its consequences, as well as stimulating transversal skills by high school students participating. The interest given by the large participation in this event and the knowledge gained in this activity underlined the importance of modelling and simulation as useful tools through whose comprehensive approach young people can understand complex environmental issues.
\end{abstract}

Key words: environment, integrated approach, pandemic, pro-environmental behaviour, simulation.

\section{INTRODUCTION}

Global environmental changes represent one of the main problems confronting the international community. Environmental sustainability is perhaps the main goal that humanity must consider. Although there has been and is much discussion about the steps that need to be taken at different levels to achieve this goal, there are still many people who do not realize the need to change our behaviour towards actions on nature. Given the complexity of environmental processes, many categories of people ignore imbalances in natural ecosystems because they cannot link cause and effect. In this sense, education has an essential role in promoting proenvironmental behaviour among the broad masses of people and especially among young people. To this end, it is essential to choose the best methods and tools to raise awareness of environmental issues and motivate young people to approach appropriate behaviour [1].

In the local community, Constanta Maritime University it is also recognized as a promotor of environmental awareness among youth. Because of the vulnerability resulted from COVID-19 pandemic reality, the university had to reconsider its environmental awareness actions. Thus, face to face activities were shifted to online activities. This paper describes how technology can be used in order to provide knowledge in social distancing conditions, with optimum results.

\section{PRO-ENVIRONMENTAL BEHAVIOUR}

At the beginning of this millennium we are accustomed to a way of life that we consider comfortable, although with sometimes serious consequences for our health. Locked up in overcrowded cities, we have moved away from nature and we are no longer able to see that nature is suffering. Air, soil or water pollution has the effect of reducing biodiversity and climate change. Environmental problems affect the entire planet, and scientists warn of the worsening of negative effects if radical action is not taken.

However, the measures to be implemented are not always well received by the people. These measures imply a shift in our behaviour toward a proenvironmental behaviour that involves a change in our daily habits [2] [3] [4]; we must try to give up cars and walk more, not to waste water, food, clothes, household items; take care where we store waste. To apply all these measures, we need to feel nature, stay longer in nature and understand nature.

Taking into account the age groups in the population structure, young people are the ones who will 
suffer in the long run following wrong decisions regarding the good management of environmental problems. That is why they should be militant and have pro-environmental behaviour [5]. Unfortunately, many of the young people have moved away from nature, some of them have not left the overcrowded cities for years. They cannot understand environmental issues and do not accept environmental protection measures because they do not have a comparative factor. Many high school students spend much of their time playing various computer games. They do not feel nature. For some of them it is difficult to give up the permanent purchase of clothes and accessories, the return of the old traditional material or the abandonment of disposable packaging.

Engaging youth in solving environmental problems is an important issue for every nation. In order for young people to be aware of the serious environmental problems at global level and to change their behaviour towards sustainability, an important role belongs to the education process [6].

However, young people are very receptive to the new [7]. Given this character trait, they are the part of the population we can focus on first and foremost in a modern approach to environmental issues.

\section{INTEGRATED APPROACH OF ENVIRONMENTAL PROBLEMS}

Environmental processes are complex and difficult to understand, especially for those unfamiliar with this field. To understand them you need in-depth knowledge of chemistry, biology, physics, mathematics, meteorology, etc. In this sense, the models developed and used in various simulation programs that provide an integrated approach to environmental issues and provide an overview of the processes in nature, using data provided from different fields, are of real use. The interdisciplinary and multidisciplinary approach to environmental issues highlights the risks associated with different activities and helps to make the right decisions quickly. With the help of simulations, short or long term forecasts associated with the impact of human activities can be made and in this way the necessary corrections can be highlighted.

Involving youth in environmental issues is a goal for all countries and from this perspective modeling and simulation are extremely useful tools. Simulation programs are modern methods, with which a variety of data can be accessed and countless scenarios can be created. Young people understand natural phenomena through work tools they love, being accustomed to computer games.

\section{CONNECTING YOUNG PEOPLE TO ENVIRONMENTAL ISSUES DURING THE COVID-19 PANDEMIC}

The restrictions imposed by the Covid-19 pandemic and the online educational process have further highlighted the usefulness of models and simulation processes.

On November 27, 2020, the Department of Engineering Sciences in the Mechanical Field and Environment from Constanta Maritime University organized online the activity "S.O.S. Nature". In this activity, with the help of simulation programs, different environmental processes were highlighted. The event aimed to increase the awareness of young people about environmental pollution and its consequences, as well as stimulating transversal skills of high school final classes students.

The event was attended by 61 students from 9 high schools in Constanta and Tulcea counties. The students were assisted online by students of the study program Engineering and Environmental Protection in Industry.

During the "S.O.S. Nature" activity, students were introduced to the HYSPLIT and FoodWeb simulation programs, both of the freeware type, easily accessible by users, from a PC or smartphone, both software with a user-friendly graphical interfaces. The students worked simultaneously with the teacher's demonstration, they became familiar with how to install and run these programs, as well as with the interpretation of the results obtained from these simulations.

HYSPLIT was developed by the US National Oceanic and Atmospheric Administration [8]. This is a model for calculating the dispersion of substances in the atmosphere as well as their deposition. The students simulated a particle pollution. Students became aware of the importance of weather conditions for the speed and movement of pollutant, for atmospheric concentrations of particles (see figures 1 and 2.) and their deposition on the ground (see figure 3). Graphical results in $\mathrm{KMZ}$ format that displays data in the Google Earth browser (see figure 4) or in .gif format, or were very suggestive. 


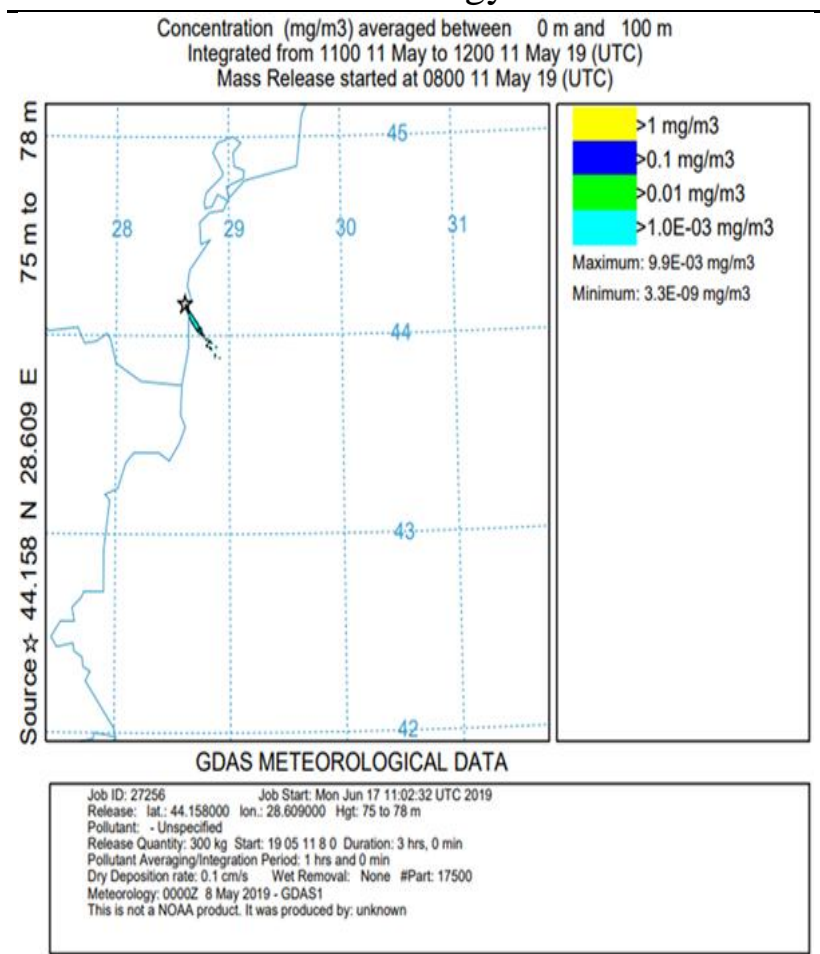

Figure 1. Particle dispersion in the atmosphere - results of the HYSPLIT simulation

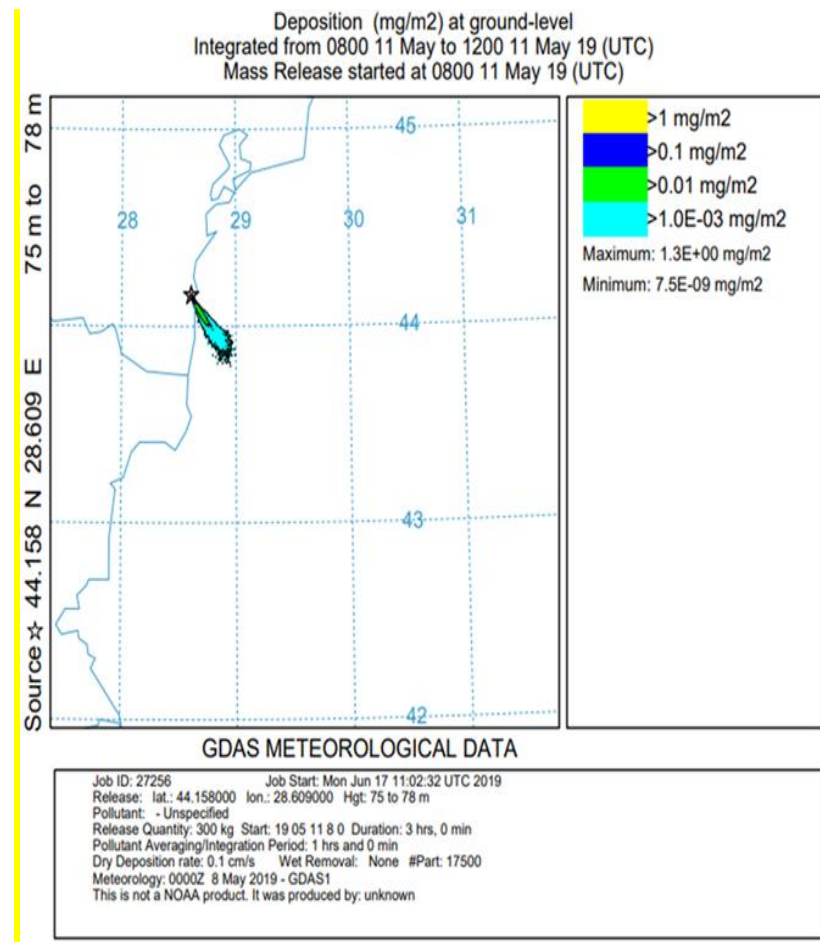

Figure 2. Particle deposition at soil level - results of the HYSPLIT simulation

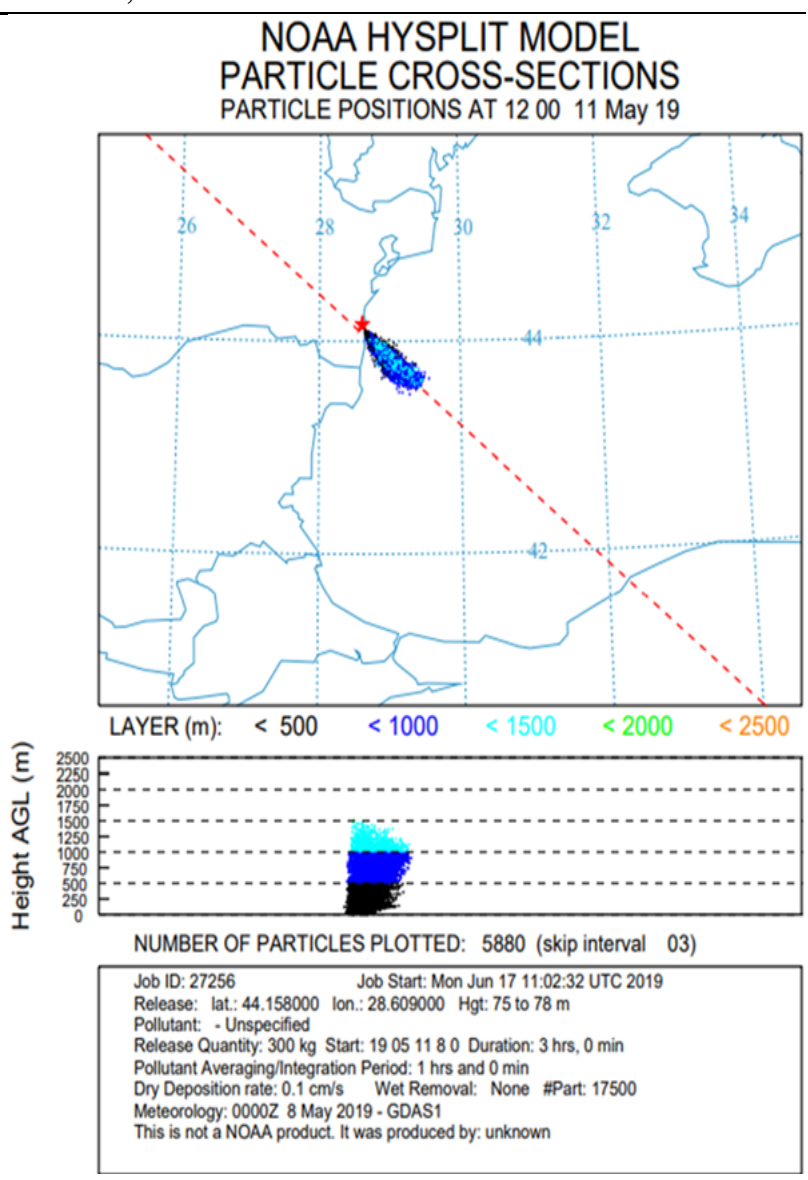

Figure 3. Particle cross-section - results of the HYSPLIT simulation

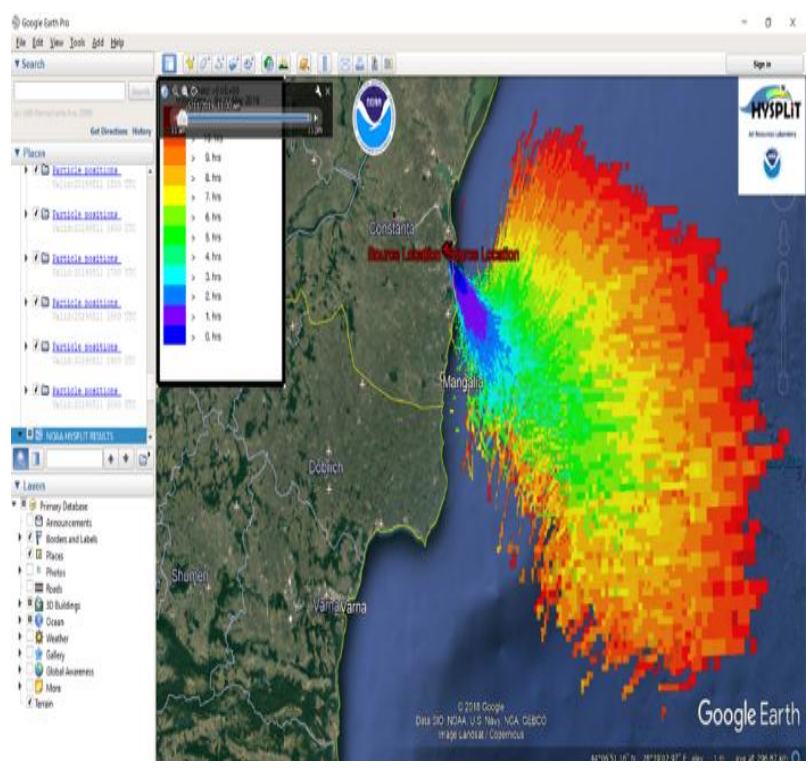

Figure 4. Graphical results of the exercise in the Google Earth browser

The second model used, FoodWeb, was developed by the Canadian Environmental Modelling Center from 
Journal of Marine technology and Environment

Trent University [9]. It is a model of bioaccumulation in complex aquatic systems. With its help, students had the opportunity to track the fate of pollutants along the food chain, to understand that the effects of pollution depend on the type of toxic substance, the abiotic characteristics and the biodiversity of the ecosystem. According to data found in the scientific literature, students designed an aquatic ecosystem with abiotic (see figure 5) and biotic (see figure 6) characteristics, simulating its pollution. Through the simulation program, students received information about the routes of uptake and elimination of a pollutant and highlighted the specific bioaccumulation of the toxicant in various organisms (see figure 7 and figure 8). Students became aware of the importance of simulations to predict the long-term effects of various human activities.

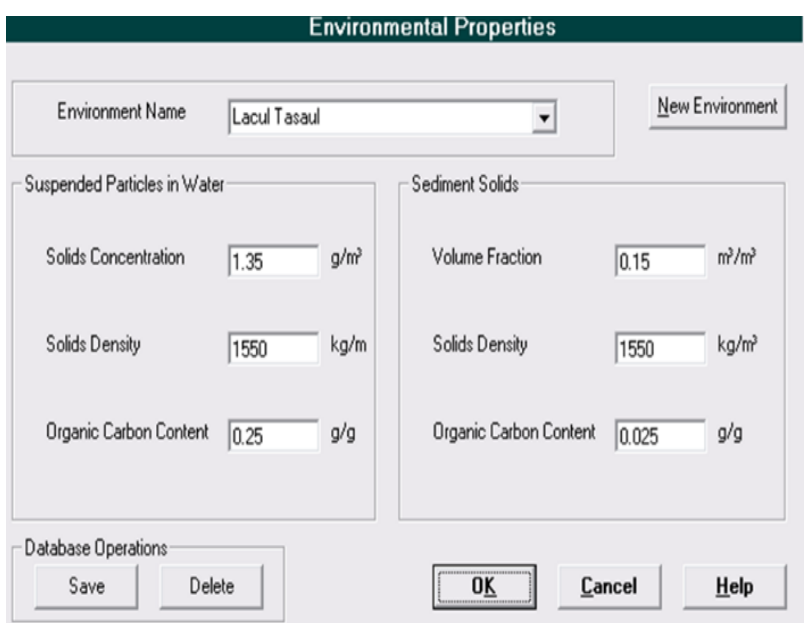

Figure 5. Aquatic environment specific properties

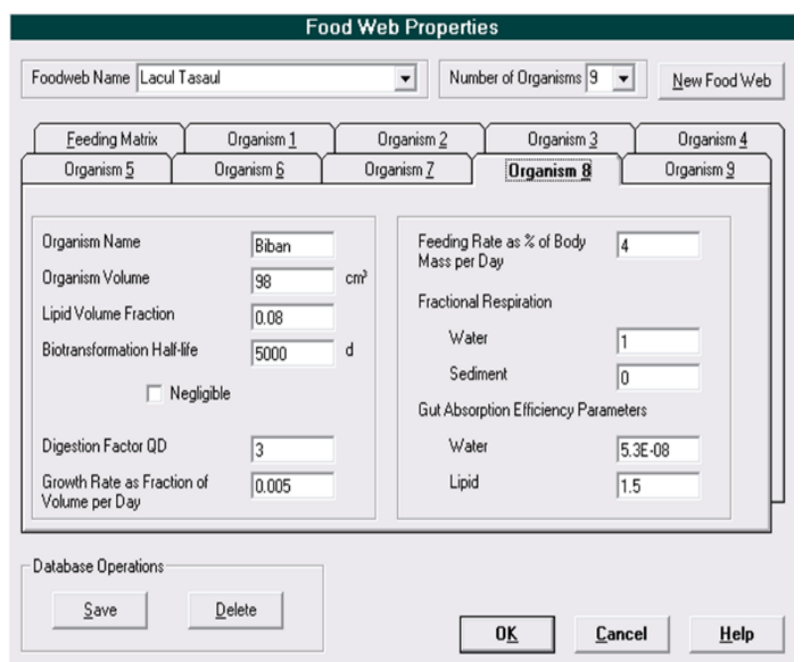

Figure 6. Aquatic Food Web properties
Year 2021, Vol.I

\begin{tabular}{|c|c|c|}
\hline FoodWeb Model & Chemical Name & Beno(s)/grene \\
\hline \multirow[t]{2}{*}{ Vestion 200} & Emvironment Name & LaculTasaul \\
\hline & Foodweb Name & LaCul Tasoul \\
\hline
\end{tabular}

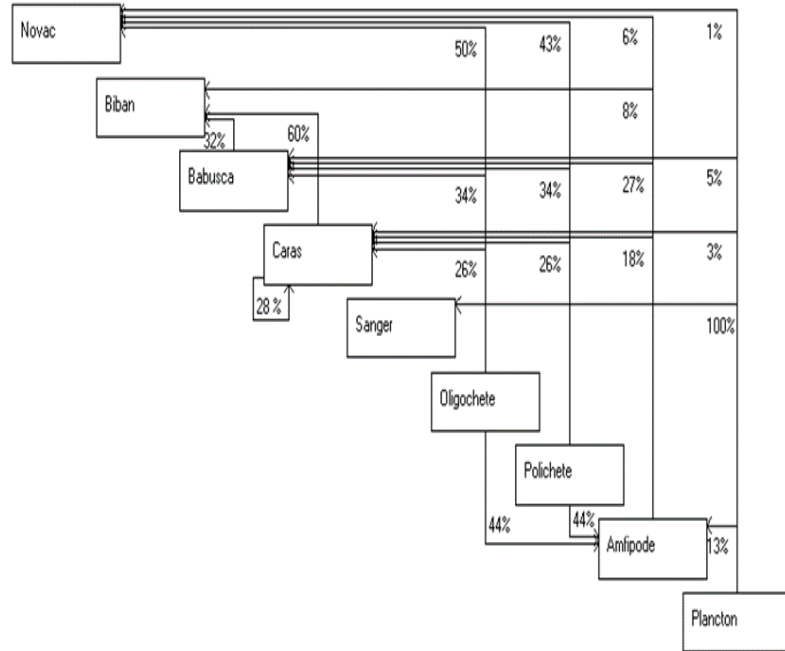

Figure 7. Aquatic specific Food Web Model

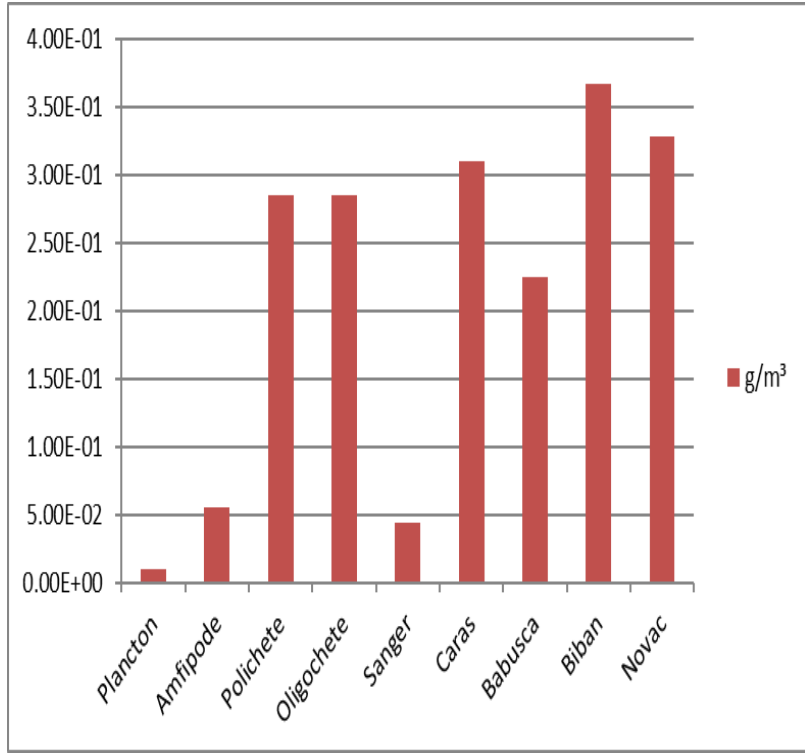

Figure 8. Toxicant bioaccumulation in the food chain

The activity ended with a competition through which the level of accumulation of acquired knowledge was tested. 83 percent of participants answered all the questions correctly. The results obtained demonstrated the interest of young people to understand pollution phenomena and to find ways to solve environmental problems.

\section{CONCLUSIONS}

At international level, environmental education is affected by the pandemic restrictions. Higher education 
Journal of Marine technology and Environment Year 2021, Vol.I

institutions have to adapt to this challenge in a very short time. Constanta Maritime University upgraded its

approach and transfer its attention on the online educational mode, in order to be able to continue its environmental activity with the young people.

Within "S.O.S. Nature" activity, to the students were provided data and exercises to practice interpreting environmental processes. In this way they were able to draw their own conclusions. This increase in awareness of the phenomena that occur around them determines a major involvement in achieving environmental goals by addressing pro-environmental behaviour.

\section{REFERENCES}

[1] Gianluca Grilliab, John Curtisa, 2021, Encouraging pro-environmental behaviours: A review of methods and approaches, Renewable and Sustainable Energy Reviews, Volume 135, January 2021, 110039

[2] Heather Barnes Truelovea Ashley Jade Gillisb, 2018, Perception of pro-environmental behavior, Global Environmental Change, Volume 49, March 2018, Pages 175-185

[3] Florian Lange, Siegfried Dewitte, 2019, Measuring pro-environmental behavior:

Review and recommendations, Journal of Environmental Psychology, Volume 63, June 2019, Pages 92-100

[4] Audra Balunde, Goda Perlaviciute, Linda Steg, 2019, The Relationship Between People's Environmental Considerations and Pro-environmental Behavior in Lithuania, Front. Psychol., 15 October 2019.

[5] Heejin Han, Sang Wuk Ahn, 2020, Youth Mobilization to Stop Global Climate Change: Narratives and Impact, MDPI, Sustainability 2020, 12, 4127; doi:10.3390/su12104127.

[6] [Daniel Thor, Peter Karlsudd, 2020, Teaching and Fostering an Active Environmental Awareness Design, Validation and Planning for Action-Oriented Environmental Education, MDPI, Sustainability 2020, 12, 3209; doi:10.3390/su12083209

[7] Najla Mouchrek, 2018, Engaging College Students in the Transition to Sustainability Through Design-Based Approaches, The Journal of Sustainable Development, Vol. 20, Iss. 1, Pp. 88-103.

[8] https://www.ready.noaa.gov/HYSPLIT.php

[9] https://tuspace.ca/ mparnis/files/Foodweb200.html 


\title{
A POINT OF VIEW ON THE PERCEPTION OF FUTURE PROFESSIONALS ON ENERGY EFFICIENCY OF REFIRGERATION SYSTEMS
}

\author{
Ionela Ticu $^{1}$, Elena Gogu ${ }^{2}$ \\ ${ }^{1}$ Constanta Maritime University, Faculty of Naval Electro-Mechanics, 104 Mircea cel Batran Street, 900663, Constanta, \\ Romania, e-mail address: ionela.ticu@yahoo.com \\ ${ }^{2}$ Gheorghe Duca” Technological High School, Vifor Haiducul Street, No 34, Constanta, Romania, e-mail address: \\ goguelena@yahoo.com
}

\begin{abstract}
In the modern times, energy efficiency is of high interest because there is direct link between this concept and energy conservation, economics, environment and sustainable development. The energy efficiency intensification at international level is closely follow by national leaders and worldwide governments and organisations and by top companies as well. Considering this obvious aspects, higher education institutions are deeply involved in involving energy efficiency in the curricula of future professionals, in order to allow them to gain skills that will help in solving challenges specific to this activity. In this international context, Constanta Maritime University introduced in the curricula of the students enrolled in the specialization called Engineering and Environment Protection in Industry a discipline dealing with this very important activity, named Thermal Efficiency of Buildings and Industrial Processes. This paper is investigating the manner in which our students have mastered the tools of energy efficiency assessment of refrigeration systems, throughout a questionnaire applied to them, at the end of the chapter dedicated to these technologies. The students had to write short comments to very specific questions. Analysis of the comments helps the lecturer and the students to take appropriate measures in the next future. Thus, the feedback resulted to be quite positive because most of the students gained the knowledge provided in this respect. Still, delicate concepts, such as entropy, exergy or exergy destruction seem to raise difficulties to some students. In this respect, results that such an intermediary assessment has to be repeated more often, for other kind of technologies discussed during this course, with the involvement of the concepts introduced by the second law- which are essential in energy efficiency assessments.

Key words: energy, efficiency, thermal, industrial, processes, refrigeration, questionnaire, entropy, exergy, destruction.
\end{abstract}

\section{INTRODUCTION}

In the present times, humanity is facing the fast diminishing of traditional energy resources, the wise use if the existing ones being of capital importance. Since the energy systems are so much present in all our activities, their sustainable operation is an important priority for all scientists. Having in view the new concepts, such as public health, environmental friendly economy and sustainable development, scientists have to approach very carefully the assessment of energy systems- that are issue of continuous innovation in order to reach energy conservation [1]. On the other hand, there is a need in reducing costs specific to the energy consumption, as well. These aspects can be easily achieved throughout rising awareness of future engineers in respect with the management of operations, equipment, devices and energy strategies that will be met during their future activity [2]
In this framework, it is obvious the fact that in technical universities should be implemented adequate educative curriculum materials, able to challenge not only the teaching stuff, but mostly the future professionals, in order to achieve skills, competencies and engineering creativity. Energy Educative Materials have many specific features and lead to important outcomes [3]:

features:

- elevate energy vocabulary;

- understanding of complex situations, concepts, writings;

- continuation of the findings;

- providing of a wider literature survey;

- improved interaction between expert and future professional;

- subtle approach of energy issues;

- easier access to best practices; 
- access to powerful engineering assessment tools; opening to new developments;

- insurance of long lasting and reliable materials. outcomes:

- student facile understanding;

- diminished wrong understanding of fundamentals;

- easier approach of initially difficult seen topics;

- attractive teaching process;

- better integration between theoretical and practical teaching activities;

- boost of research activities;

- closeness to energy issues in a more creative manner;

- easier understanding of energy practices and policies.

Every day life cannot be accepted without refrigeration, technology met in the preservation and transport of perishable or in providing thermal comfort, but which is known to be a large energy consumer [4]. Refrigeration is introduced in the curricula of the discipline entitled Thermal Efficiency of Buildings and Industrial Processes, Year 3, Second Semester, for students enrolled in Engineering and Environment Protection in Industry specialization, in Constanta Maritime University-ROMANIA. In accordance with the above mentioned, within this course treats very seriously the energy efficiency issue, as well. In the time table of the curricula, for these kind of system are allocated $6 \mathrm{~h}$ for theoretical and practice activities. Energy Educative Materials reflecting energy efficiency of refrigeration plants, provided to the students are based on the following pylons [5], [6]:

1. selection of refrigerants: focusing on environmental regulations- expresses by the research on new refrigerants having a zero Ozone Depletion Potential (ODP)and low Global Warming Potential (GWP);

2. selection of the cycle: in the case in which the refrigerants has a high GWP it is needed the adoption of an other cooling system (indirect cooling) with the use of a brine;

3. selection of the working parameters - when it is seen that increase of evaporation and condensation temperatures are affecting the performance of the cycle;

4. adequate thermal insulation of refrigeration rooms and optimum heat balances - based on the fact that limited insulation of the cooled rooms is reflected in the limitation of load capacity, so that it is required the isolation improvement;

5. selection of refrigeration component parts: detailed by compressor selection has to be done so that maximum cooling production is fulfilled at pick needs; it is recommended the adoption of counter flow condenserheat exchanger in which will happen the cooling (in the first quarter), the condensation (in the following two quarters) and sub cooling of the liquid refrigerant; the evaporator has to has fins and needs periodic defrosting;
6. exploitation: are given the steps of the efficient exploitation:

- planning the exploitation;

- respect procedures;

- trained stuff;

- monitories the performance;

- upgraded automation

- update of the plant.

This paper deals with the discussion of a questionnaire submitted to the students mentioned above, in order to have a perspective of the knowledge gained specific to energy efficiency of refrigeration technology. Initially, during the teaching process, students were acquainted with the above written concepts. Also, they have been trained to understand and to use the mathematical formulation of the performance analysis- provided in the following section. The content of the questionnaire and the interpretation of the feedbacks will be analysed in the following.

\section{METHODS AND MATERIALS}

In Figure 1 one can see the cycle of an vapour compression refrigeration system (VCRS) with its main components: evaporator, compressor, condenser, throttling valve; the cycle is the simples one- meaning with no superheating and no sub cooling.

According their curricula, future engineers are trained to know that a viable analysis cannot be obtained only by the use of the first law equations, because exergy analysis allows the assessment of the maximum performance of the system and the finding of the places with exergy destruction. Thus, such a themodynamic analysis should involve formulas specific to the first and second laws.

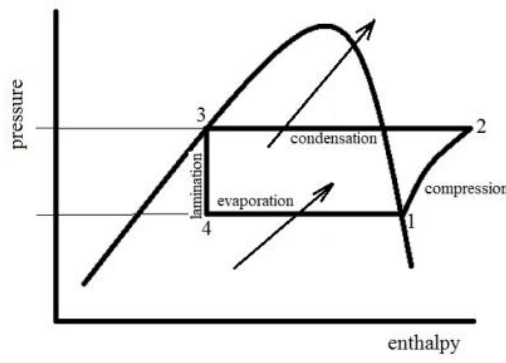

Figure 1 Simpliest cycle of VCRS with specific processes.

For the evaporator are written formulas for the heat load and exergy losses, as given in equations (1) and (2):

$$
Q_{e}=q_{m}\left(i_{1}-i_{4}\right)
$$

here:

$\mathrm{q}_{\mathrm{m}}$ - mass flow, (kg/s) 


$$
\text { Exe }_{e}=q_{m}\left(\text { exe }_{4}-\text { exe }_{1}\right)+Q_{e}\left(1-\frac{T a}{T_{e}}\right)
$$

$(\mathrm{kJ} / \mathrm{kg})$

here:

$$
\begin{aligned}
& \text { exe }- \text { specific exergy, }(\mathrm{kJ} / \mathrm{kg}) \\
& \text { exe }=\left(\mathrm{h}-\mathrm{h}_{\mathrm{a}}\right)-\mathrm{T}_{\mathrm{a}}\left(\mathrm{s}-\mathrm{s}_{\mathrm{a}}\right) \\
& \mathrm{T}_{\mathrm{a}}-\text { surrounding ambiant temperature, }(\mathrm{K}) \\
& \mathrm{T}_{\mathrm{e}}-\text { evaporation temperature, }(\mathrm{K}) \\
& \mathrm{s}-\text { specific entropy, }(\mathrm{kJ} / \mathrm{KgK})
\end{aligned}
$$

The amount of work need to the compressor it is calculated with equation (3):

$$
W_{c}=q_{m}\left(i_{2}-i_{1}\right)
$$

While the exergy losses in the compressor is as given by equation (4):

$$
\text { Exe }_{c}=q_{m}\left(\text { exe }_{1}-\text { exe }_{2}\right)+W_{e l}
$$

here:

$$
\begin{aligned}
& \mathrm{W}_{\mathrm{el}}-\text { compressors electrical power, }(\mathrm{kJ}) \\
& W_{e l}=\frac{W_{c}}{\eta_{m} \cdot \eta_{e l}} \\
& \eta_{\mathrm{m}}-\text { mechanical efficiency } \\
& \eta_{\mathrm{el}}-\text { electrical efficiency }
\end{aligned}
$$

For the condenser, it is possible to write equations for its load- equation (5) and exergy loss formula (6):

$$
\begin{gathered}
Q_{c d}=q_{m}\left(i_{2}-i_{3}\right) \\
\operatorname{Exe}_{c d}=q_{m}\left(\text { exe }_{2}-\text { exe }_{3}\right)-Q_{c d}\left(1-\frac{T_{a}}{T_{c}}\right)
\end{gathered}
$$

here:

$$
\mathrm{T}_{\mathrm{c}}-\text { is the condensation temperature, }(\mathrm{K})
$$

When passing to the throttling valve, the exergy destruction has the form as in equation (7):

$$
\operatorname{Exe}_{T V}=q_{m}\left(\text { exe }_{4}-\text { exe }_{3}\right)
$$

Accorging to the Law 1, the performance is given by the The Coefficient of Performance, written as in equation (8):

$$
C O P=\frac{Q_{e}}{W_{e l}}
$$

While exergy losses, as a whole, are estimated as in equation (9):

$$
\text { Exe }_{0}=E_{\text {Xxe }}+\text { Exe }_{c}+\text { Exe }_{c d}+\text { Exe }_{T V}
$$

As stated, this material will not be limited to the theory of Law 1 of thermodynamics, so that it is given also the exergy efficiency as in equation (10):

$$
\eta_{\text {exe }}=\frac{\text { Exe }_{1}-\text { Exe }_{4}}{W_{e l}}
$$

According to the results from the literature, as seen in [7], [8], when the evaporation temperature increases and the condensation temperature is constant are obtained the following:

- the specific work input increases;

- the Coefficient of Performance increases;

- the specific exergy loss in the compressor increases:

- the specific exergy loss in the throttling valve decreases;

- the specific exergy loss in the condenser decreases;

- the specific exergy loss in the evaporator decreases;

- the exergy efficiency increases.

The energy efficiency questionnaire on refrigeration issues was addressed to 32 students. The aim was to help the lecturer and the students, as well, to find out if this concept is familiar enough to the students, at the end of the chapter dedicated to this technology.

The structure of the questionnaire is given below. The students were asked to write answers in the form of short commnets.

1. In your opinion, which is the most suitable refrigerant to be used in the existing plants?

2. What is the challenge of existing plants in the case of the natural refrigerants use?

3. Which is the most important benefit of the Hydrofluorocarbons (HFCs)?

4. What can you say on the pressures of the refrigerant?

5. What is the effect of evaporation temperature increase on the Coefficient of Performance?

6. Which principle is introducing concepts as entropy and exergy?

7. How can you, briefly, define the entropy?

8. Which is the connection between evaporation temperature increase and irreversibilities?

9. Why increases the exergy efficiency of the refrigeration system together with the increase of the evaporation temperature?

10. Which is the situation in which the plant works at its best energy efficiency?

\section{RESULTS AND DISCUSSIONS}

Question 1: the situation results as follows- $100 \%$ of the students replied that the acceptable refrigerants are the one showing low GWP. The answer is correct, since in the light of Kyoto Protocol, the industry is pushed to adopt eco-friendly refrigerants, such as Hydrocarbons, in order to solve the global warming issue. 
Question 2: $100 \%$ of the students replied that the challenge consist in adapting the plant structure to the properties of the new refrigerant.

Question 3: $100 \%$ of the students replied that HCFs do not contain Chlorine in their formula, this is why they do not affect the ozone layer.

Question 4: refrigerants pressure should allow their work with acceptable sizes for pipes and compressors. $10 \%$ of the students replied considering their working pressures, while $80 \%$ comments stated that the evaporation pressure should be low and the condensation pressure should be high; the rest of the comments stated that there should be a pressure difference between the low and high side of the plant.

Question 5:70\% of the answers say that COP is increasing together with the increase of the evaporation temperature, but only $65 \%$ continue with the explanation: this is due to the fact that the refrigeration effect is increasing; $10 \%$ replied that the pressure ratio decreases, while $20 \%$ didn't answer.

Question 6: $72 \%$ of the answers stated that these two concepts are introduced throughout the second principle pf thermodynamics, $16 \%$ of the comments contain the fact that entropy is introduced by the first law and exergy is introduced by the second law; $9 \%$ included the both parameters in the theory of the first law, while $3 \%$ couldn't answer at all.

Question 7: Entropy is a measure of randomness or disorder within a process. $81 \%$ of the comments say that entropy is a thermodynamic parameter- without any additional specifications, in $11 \%$ cases was seen a confusion between entropy and enthalpy (11\% gave the definition for enthalpy), while $8 \%$ was not able to offer any answer/ comment.

Question 8: The rise of evaporation temperature leads to the irreversibility decrease. $84 \%$ of the students are aware of the fact that real process are irrevesibile, so that their comments refer to the fact that the difference between the ideal cycle and the real one consists in the irreversibilities met in the components. From these, only $30 \%$ continued with the right comment. The rest were not able to comment at all.

Question 9: The increase in of the evaporation temperature leads to decrease of irreversibilities resulting the decrease of exergy losses in the components. For this reason, the exergy efficiency will increase. $90 \%$ of the students replied that if COP increases together with the evaporation temperature increase, it is normal to expect an improvement in the exergy efficiency values. The rest were not able to answer/ comment this question.

Question 10: $95 \%$ of the comments stated that the energy efficiency of the refrigeration plant is connected to the selection of the proper eco- friendly refrigerant, able to provide high values for COP and exergy efficiency, while the rest ignored the exergy efficiency.

\section{CONLUSIONS}

In order to provide on the working market professionals well trained in the field of energy efficiency specific to refrigeration plants, are needed efforts done by the lecturer and the students as well.

The main aspects of this topic- optimised isulation, search of new refrigerants, specific attention on compression systems and cooling systems, have to be rigorously debated during teaching activities. Still, a brief questionnaire submitted to the students at the end of the chapter dedicated to this technology is welcomed, when it is about assessing an intermediary knowledge evaluation.

The questions included in the questionnaire were clearly stated and reflected subjects discussed during theoretical and practical activities.

The comments provided by the students are encouraging, seen the statistics of their comments for each question. Still, there are aspects to be improved. It was seen that comments related to second law theory couldn't achieve a good score. The explanation might consist in the fact that students forgot this theory delivered a year before, or this theory was not well understood at the time when was delivered. On the other hand, it is true that concepts such as entropy, exergy, exergy destruction are quite delicate for students, this is why are needed more efforts in order to clarify them, at any moment.

For this reason, it is indicated to continue with this kind of interrogation as often is possible, by involving this concepts in the assessment of other technologies studied within the discipline Thermal Efficiency of Buildings and Industrial Processes.

\section{REFERENCES}

[1] Atmaca A., Atmaca N., 2018 , Energy efficiency and engineeering applications in conjunction with the International Energy and Engineering Conference 2016, Energ. Econ. Emvorion, Vol 3, No 1, pp 1-4.

[2] Twumasi E., Frimpong E., A., Appiah D., O., Okeyere P., Y., 2017 , Energy efficiency awareness and preparedness among students, IEEE PES-IAS Power Africa, pp 456-461.

[3] Pompea S., M., Walker C., E., 2020, The importance of pedagogical content knowledge in curriculum development for illumination engineering 14 th Conf on Education and Trening and Photonics: ETOP, Proc of SPIE, Vol 10452, pp 104526R-1-104526R-10

[4] Memet F., 2016, Comparative performance analysis of R134 $a$ and R290/R600a refrigerants in a vapor compression refrigeration cycle Journal of Marine technology and Environment, Vol 2, pp 31-34

[5] Hristov H., 2018 , Energy and environmental efficiency of industrial refrigeration installationsalysios, Acta Technica Corviniensis, Bulletin of Enfineering, Tome XI, Fascicule 3, pp 43-44 
Journal of Marine Technology and Environment Year 2021, Vol.1

[6] Ruiz V., 2012 , Analysis of existing refrigeration plants onboard fishing vessels and improvement possibilities Second Int Symposium of Fishing Vessel Energy Efficiency E-Fishing, Vigo, Spain Marine, 8 pp [7] Memet F., 2019, A way to increase safety in marine refrigeration when using ammonia as a working agent CMU Annlas, Year XVIII, Vol 27, pp 37-40

[8] Memet F., 2020 , Energy and exergy analysis of a VCRS working with R600a within a thermodynamkc analysis IOP Conf SE.: Mater.Sci Eng. 916012066, pp 18 


\title{
INSTALLATION OF SUBMARINE CABLES IN THE OFFSHORE WIND INDUSTRY AND THEIR IMPACT ON THE MARINE ENVIRONMENT
}

\author{
Valeriu-Florian VASILESCU ${ }^{1}$, Dumitru DINU ${ }^{2}$ \\ ${ }^{1}$ Constanta Maritime University, Doctoral School of Mechanical Engineering and Mechatronic, 104 Mircea cel Batran \\ Street, 900663, Constanta, Romania, e-mail vasilescuvaleriu75@gmail.com \\ ${ }^{2}$ Constanta Maritime University, Doctoral School of Mechanical Engineering and Mechatronic, 104 Mircea cel Batran \\ Street, 900663, Constanta, Romania, e-mail dumitru.dinu@ gmail.com
}

\begin{abstract}
This article presents the main methods of installing submarine cables used in the offshore wind industry and the impact they have on the marine environment.

From this article, the reader will be able to understand the basic principles that are taken into account from the design phase of a submarine cable, principles that seek to streamline their installation, operation and maintenance and their impact on the marine environment. Given the scale of the development of the wind industry, especially offshore, the length of submarine cables that provide energy transport from the wind farm to shore consumers is also constantly growing. The construction and operation of offshore wind energy systems has been and continues to be regarded with scepticism by environmental activists. Despite the undeniable benefits of this renewable energy source, the impact on the marine environment must also be taken into consideration.

We studied the most efficient methods of installing submarine cables in the offshore wind industry - study which also includes analysing the behaviour of submarine cables and analysing ships' movement during cable transport and installation.

This article is only part of a major research on the installation of submarine cables in the offshore wind industry.

In terms of the frequency and relatively short duration of submarine cable installation operations, on a small strip of up to $8 \mathrm{~m}$, the disturbances and impact caused by these operations are considered minor and are preferred compared to bottom trawling operations and dredging, which are repetitive and more extensive. A single impact, such as cable burial operations, is preferred to continuous, multiple or recurrent impacts. [1]
\end{abstract}

Key words: wind energy, environment, technologies, analysis, efficiency, marine, cable layer.

\section{INTRODUCTION}

A 2015 Joint Research Center (JRC) report "HVDC Submarine Power Cables in the Word" mentioned that there are almost $8,000 \mathrm{~km}$ of highvoltage, direct current (HVDC) submarine electrical cables in the world. However, considering the current pace of offshore wind farms construction, submarine cables have become a ubiquitous element in electricity transmission.

Over $70 \%$ of the world's HVDC submarine cables (both in terms of number and length) are located in the seas adjacent to Europe (Fig. 1).

The first commercial HVDC submarine cable was installed in 1954 in Sweden by ABB Ltd., connecting the island of Gotland and the mainland. It had a length of 90 $\mathrm{km}$, a voltage of $100 \mathrm{kV}$ and a capacity of $20 \mathrm{MW}$. Since then, the technology has evolved towards a higher transport capacity and voltages.

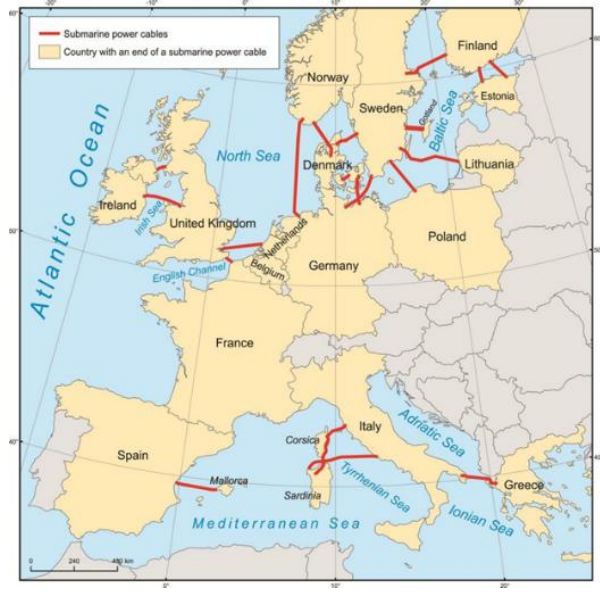

Figure 1 Submarine power cables in Europe [2] 


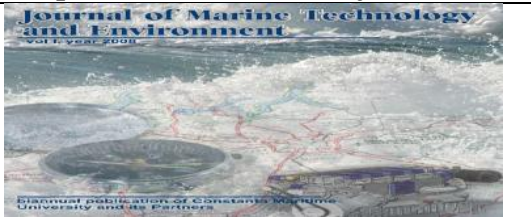

\section{DETERMINANTS IN THE MANUFACTURE AND INSTALLATION OF SUBMARINE CABLES}

\subsection{Geological study and survey of the cable's installation route}

A mandatory geological study of the cable route must be carried out before installing the submarine cable. This study should provide a complete and complex picture of the seabed in terms of bathymetry, depth gradient, nature of the seabed, environmental conditions. All these investigations are performed and evaluated by geophysicists, geologists, oceanographers using dedicated equipment. An incorrect assessment of these conditions would lead to improper cable design and therefore to malfunction or additional maintenance costs.

\subsection{Geological structure}

Knowing the nature of the seabed is of critical importance. Placing submarine cables in stable environments will extend their life and guarantee an easier operation. The nature of the seabed is very diverse due to its varied geology and the processes that affect it.

Most of the seabed is covered by a thick layer of sediment that averages $450 \mathrm{~m}$ (Fig. 2). This layer can be thinner on the ridges in the middle of the ocean, where the structures, formed by hard rock and sometimes even volcanic lava flows, stand out.

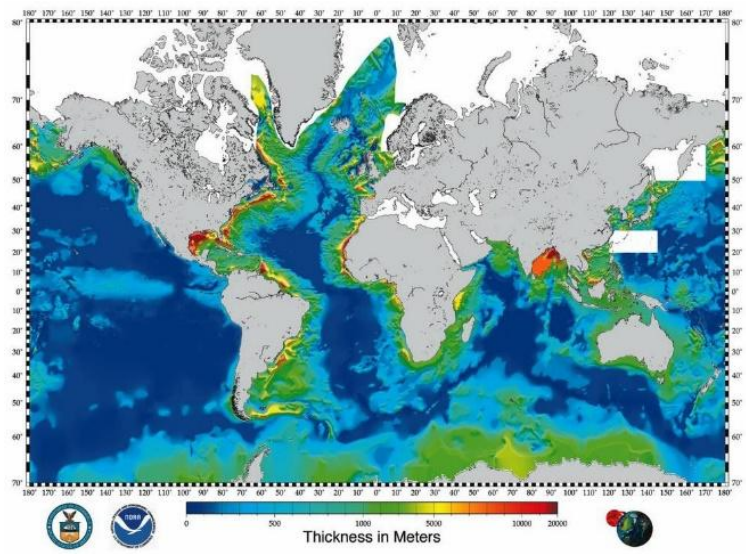

Figure 2 Total sediment thickness of the world's oceans and marginal seas [3]

Although the predominant process below the water surface is sedimentation, the movement of water can move finer particles, especially in coastal areas, causing changes in bathymetry on the seabed. In soft sediment (clay, mud, earth, sand), the easiest solution is to place the cable in a dug ditch. However, this also means that the cable can be easily removed or moved by waves and currents, which can damage it. This risk decreases with increasing depth, where the action of waves and sea currents decreases or becomes negligible. Harder sediments, such as gravel, provide better protection because they are heavier and less prone to movement, but harder to dig and therefore lead to higher costs.

\subsection{Water depth and subsea bed topography}

When choosing the routes for installing the cables, try to avoid deep trenches or steep slopes, while maintaining the shortest possible path. Because most submarine electrical cables installed so far cross shallow, flat-bottomed seas covered with thick Quaternary sediments (Baltic Sea, North Sea, Irish Sea, English Channel, Strait between Japan, Philippines, USA, Canada, Australia, New Zealand) the depth of the water and the slope were not a major concern. The depth threshold of $1000 \mathrm{~m}$ was exceeded only in the Mediterranean Sea.

In water environments, special attention must be paid to the hydrostatic pressure exerted by the water column, which could become an important factor both in the design and choice of materials used and in the methods of cable installation or repair. The pressure increases constantly with depth, with about one atmosphere (1 atm) every $10 \mathrm{~m}$.

The submarine cables installed so far cross the continental seas. The continental shelf is characterized by shallow waters (up to $200 \mathrm{~m}$, in some cases up to 400 $\mathrm{m})$ the depth increasing slightly, on average with an angle of $0.1^{\circ}$ and occasionally reaches $1^{\circ}$ (Fig. 3).

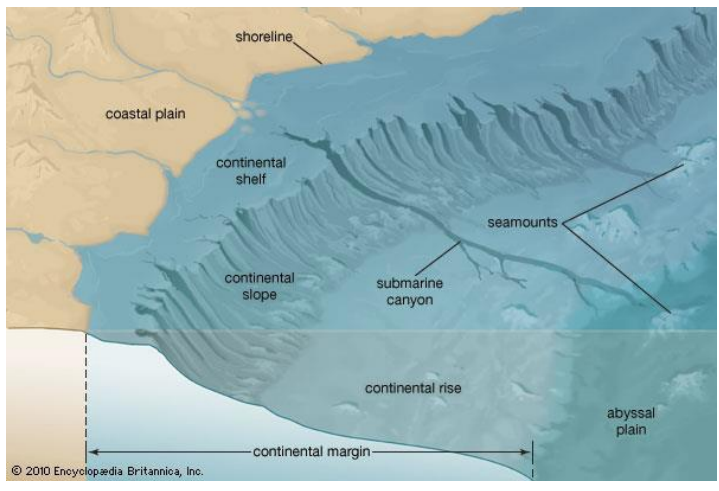

Figure 3 The main components and major submarine landforms of a continental margin) [4]

\subsection{Geodynamic processes}

On continental shelves, where the value of the slope gradient is higher, the deposition in large quantities of fine sediments can raise problems of slope stability. When combined with coarser sediments brought by rivers and streams from the mainland, their balance could become unstable. Water fills all the spaces and pores in the sediment and can act as a lubricant. An event such as an earthquake can trigger a mass of sediment. Sometimes, the own weight of the sediment can increase beyond the stability threshold, and the 
landslide can occur without the occurrence of such events.

From the investigations of the causes that led to the interruption, over time, of several submarine telecommunication cables, information was obtained about the geodynamic processes that affect the submarine sediments [5].

In areas close to the shore, the main dynamic processes are determined by waves and sea currents, as well as by the flow of sediment discharged by rivers. The magnitude of their actions depends on the local topography of the coast, the depth of the water and the climate pattern (precipitation, humidity, etc.). In areas with high seasonal rainfall and a soft soil structure (sand, soft sandstone, clay) erosion can be strong enough to turn large amounts of soil into sediments, which then reach the sea and form sediment deposits. This large amount of material transported over hundreds or thousands of years creates the largest accumulations of sediment on earth. Large sediment accumulations can lose their stability and begin to move along the slope at high speeds that can reach $19 \mathrm{~m} / \mathrm{s}$. The small amounts of sediment encountered at the ends of the canyon should not be neglected either, which in turn can start moving at speeds that can reach $5 \mathrm{~m} / \mathrm{s}$ over distances of hundreds of kilometres [5]. If electrical or telecommunication cables are installed in the direction of these movements, they may be broken or subjected to additional mechanical stress.

\subsection{Waves and sea currents}

Both types of movements can affect submarine cables by the forces developed by the action of water or by redistribution of sediments.

The action of the waves is stronger on the shore or in shallow waters and decreases with increasing depth. In general, the action of the waves stops at a depth of about $30 \mathrm{~m}$. Only exceptionally, during strong storms the action of the waves is felt more deeply. Coastal areas, especially sandy ones, are undergoing the most dramatic changes due to the action of waves. When cables in such areas are not buried deep enough, they can be brought to the surface (Fig. 4)

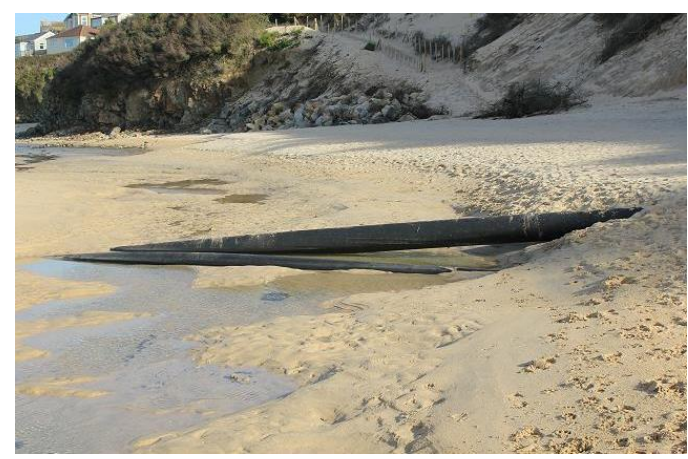

Figure 4 Cable exposure due to waves' action on a sandy beach in Great Britain [6]

\subsection{Temperature, salinity and corrosion}

The characteristics of sea water differ from one place to another due to climatic zones, inland water influences, biotic activity and its depth. The study of these characteristics contributes to the optimal choice of the materials from which the submarine cables are built.

The electric current passing through a conductor causes its temperature to rise. If the current becomes too high, the conductor reaches a critical temperature at which the insulation parts cannot work properly or may even begin to melt. Under normal operating conditions, the cable temperature must not reach these critical limits. Also, the ambient temperature plays an important role in maintaining the functional parameters in their optimal range. Because most cables are located in fairly cold regions, the lower temperature has a cooling effect on the cable, improving its efficiency.

The sea water temperature follows the general pattern of climatic zones with local influences caused by the movement of air masses from the land. As a general rule, the temperature decreases with depth (Fig.5) reaching $4^{\circ} \mathrm{C}$ at $1000 \mathrm{~m}$ depth (Fig.6) maintaining this temperature to the bottom of the sea.

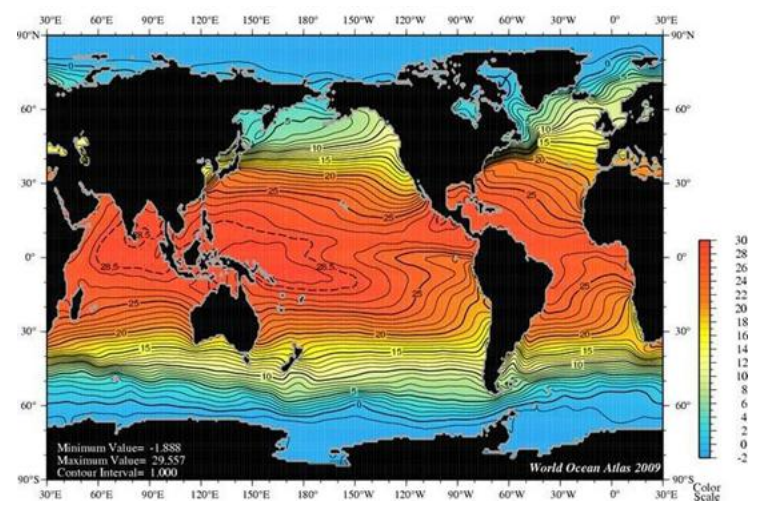

Figure 5 Annual temperature at the ocean's surface [7]

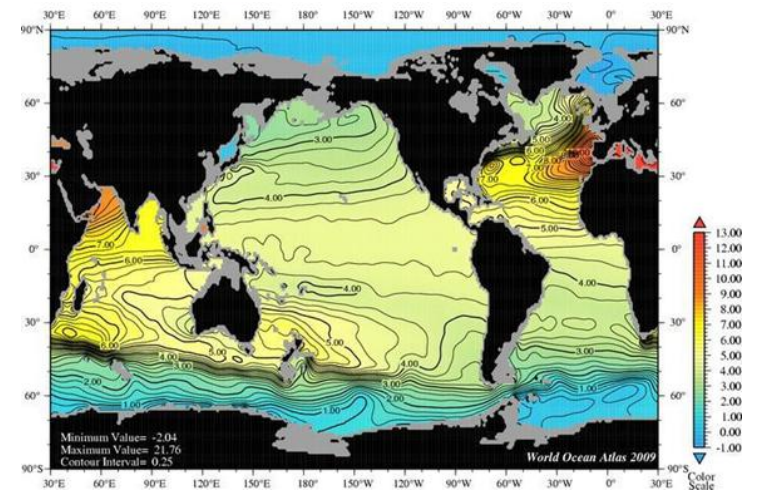

Figure 6 Annual temperature at $1000 \mathrm{~m}$ depth [7]

Salt water is a corrosive environment. Salt is present everywhere in seawater, in varying 
Installing submarine cables is a complex process. In the planning phase, an analysis of this process must be carried out, taking into account factors such as cable properties, route characteristics, available installation equipment and the technical capabilities of the ship intended for installation (Fig.7) shows some of the most influential parameters in an installation operation:

- departure angle is the complimentary angle of the cable exit angle at the overboard chute of the CLV

- top tension is the tension applied to the cable using the onboard cable tensioners

- layback is the horizontal distance between the cable exit point from the CLV and the touch down point (TDP) on the seabed

- bending radius at TDP is the current radius of curvature of the cable and is one of the most important design parameters for cable integrity

- the cable tension on the seabed is the residual tension at TDP, representing another critical parameter for the cable life.

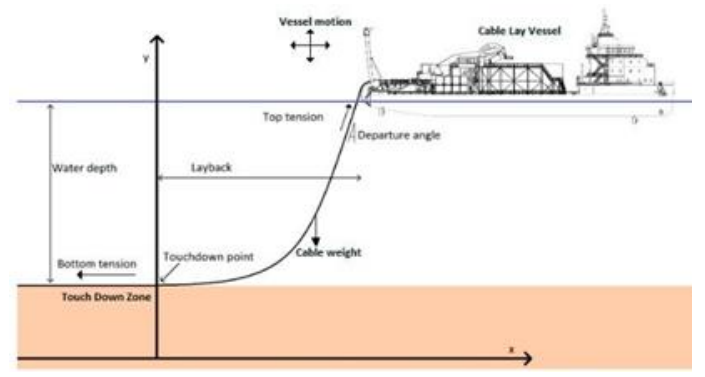

Figure 7 The most influential parameters in an installation operation [8]

\subsection{Submarine cable installation technique}

Submarine power cables can be installed in several methods, new techniques being constantly developed. However, the most common methods for installing submarine cables, depending on how the cable enters the water, are:

- S-lay- this is the most commonly used method. As can be seen in the figure 8 , for the installation of submarine cables a slide is used that guides the cable in the water. It will have a radius greater than or equal to the minimum bend radius (MBR) of the cable to be installed and has no obstructions for accessories or cable-mounted joints. Most offshore wind power cables are installed this way

- S-lay with stinger - this method is similar to the above but is used mainly in deep water and has the role of supporting the cable over a longer length

- J-lay - in this situation the cable will move through a vertical tensioner to keep the value of the MBR within limits, making this method have a good applicability in deeper waters. Another advantage of this method is the increased flexibility in the location of the tensioner, the cable can also be installed through the moonpool, thus reducing the effect of pitching and 


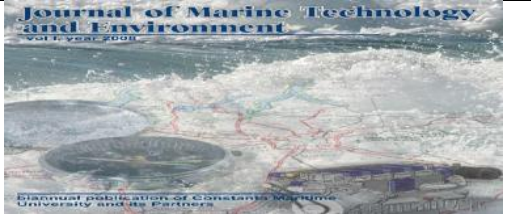

Journal of Marine Technology and Environment Year 2021, Vol. I

rolling the ship on the cable. There are also disadvantages to using this method. These consist in the fact that a minimum depth of water is needed to maintain in the limit the MBR but also the complexity of the installation and operation of the equipment necessary for this operation.
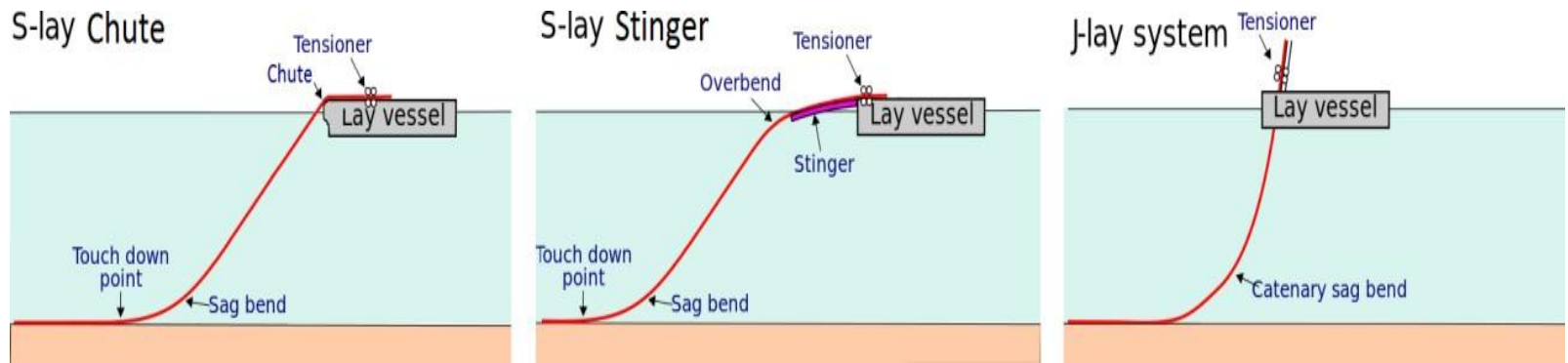

Figure 8 S-lay with Chute, S-lay with Stinger and J-lay [8]

\subsection{Installation of submarine cables in shallow water}

The installation of submarine cables in deep water is done with specialized ships and state-of-the-art equipment. Unfortunately, the installation and repair of submarine cables in shallow water remains a challenge, and it is necessary to develop new concepts to ensure increased efficiency and reduced environmental impact.

As I have seen in several projects in which I have been involved, for the connection to the shore of submarine cables, the installation used techniques, often lead to cable damage and increase the costs of these operations, having a negative impact on the environment. This leads to the decision to use various types of pontoons or barges, with various technical capabilities, with or without their own propulsion. Unfortunately, these installation methods involve the use of a large number of tugs and / or other equipment (fig.9 and fig.10).

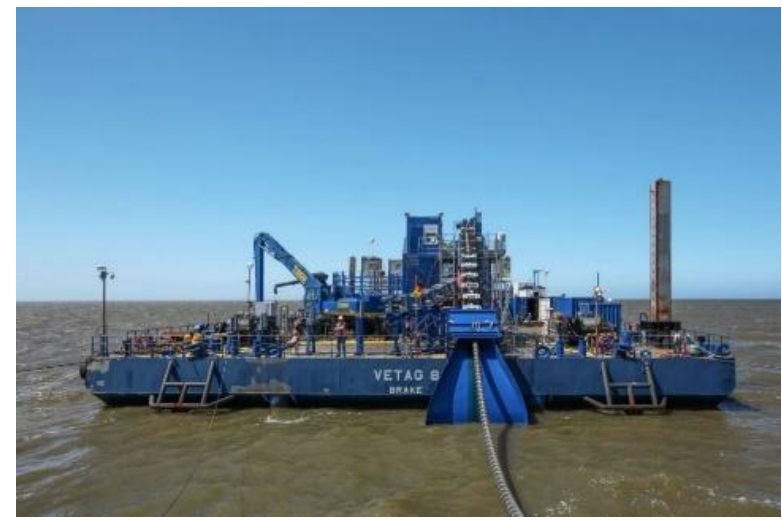

Figure 9 CLV VETAG 8 [9]

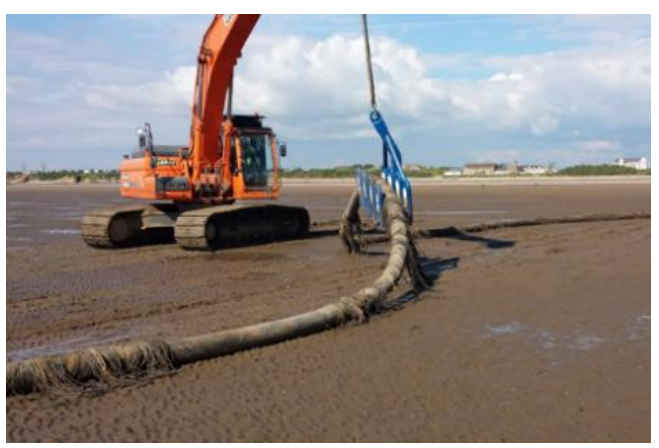

Figure 10 Crawler excavator into intertidal zone [9]

\section{VESSEL MOTION ANALYSIS}

A submarine cable suspended from the chute of an installation vessel will have a catenary shape due to its limited bending stiffness. The catenary shape together with the quadratic drag equations are important sources of non-linear cable response. This chapter explores the character of cable response based on quasi statics and drag. The obtained results will be the basis of the compression limit theory, which will be verified by a numerical analysis.

To study the behaviour and reaction of the cable to the movement of the ship we need a quasi-static analysis from which to obtain the following:

a. Catenary equation - a geometric catenary is the curve that a suspended cable forms under its own weight when it is supported at its ends. In order to simplify the analysis, it will be considered as an ideal situation that the cable material is continuously homogeneous and its bending stiffness and elastic elongation are equal to zero. These ideal conditions are generally acceptable for the installation of submarine cables. Elastic elongation is normally $\varepsilon \mathrm{c}<1 \%$ because high stresses are not desirable and material homogeneity and bending stiffness are also small factors compared to other values that occur during cable installation. [10]. The catenary shape is described by the hyperbolic cosine as in equation (1), its shape is 
In the stationary regime, there must be an equilibrium of forces. The sum of the horizontal forces gives us:

equation based on the force balance with zero bending moment in elements is presented below, the coordinate system in figure 11 is used to obtain the necessary equations that will define the catenary shape.

$$
\operatorname{Tcos}(\varphi)=\mathrm{T}_{0}
$$

$$
y=a \cosh \left(\frac{x}{a}\right)=\frac{a\left(e^{\frac{x}{a}}+e^{-\frac{x}{a}}\right)}{2}
$$

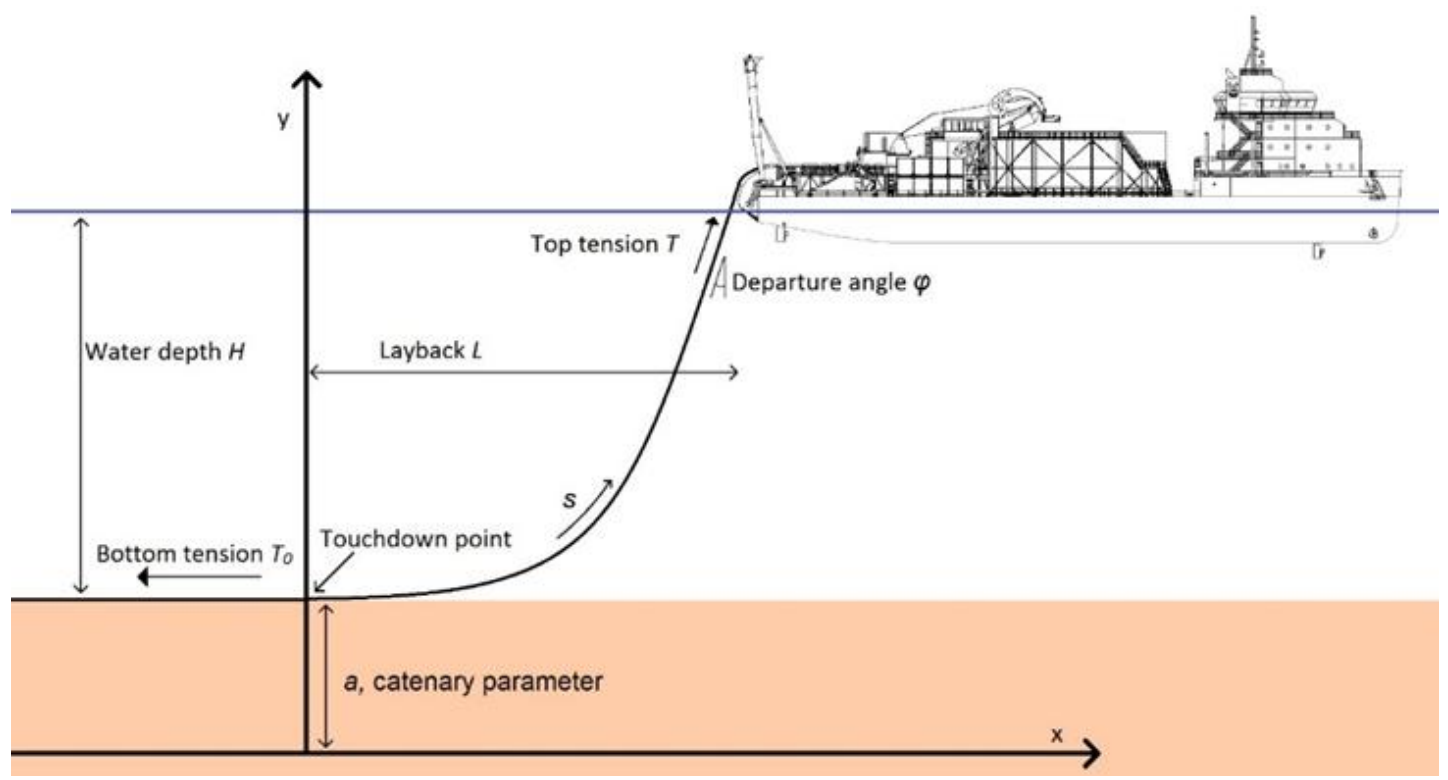

Figure 11 Catenary reference system [8]

The vertical force equilibrium is obtained by substituting $\lambda \mathrm{g}$ as the submerged weight of the cable

$$
T \sin (\varphi)=\lambda g s
$$

Dividing these equations (2\&3) will obtain:

$$
\frac{\mathrm{dy}}{\mathrm{dx}}=\tan (\varphi)=\frac{\lambda \mathrm{gs}}{\mathrm{T}_{0}}
$$

Here "a" is defined as the catenary shape parameter:

$$
\frac{d y}{d x}=\frac{s}{a} \text { were " } a "=\frac{T_{0}}{\lambda g}
$$

The formula for arc length is:

$$
\frac{d s}{d x}=\sqrt{\left(\frac{d y}{d x}\right)^{2}+1}=\frac{\sqrt{a^{2}+s^{2}}}{a}
$$

Which allows:

$$
\frac{d y}{d s}=\frac{d y}{d x} \frac{d x}{d s}=\frac{s}{a} \frac{a}{\sqrt{a^{2}}+s^{2}}=\frac{s}{\sqrt{a^{2}+s^{2}}}
$$

This differential equation can be integrated to:

$$
y=\sqrt{a^{2}+s^{2}}+\beta
$$

For which $\beta$ can be set to 0 by shifting the origin of the $\mathrm{x}$-axis and (6) is integrated with respect to $\mathrm{s}$, to obtain:

$$
x=a \sinh \left(\frac{s}{a}\right)+a
$$

Where again, $\alpha$ can be set to 0 by shifting the $y$ axis, obtaining:

$$
x=a \sinh \left(\frac{s}{a}\right), s=a \sinh \left(\frac{x}{a}\right)
$$

Combining Equation (9) and (10) will be obtain: 


$$
y=\operatorname{acosh}\left(\frac{x}{a}\right)
$$

The catenary is now expressed by the hyperbolic parameter of the cosine and the catenary form "a".

b. Catenary effects - when the chute of the vessel moves in negative surge or heave direction, slackness is obtained in the cable. With enough time, the cable will sink towards the bottom. The tension will decrease, the TDP will move closer to the vessel and the balance of forces is restored.

c. Free fall velocity of the cable - when a segment of cable is horizontally placed in water and let loose, an equilibrium will be formed where the gravitational pull cancels out with the hydrodynamic drag and buoyancy. For this equilibrium, it is assumed that the cable does not rotate and moves through the fluid at right angles to its longitudinal axis.

The vertical force equilibrium demands:

$$
F=F_{d}-F_{g}=0
$$

The drag force is equal to:

$$
F_{d}=\frac{1}{2} \rho_{w} v_{r}^{2} C_{d} D
$$

The gravitational force is equal to:

$$
F_{g}=m_{s} g
$$

Note that the submerged weight is used and therefore the buoyancy is already incorporated.

The normal flow drag coefficient for circular cylinders is dependent on the Reynolds number and surface finish. For the model cable, with a surface roughness of $10^{\wedge}-2$ the drag coefficients profile is shown in figure 12 .

The Reynolds number can be calculated as:

$$
R_{e}=\frac{\rho_{w} v_{r} D}{\mu}
$$

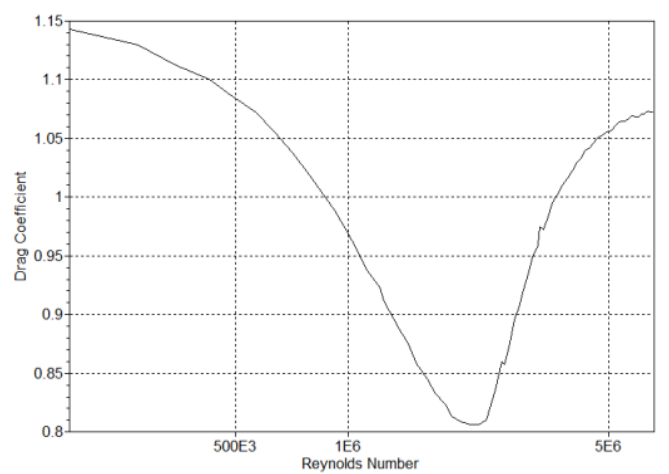

Figure 12 Drag coefficients of model cable

Iteration of the equations: $12-14$ results in a terminal velocity of $1.48 \mathrm{~m} / \mathrm{s}$ at a Reynolds number of $3.9 * 104$. The used parameters are listed in table 1 .

Table 1 Parameters for cable velocity calculation

\begin{tabular}{|c|c|c|c|}
\hline Symbol & Variable & Value & Unit \\
\hline $\mathrm{m}_{\mathrm{s}}$ & submerged weight & 15.87 & $\mathrm{~kg} / \mathrm{m}$ \\
\hline $\mathrm{g}$ & gravity acceleration & 9.81 & $\mathrm{~m} / \mathrm{s}^{2}$ \\
\hline $\mathrm{D}$ & cable diameter & 0.121 & $\mathrm{~m}$ \\
\hline$\rho_{\mathrm{w}}$ & seawater density & 1025 & $\mathrm{~kg} / \mathrm{m}^{3}$ \\
\hline$\mu$ & kinematic viscosity $\left(4^{\circ} \mathrm{C}\right)$ & 0.00467 & $\mathrm{Ns} / \mathrm{m}^{2}$ \\
\hline$C_{d}$ & drag coefficient & 1.143 & - \\
\hline$V_{r}$ & velocity relative to water & 1.48 & $\mathrm{~m} / \mathrm{s}$ \\
\hline
\end{tabular}

From the calculations it is concluded that the cable model cannot fall faster through the water in lateral direction than $1.48 \mathrm{~m} / \mathrm{s}$. Due to the catenary effect a small excitation at the chute will lead to larger lateral excursions along the sag-bend. The same principle works for velocities. A small velocity of the chute in the axial 
direction of the cable, will lead to larger lateral velocities needed to maintain the catenary.

c. Tension loss and compression - when there is a loss of tension in the cable it will sink to the bottom of the sea. Due to the large lateral drag component and limited submerged weight, a significant portion of the cable weight will be affected by the drag. In other words,

when the cable falls through the water, the drag will impose a tension loss along the cable.

If the excitation of the cable is fast enough, the cable is no longer able to follow the catenary shape due to drag forces and all tension can be lost. The cable will move to the bottom with the lateral velocities governed by drag and the axial velocities governed by both inertia and the shape of the falling cable. When the free-falling cable segments hit the bottom with a certain velocity, their momentum will create compression in the cable which is undesirable.

The shape of the falling cable depends on the drag forces, which in turn depend on the velocity and orientation of the cable segments. Therefore, it is impossible to find an analytical expression for the cable under (partial)free fall and compression. Therefore, numerical time domain analysis is required to determine the velocity leading to cable compression.

Effects of TDP movement - as demonstrated above, the displacement of the displacement of the touch down point will be a number of times larger than the excursion of the cable at the chute (TDP). For a relatively low axial velocity, the TDP will have to move at a significant speed to maintain the catenary shape. This may be limited by the transverse wave velocity of the cable.

For a simplified analysis, the transverse wave velocity for a disturbance in a cable without stiffness is represented in equation (16).

$$
v_{t}=\sqrt{\frac{T_{0}}{m}}
$$

\section{ENVIRONMENTAL IMPACT OF SUBMARINE CABLE INSTALLATION}

Submarine cables, like any other external element inserted in the water or on the seabed, can cause disturbances to life and the marine environment. The magnitude of the disturbances refers to the way the cable interacts with the environment: positioned on the seabed or buried in the seabed. In addition to the presence of the cable itself, other influences could be considered, such as induced magnetism, noise, thermal radiation or the chemical and physical interaction between seawater, sediment and the insulation layer of the cable. The cable route can pass through protected areas or sensitive natural environments. The procedure for obtaining permits for the installation of a submarine cable includes an environmental impact assessment (EIA).
A formal EIA usually has at least the following components:

- description of the proposed operation;

- description of the receiving environment (covering all relevant physical, geological factors, biological and anthropogenic / socio-economic); environment;

assessment of potential effects on the

- assessment of mitigation measures necessary to reduce any effects to an environmentally acceptable level;

- assessment of any monitoring measures needed to ensure that the extent of an effect is maintained at an acceptable level.

\subsection{Cables installed on the seabed}

Physical interactions take place on the cables installed on the seabed due to the actions of natural or human factors.

Any interaction of cables with seabed habitats can be assessed by monitoring the biota before and after cable installation [11] or, in the case of installed cables, by comparing the biota in places near and far from a cable [12]. In addition, there are reports of epifauna and epiflora living on cables (Fig.13).

In general, those studies show that the presence of cables has no impact or if it has this impact has minor effects on the resident biota.

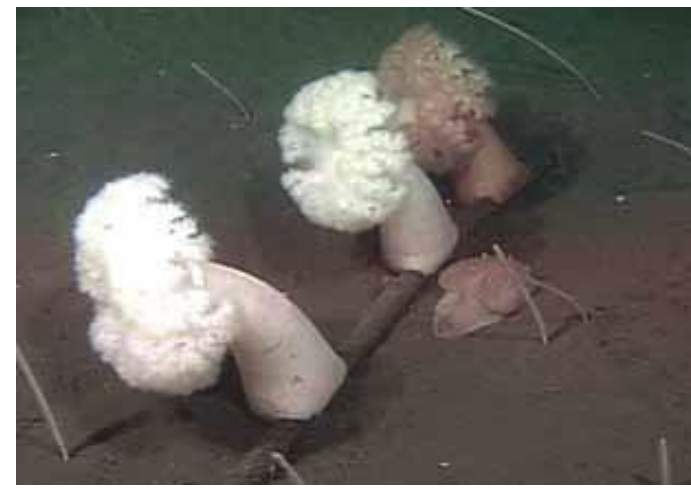

Figure 13 The exposed ATOC/Pioneer Seamount cable with attached anemones [13]

\subsection{Cables buried into the seabed}

Installing cables by burying them at the bottom of the sea can disrupt the marine environment. Compared to other offshore activities, such as bottom trawling, ship anchoring and dredging operations, cable burial operations are limited in time and are a non-repetitive operation, unless a cable needs to be repaired.

Decommissioning and recovery of a buried cable can also lead to disturbances in the marine environment, but again they are of limited and relatively rare 
magnitude, taking into account the projected lifespan of a submarine cable (20-25 years).

\section{CONCLUSIONS}

Ship and cable dynamics are considered uncoupled. The effect that the cable has on the ship's movements is negligible

There is a maximum value of compression that can occur for a certain cable configuration. This depends on the configuration of the catenary and the mass of the cable

With an adequate safety margin, it is possible to estimate the cable compression due to ship movements when installing cables in shallower water.

In terms of the frequency and relatively short duration of submarine cable installation operations, in a small strip of up to $8 \mathrm{~m}$, the disturbances and impact caused by these operations are considered minor as they are preferred over trawling and dredging operations, which are repetitive and more extensive (for example, a single bottom trawl can be tens of meters wide, can sweep substantial areas of the seabed in a single operation and is likely to be repeated over. A single impact, such as cable burial operations, is preferred to continuous, multiple, or recurring impacts.[14]

Uninstallation of cables - as the cables reach the end of their life or due to technological advances, their removal from the seabed may be considered. In the case of a buried cable, removing it can lead to disturbance to marine life. In this context, the cable may support an epifauna that would be lost during a recovery procedure, which is why it may be considered prudent to leave the cable in place to preserve the epifauna.

\section{REFERENCES}

[1] NOAA, 2005. Final Environmental Analysis of Remediation Alternatives for the Pacific Crossing-1 North and East Submarine Fiber-optic Cables in the Olympic Coast National marine Sanctuary. National Oceanographic and Atmospheric Administration.
[2] Mircea A., Philip M., HVDC Submarine Power Cables in the Word, JRC technical report from 2015.

[3] https://www.ngdc.noaa.gov/mgg/image/sedthick9.jpg

[4] https://www.britannica.com/science/continental$\underline{\text { margin }}$

[5] Carter L., Gavey R., Talling P.J. and Liu J.T., 2014, Insights into submarine geohazards from breaks in subsea telecommunication cables, Oceanography.

[6] https://www.aphotomarine.com/geology_sand erosion_wave hub_cable cornwall.html

[7] NOAA, 2006. Hurricanes. National Hurricane Center.

[8] DNV GL. Guideline for installation of rigid and flexible pipelines, umbilicals and subsea power cables Limit state criteria, 2015.

[9] West of Duddon Sands - project , https://westofduddonsands.co.uk/about-the-project

[10] Kurt J. P., Empirical Factors in the Prediction of Helically Armoured Cable Axial Stiffness. s.l.: ASME, 1984.

[11] Andrulewicz E., Napierska D. and Otremba Z., 2003. The environmental effects of the installation and functioning of the submarine SwePol Link HVDC transmission line: A case study of the Polish Marine Area of the Baltic Sea, Journal of Sea Research 49.

[12] Kogan I., Paull C., Kuhnz L., Burton E., Von Thun S., Greene H.G. and Barry J., 2006. ATOC/Pioneer Seamount cable after 8 years on the seafloor: Observations, environmental impact, Continental Shelf Research.

[13] Levings C.D. and McDaniel N.G., 1974. A unique collection of baseline biological data: Benthic invertebrates from an underwater cable across the Strait of Georgia. Fisheries Research Board of Canada, Technical Report 441.

[15] NOAA, 2009. NOAA Report: Four Fish Stocks Declared Fully Rebuilt. 

PUBLISHED SINCE 2008

ISSN:1844-6116

ON LINE SINCE: 2008

PUBLISHED BY: Editura Nautica/ Constanta Maritime University 
ISSN: 1844-6116 